\title{
Stronger Methods of Making Quantum Interactive Proofs Perfectly Complete
}

\author{
Hirotada Kobayashi* $^{*} \quad$ François Le Gall ${ }^{\dagger} \quad$ Harumichi Nishimura $^{\ddagger}$ \\ *Principles of Informatics Research Division \\ National Institute of Informatics \\ Tokyo, Japan \\ ${ }^{\dagger}$ Department of Computer Science \\ Graduate School of Information Science and Technology \\ The University of Tokyo \\ Tokyo, Japan \\ ${ }^{\ddagger}$ Department of Computer Science and Mathematical Informatics \\ Graduate School of Information Science \\ Nagoya University \\ Nagoya, Aichi, Japan
}

\begin{abstract}
This paper presents stronger methods of achieving perfect completeness in quantum interactive proofs. First, it is proved that any problem in QMA has a two-message quantum interactive proof system of perfect completeness with constant soundness error, where the verifier has only to send a constant number of halves of EPR pairs. This in particular implies that the class QMA is necessarily included by the class $\operatorname{QIP}_{1}(2)$ of problems having two-message quantum interactive proofs of perfect completeness, which gives the first nontrivial upper bound for QMA in terms of quantum interactive proofs. It is also proved that any problem having an $m$-message quantum interactive proof system necessarily has an $(m+1)$-message quantum interactive proof system of perfect completeness. This improves the previous result due to Kitaev and Watrous, where the resulting system of perfect completeness requires $m+2$ messages if not using the parallelization result.
\end{abstract}




\section{Introduction}

\subsection{Background and Motivation}

The classical complexity class MA of problems having Merlin-Arthur (MA) proof systems, first introduced by Babai [Bab85], is a natural probabilistic generalization of the class NP. Informally, in a Merlin-Arthur proof system, Arthur, a probabilistic polynomial-time verifier, first receives a message (a witness) from Merlin, an allpowerful but untrustworthy prover, and then checks with high probability the validity of Merlin's claim that the common input is a yes-instance of the problem.

Quantum Merlin-Arthur (QMA) proof systems are a generalization of the Merlin-Arthur proof systems to the quantum setting, whose notion was already discussed at an early stage of quantum computing research in a technical report by Knill [Kni96]. In this setting, Arthur now receives a quantum witness from Merlin and performs polynomial-time quantum computation to check with high probability whether the input is a yes-instance or not. The resulting complexity class is called QMA [Wat00] (originally called BQNP [Kit99, KSV02]), and has been central to the development of quantum complexity theory in that it plays a role similar to that NP plays in classical computation.

The standard way of defining MA and QMA allows two-sided bounded error: each yes-instance may be wrongly rejected with small probability (completeness error), while each no-instance may also be wrongly accepted with small probability (soundness error). If completeness error is zero, that is, any yes-instance is never wrongly rejected, the corresponding system is said to have perfect completeness. The versions of MA and QMA with perfect completeness are denoted by $\mathrm{MA}_{1}$ and $\mathrm{QMA}_{1}$, respectively.

Classically, it is known that any Merlin-Arthur proof system that may have two-sided bounded error can always be modified into another Merlin-Arthur proof system with one-sided bounded error of perfect completeness, i.e., $\mathrm{MA}=\mathrm{MA}_{1}$ holds [ZF87, GZ11]. This is a very nice property in that honest Merlin can always convince Arthur without error by providing a suitable witness for a yes-instance. A natural question to ask is whether the same property holds for quantum Merlin-Arthur proof systems as well, i.e., whether QMA $=\mathrm{QMA}_{1}$ or not. This question still remains unsolved after many years of investigations. Besides its theoretical interest, answering this question by the affirmative would lead to many consequences. In particular, any computational problem complete for the class $\mathrm{QMA}_{1}$, for instance the QUANTUM SATISFIABILITY (QSAT) problems [Bra06], would immediately become complete for the class QMA as well. This would not only lead to a better understanding of QMA but also have potentials to significantly simplify and strengthen a possible quantum version of the celebrated PCP theorem [AS98, $\mathrm{ALM}^{+} 98$ ] that many researchers have been trying to establish [AALV09, AALV11, AE11], partly because one-sided error verifications are much easier to treat, and also because the QSAT problems are more direct quantum analogues of the SAT problems than the LOCAL HAMILTONIAN problems (note that the classical PCP theorem can be viewed as proving the NP-completeness of a special case of the 3SAT problem in which, for every no-instance, at most a constant fraction of clauses are simultaneously satisfiable).

As a barrier to affirmatively answering the QMA versus $\mathrm{QMA}_{1}$ question, Aaronson [Aar09] constructed a quantum oracle relative to which $\mathrm{QMA}_{1}$ is a proper subclass of QMA, which means that a "black-box" proof of $\mathrm{QMA}=\mathrm{QMA}_{1}$ cannot exist. Nevertheless, no classical oracle is known that separates $\mathrm{QMA}_{1}$ from QMA, and the following recent results in some sense step towards an affirmative answer to the question: Nagaj, Wocjan, and Zhang [NWZ09] showed that perfect completeness is achievable for a special case of quantum MerlinArthur proof systems in which some real number related to the maximum acceptance probability of a given system can be exactly expressed with a bit string of polynomial length. More recently, Jordan, Kobayashi, Nagaj, and Nishimura [JKNN12] proved that the equality holds for quantum Merlin-Arthur proof systems of classical witness, that is, $\mathrm{QCMA}=\mathrm{QCMA}_{1}$ (or MQA = $\mathrm{MQA}_{1}$ in a recently-proposed terminology [Wat09a, GSU13]) holds, assuming that the circuit of a verifier is exactly implementable with a gate set in which the Hadamard and any classical reversible transformations are performable without error. In particular, the latter result gives evidence that, if we put some natural assumption on a gate set, the quantum oracle barrier by Aaronson [Aar09] may not be an insur- 
mountable obstacle when seeking the possibility of $\mathrm{QMA}=\mathrm{QMA}_{1}$, as the arguments in Ref. [Aar09] also lead to a quantum oracle that separates $\mathrm{QCMA}_{1}$ from QCMA.

Quantum Merlin-Arthur proof systems may be viewed as a special case of more general quantum interactive proof systems, where the verifier and the prover may exchange messages using many rounds of communications. In their seminal paper, Kitaev and Watrous [KW00] showed that perfect completeness is achievable in quantum interactive proof systems. More precisely, with two additional messages, any quantum interactive proof system that may involve two-sided bounded error can be transformed into another quantum interactive proof system that has one-sided bounded error of perfect completeness. This in particular implies that QMA $\subseteq \mathrm{QIP}_{1}(3)$, where $\mathrm{QIP}_{1}(3)$ is the class of problems having three-message quantum interactive proof systems of perfect completeness. Unfortunately, $\mathrm{QIP}_{1}(3)$ is already so powerful that it includes PSPACE [Wat03] (actually, $\mathrm{QIP}_{1}(3)=\mathrm{QIP}=\mathrm{PSPACE}[\mathrm{KW} 00$, JJUW11], where QIP denotes the class of problems having general quantum interactive proofs). Accordingly, this only gives a weaker result for the upper bound of QMA, as QMA is known to be inside PP [KW00, Wat00, MW05] (in fact, a slightly stronger bound QMA $\subseteq \mathrm{A}_{0} \mathrm{PP}=\mathrm{SBQP}$ is known [Vya03, Kup09]).

\subsection{Our Results and Their Meaning}

This paper presents new general techniques to transform quantum interactive proof systems into those of perfect completeness, which increase the number of messages by just one. Our first result states that any problem in QMA has a two-message quantum interactive proof of perfect completeness.

Theorem 1. $\mathrm{QMA} \subseteq \mathrm{QIP}_{1}(2)$.

Here $\mathrm{QIP}_{1}(2)$ is the class of problems having two-message quantum interactive proof systems of perfect completeness (with negligible soundness error). This gives the first nontrivial upper bound of QMA in terms of quantum interactive proofs, which has no relation known to the existing upper bound $\mathrm{A}_{0} \mathrm{PP}=\mathrm{SBQP}$. Note that the inclusion $\mathrm{QMA} \subseteq \mathrm{QIP}(2)$ is indeed trivial for the two-sided error class $\mathrm{QIP}(2)$ of two-message quantum interactive proofs, but the inclusion here is by the one-sided error class $\mathrm{QIP}_{1}(2)$ and is nontrivial to prove.

In fact, we prove a much stronger result, which arguably steps towards settling the QMA versus $\mathrm{QMA}_{1}$ question. Namely, we show that, to achieve perfect completeness with constant soundness error, the verifier in the two-message quantum interactive proof system has only to send a constant number of halves of EPR pairs to the prover. Or in other words, any problem in QMA has a quantum Merlin-Arthur proof system of perfect completeness with constant soundness error, in which Arthur and Merlin share a constant number of EPR pairs a priori. More formally, let $\mathrm{QMA}^{k \text {-EPR }}(c, s)$ denote the class of problems having quantum Merlin-Arthur proof systems with completeness $c$ and soundness $s$, where Arthur and Merlin initially share $k$ EPR pairs. Then we have the following containment.

Theorem 2. For any constant $s \in(0,1]$, there exists a constant $k \in \mathbb{N}$ such that

$$
\mathrm{QMA} \subseteq \mathrm{QMA}^{k-\mathrm{EPR}}(1, s) .
$$

Theorem 1 is an immediate consequence of Theorem 2 , as one may view quantum Merlin-Arthur proof systems with shared EPR pairs as a special case of two-message quantum interactive proofs where the verifier first generates the EPR pairs and sends halves of them to the prover (and the parallel repetition of two-message quantum interactive proofs works perfectly [KW00]). Theorem 2 nevertheless appears to be much stronger than Theorem 1 since it shows that perfect completeness is achievable with just one additional message of a very restricted form (a constant number of halves of EPR pairs). To see this, let $\mathrm{QMA}^{\text {const-EPR }}$ be the class of problems having quantum Merlin-Arthur proof systems with a constant number of prior shared EPR pairs that may involve two-sided bounded error, and let $\mathrm{QMA}_{1}^{\text {const-EPR }}$ be that of perfect completeness. Then, indeed, the equality $\mathrm{QMA}^{\text {const-EPR }}=\mathrm{QMA}$ immediately follows from the result by Beigi, Shor, and Watrous [BSW11], as any quantum Merlin-Arthur proof 
system with a constant number of prior shared EPR pairs is a special case of two-message quantum interactive proofs with short questions (i.e., two-message quantum interactive proofs with the first message consisting of at most logarithmically many qubits). Therefore, we obtain the following characterization of QMA.

Corollary 3. $\mathrm{QMA}_{1}^{\text {const-EPR }}=\mathrm{QMA}^{\text {const-EPR }}=\mathrm{QMA}$.

This in particular implies that perfect completeness is achievable for the model of quantum Merlin-Arthur proof systems with a constant number of prior shared EPR pairs, a model that has computational power equivalent to QMA. Similar arguments further imply that perfect completeness is achievable even with the models of quantum Merlin-Arthur proof systems with a logarithmic number of prior shared EPR pairs and "short-question" two-message quantum interactive proof systems, as both of these have computational power equivalent to QMA.

The methodology developed in this paper essentially shows that, in order to obtain the inclusion $\mathrm{QMA} \subseteq \mathrm{QMA}_{1}$ (and thus immediately the equality $\mathrm{QMA}=\mathrm{QMA}_{1}$ ), it is sufficient to find a way of eliminating the need for the constant number of shared EPR pairs in our proof system. In fact, as will be clear with our proof structure, the constant number of shared EPR pairs are necessary only for the purpose of forcing a dishonest prover to send a witness that is close to some maximally entangled state of constant dimensions. Hence, some suitable procedure that tests if a given state of constant dimensions is sufficiently entangled or not may replace the shared EPR pairs to affirmatively answer the QMA versus QMA $_{1}$ question (if two-sided error is allowed, such a test is possible with quantum state tomography).

For general quantum interactive proof systems, we further present a method that makes any quantum interactive proof system perfectly complete by increasing the number of messages by just one. This improves the previous result due to Kitaev and Watrous [KW00], whose construction increases the number of messages by two, if not using their parallelization result. More precisely, for the class $\operatorname{QIP}(m)$ of problems having $m$-message quantum interactive proofs that may involve two-sided bounded error, and the class $\mathrm{QIP}_{1}(\mathrm{~m})$ of problems having those of perfect completeness, we show the following.

Theorem 4 (informal statement). For any $m \geq 2$,

$$
\mathrm{QIP}(m) \subseteq \mathrm{QIP}_{1}(m+1)
$$

In fact, if the number of messages in the original system is odd, our transformation does not increase it at all.

Theorem 5 (informal statement). For any odd $m \geq 3$,

$$
\mathrm{QIP}(m) \subseteq \mathrm{QIP}_{1}(m)
$$

While the inclusions of Theorems 4 and 5 can also be obtained by using the parallelization results in Refs. [KW00, KKMV09], our techniques give a new and arguably more direct way of obtaining these results. Our construction actually works well even in the setting of quantum multi-prover interactive proof systems: it transforms any quantum $k$-prover interactive proof system into another quantum $k$-prover interactive proof system of perfect completeness by increasing the number of turns by just one in general, and without increasing it when the number of turns in the original system is odd. This much improves the previous result in Ref. [KKMV09], where the construction increases the number $m$ of turns to $3 m$ (i.e., by a factor of three), again without using their parallelization result. We refer to Theorems 25, 26, 33, and 34 in Section 7for the precise statements of the results. 


\subsection{Organization of This Paper}

Section 2 gives a high-level explanation of how Theorem 2 (i.e, the inclusion $\mathrm{QMA} \subseteq \mathrm{QMA}_{1}^{\text {const-EPR }}$ ) is proved. Section 3 presents an overview of the proof of Theorem 4 (i.e., the inclusion $\left.\mathrm{QIP}(m) \subseteq \mathrm{QIP}_{1}(m+1)\right)$. Section 4 provides basic notions and definitions that are used in this paper. Section 5 rigorously describes and analyzes the basic procedure called REFLECTION PROCEDURE, which is the fundamental technical tool throughout this paper. Section 6 then gives a full proof of Theorem 2. Finally, Section 7 proves the results on general quantum interactive proofs.

\section{Proof Idea of Theorem 2}

The purpose of this section is to give a high-level description of our construction that proves Theorem 2 (the inclusion $\mathrm{QMA} \subseteq \mathrm{QMA}_{1}^{\text {const-EPR }}$ ). We first describe the main idea in Subsection 2.1 and a simple protocol for a very special case. Then we explain in Subsection 2.2 how to make this simple protocol robust against any cheating strategy, by introducing additional tests. Finally, in Subsection 2.3, we present our complete protocol.

\subsection{Underlying Ideas}

For an input $x$, let $V_{x}$ denote the verifier's quantum circuit in the original QMA proof system. The operator $V_{x}$ acts on two quantum registers, one register $\mathrm{A}$ corresponding to the verifier's work space and another register $\mathrm{M}$ corresponding to the space that stores the witness from the prover. Let $p_{x}$ denote the maximum acceptance probability, over all possible witnesses, of the verification procedure. From the definition of the class QMA one can assume that, for every yes-instance $x$ it holds that $p_{x} \geq 1 / 2$, and for every no-instance $x$ it holds that $p_{x} \leq 1 / 3$. As pointed out by Marriott and Watrous [MW05], the maximum acceptance probability $p_{x}$ of $V_{x}$ over all possible witnesses is the maximum eigenvalue of the Hermitian operator

$$
M_{x}=\Pi_{\mathrm{init}} V_{x}^{\dagger} \Pi_{\mathrm{acc}} V_{x} \Pi_{\mathrm{init}},
$$

where $\Pi_{\text {init }}$ is the projection onto the subspace spanned by states in which all the qubits in $A$ are in state $|0\rangle$, and $\Pi_{\text {acc }}$ is the projection onto the space spanned by the accepting states.

Reflection Procedure The basic idea of our protocol is to simulate a procedure that we call REFLECTION PROCEDURE, presented in details in Section 5 Roughly speaking, this procedure is viewed as performing a part of amplitude amplification [Gro96] on the original verification procedure, and is quite similar to the so-called quantum rewinding technique [Wat09b], the underlying idea of which dates back to the strong amplification method for QMA due to Marriott and Watrous [MW05]. Not surprisingly, our REFLECTION PROCEDURE can be analyzed in a way similar to the case of the strong amplification method for QMA due to Marriott and Watrous [MW05]. We refer to Figure 1 for a presentation of this procedure specialized to the case of QMA proof systems (a more general description of the procedure will be given in Figure 3 in Section 5).

The Reflection Procedure has access to the unitary transformation $V_{x}$, receives a quantum state in register $\mathrm{M}$, and has the following property:

1. If $M_{x}$ has an eigenvalue $1 / 2$, then there exists a quantum state in $\mathrm{M}$ such that the procedure accepts with certainty.

2. If $M_{x}$ has no eigenvalue in the interval $\left(\frac{1}{2}-\varepsilon, \frac{1}{2}+\varepsilon\right)$, then for any quantum state in $\mathrm{M}$ given, the procedure rejects with probability at least $4 \varepsilon^{2}$. 


\section{Reflection Procedure}

1. Receive a quantum register M. Prepare $|0\rangle$ in each of the qubits in a quantum register A. Apply $V_{x}$ to the state in $(A, M)$.

2. Perform a phase-flip (i.e., multiply -1 in phase) if the state in $(A, M)$ belongs to the subspace corresponding to the projection $\Pi_{\mathrm{acc}}$.

3. Apply $V_{x}^{\dagger}$ to $(\mathrm{A}, \mathrm{M})$.

4. Reject if the state in $(A, M)$ belongs to the subspace corresponding to $\Pi_{\text {init }}$, and accept otherwise.

Figure 1: The Reflection Procedure (specialized to the case of QMA proof systems; see Figure 3 in Section 5 for the most general version of this procedure).

This procedure would then enable us to transform the original QMA proof system into another QMA proof system with perfect completeness if we had exactly $p_{x}=1 / 2$ for any yes-instance $x$. This nice property on the completeness of course does not necessarily hold in general.

We mention that the REFLECTION PROCEDURE is actually slightly superior to the original quantum rewinding technique (for the purpose of achieving perfect completeness) in that it requires just two applications of $V_{x}$ (more precisely, one application of $V_{x}$ and one application of $V_{x}^{\dagger}$ ), instead of three. This property will be crucial for our analysis since the REFLECTION PROCEDURE will ultimately be applied to a modified version of $V_{x}$ that cannot be implemented directly by the verifier without the help of the prover.

Simple Protocol when $\boldsymbol{p}_{\boldsymbol{x}}$ is Known In general, we only know that $p_{x} \geq 1 / 2$ for a yes-instance. Assume that the verifier can apply the matrix

$$
W_{q}=\left(\begin{array}{cc}
\sqrt{1-q} & \sqrt{q} \\
\sqrt{q} & -\sqrt{1-q}
\end{array}\right)
$$

acting on one qubit, where $q$ is such that $0 \leq q \leq 1$ and $p_{x} q=1 / 2$ (the value of $q$ depends of course on the input $x$ ). Then, by performing in parallel the original verification test (which succeeds with probability $p_{x}$ ) and an additional test that applies $W_{q}$ on a single qubit in the initial state $|0\rangle$ and measures it, we obtain a new verification procedure that accepts the input with probability exactly $p_{x} q=1 / 2$ (where the new condition for acceptance is that the original test accepts and the additional single qubit contains 1). In particular, such a unitary transformation $W_{q}$ always exists for any yes-instance $x$, and thus, this could achieve the perfect completeness if the verifier knew the probability $p_{x} \geq 1 / 2$.

The Hermitian operator corresponding to the case of applying in parallel these two tests can be represented by

$$
M_{x}^{\prime}=\left(\Pi_{\text {init }} \otimes|0\rangle\langle 0|\right)\left(V_{x} \otimes W_{q}\right)^{\dagger}\left(\Pi_{\text {acc }} \otimes|1\rangle\langle 1|\right)\left(V_{x} \otimes W_{q}\right)\left(\Pi_{\text {init }} \otimes|0\rangle\langle 0|\right),
$$

which has $1 / 2$ as an eigenvalue for a yes-instance $x$. Moreover, it can be easily shown that, on a negative instance, the eigenvalues of this Hermitian operator are bounded away from $1 / 2$. Thus, the REFLECTION PROCEDURE applied to the new verification test $V_{x} \otimes W_{q}$ transforms the original system into a perfect completeness system. This protocol of course works only when the verifier can apply $W_{q}$.

Reflection Simulation Test and Distillation Procedure The main problem with the protocol described above is that the verifier does not know in general the probability $p_{x}$, and is then not able to apply $W_{q}$. Informally, our basic idea to overcome this difficulty consists in asking the prover to send, along with the witness $|w\rangle$ of the original 
proof system, the unitary transformation $W_{q}$ to the verifier, where $p_{x} q=1 / 2$. Concretely, this is done by asking the prover to send two copies of the Choi-Jamiotkowski state associated with $W_{q}$, denoted by $\left|J\left(W_{q}\right)\right\rangle$ and defined as follows:

$$
\left|J\left(W_{q}\right)\right\rangle=\left(I \otimes W_{q}\right)\left|\Phi^{+}\right\rangle=\sqrt{1-q}\left|\Phi^{-}\right\rangle+\sqrt{q}\left|\Psi^{+}\right\rangle,
$$

where $\left|\Phi^{+}\right\rangle=\frac{1}{\sqrt{2}}(|00\rangle+|11\rangle),\left|\Phi^{-}\right\rangle=\frac{1}{\sqrt{2}}(|00\rangle-|11\rangle)$ and $\left|\Psi^{+}\right\rangle=\frac{1}{\sqrt{2}}(|01\rangle+|10\rangle)$. By an analysis similar to the case of quantum teleportation, one can see that the state $\left|J\left(W_{q}\right)\right\rangle$ can be used to simulate one application of the unitary transformation $W_{q}$ to any quantum state of a single qubit in a probabilistic manner; the application succeeds with probability $1 / 4$, and we know whether it succeeds or not.

Let us denote by $M$ the register that is expected to contain the witness $|w\rangle$, and by $S_{1}, S_{1}^{\prime}, S_{2}$, and $S_{2}^{\prime}$ the four single-qubit registers that altogether are expected to contain the two copies of the Choi-Jamiołkowski state. On a yes-instance, an (honest) prover will then send the state

$$
|w\rangle_{\mathrm{M}} \otimes\left|J\left(W_{q}\right)\right\rangle_{\left(\mathrm{S}_{1}, \mathrm{~S}_{1}^{\prime}\right)} \otimes\left|J\left(W_{q}\right)\right\rangle_{\left(\mathrm{S}_{2}, \mathrm{~S}_{2}^{\prime}\right)} .
$$

With this state given, the verifier can simulate the desired QMA system with underlying verification procedure $V_{x} \otimes W_{q}$ with success probability $(1 / 4)^{2}=1 / 16$ (note that $W_{q}^{\dagger}=W_{q}$, and thus, one copy of $\left|J\left(W_{q}\right)\right\rangle$ is used to simulate the application of $W_{q}$, and another copy of it is used to simulate the application of $W_{q}^{\dagger}$ ). In case where the simulation fails, the verifier systematically accepts by giving up the simulation to keep perfect completeness. This is the core idea of the procedure REFLECTION SIMULATION TEST described in Subsection 6.1.4, which is a key building block in our proof of Theorem 2

In fact, we incorporate one more technique called Distillation PROCEDURE, which is again based on the analysis of Ref. [MW05], and makes the analysis of our complete protocol significantly easier. In general, one of the main difficulties when analyzing the soundness with the simulation of the REFLECTION PROCEDURE with the associated Hermitian operator $M_{x}^{\prime}$ above is that one has to care about the entanglement between the witness part in $\mathrm{M}$ and the part for the Choi-Jamiołkowski states in $\mathrm{S}_{1}, \mathrm{~S}_{1}^{\prime}, \mathrm{S}_{2}$, and $\mathrm{S}_{2}^{\prime}$. This could make the soundness analysis extremely hard, and in fact, the authors do not even know if the soundness can be proved without using the DISTILlation Procedure. The idea to settle this difficulty is that, instead of directly simulating the REFLECTION PROCEDURE above on a received state (that is expected to be a product state of a witness $|w\rangle$ and two copies of the Choi-Jamiołkowski state), one first performs the Distillation Procedure twice in sequence on the witness part (i.e., M) of the received state to produce a situation where one can perform a much simplified version of the Reflection Procedure that does not even need to receive a witness. This new ReFlection Procedure has a very nice property that it does not significantly change the behavior of the original REFLECTION PROCEDURE, and its associated Hermitian operator acts over a space of just four dimensions and has a much simpler form:

$$
(|0\rangle\langle 0|\otimes| 0\rangle\langle 0|)\left(W_{p} \otimes W_{q}\right)^{\dagger}(|1\rangle\langle 1|\otimes| 1\rangle\langle 1|)\left(W_{p} \otimes W_{q}\right)(|0\rangle\langle 0|\otimes| 0\rangle\langle 0|),
$$

where $p=p_{x}^{2} /\left(2 p_{x}^{2}-2 p_{x}+1\right)$ and $q=1 /(2 p)$ (which is different from the value of $q$ in the previous case with $M_{x}^{\prime}$ ). More precisely, the two applications of the Distillation Procedure (described in Subsection 6.1.1) enable us to generate with high probability two identical copies of the single-qubit state

$$
\left|\chi_{p}\right\rangle=\sqrt{1-p}|0\rangle+\sqrt{p}|1\rangle
$$

from a given witness $|w\rangle$ (and one can know whether the generation of the two copies succeeded or not). The point is that, if the input were a no-instance, and the original soundness were very small, the generated state should be very close to $|0\rangle \otimes|0\rangle$, and could be analyzed as if it were unentangled with other qubits. Note that one can easily transform $\left|\chi_{p}\right\rangle$ into $\left|J\left(W_{p}\right)\right\rangle$, and thus one essentially obtains the desired two copies of the Choi-Jamiołkowski state corresponding to $W_{p}$ after the two applications of the Distillation ProcedurE. 


\subsection{Towards the Actual Protocol}

The main problem of the strategy described in the previous subsection is of course that, on a no-instance, a dishonest prover may not send the prescribed state. Actually, for a dishonest prover who sends a state of the form $|w\rangle \otimes\left|J\left(W_{q}\right)\right\rangle^{\otimes 2}$, then no matter which state $|w\rangle$ and no matter which value $q$ the prover chooses, the soundness can be analyzed with a quite straightforward argument. The real issue lies in the case where a dishonest prover does not send a quantum state of the form $|w\rangle \otimes\left|J\left(W_{q}\right)\right\rangle^{\otimes 2}$, and especially when the state in $\left(\mathrm{S}_{1}, \mathrm{~S}_{1}^{\prime}, \mathrm{S}_{2}, \mathrm{~S}_{2}^{\prime}\right)$ is not a product state of two identical copies of a Choi-Jamiołkowski state.

To force a state in $\left(\mathrm{S}_{1}, \mathrm{~S}_{1}^{\prime}, \mathrm{S}_{2}, \mathrm{~S}_{2}^{\prime}\right)$ to be at least close to a mixture of two-fold products of an identical quantum state (which may be a mixed state), we modify the protocol so that we can use the finite quantum de Finetti theorem [KR05, CKMR07]. For this, the verifier now asks the prover to send not only two copies of $\left|J\left(W_{q}\right)\right\rangle$ but a larger number of copies of it: $\left|J\left(W_{q}\right)\right\rangle^{\otimes N}$ where $N$ is large but still a constant. The expected witness sent by an honest prover is then

$$
|w\rangle_{\mathrm{M}} \otimes\left|J\left(W_{q}\right)\right\rangle_{\left(\mathrm{S}_{1}, \mathrm{~S}_{1}^{\prime}\right)} \otimes \cdots \otimes\left|J\left(W_{q}\right)\right\rangle_{\left(\mathrm{S}_{N}, \mathrm{~S}_{N}^{\prime}\right)} .
$$

The witness state in $\left(\mathrm{M}, \mathrm{S}_{1}, \mathrm{~S}_{1}^{\prime}, \ldots, \mathrm{S}_{N}, \mathrm{~S}_{N}^{\prime}\right)$ sent by a prover in a general case may of course not be of the form above, if the prover is dishonest. After the two applications of the DisTillation ProcedurE with $\mathrm{M}$, the verifier permutes the $N$ pairs of registers $\left(\mathrm{S}_{1}, \mathrm{~S}_{1}^{\prime}\right), \ldots,\left(\mathrm{S}_{N}, \mathrm{~S}_{N}^{\prime}\right)$ uniformly at random. This makes the state in $\left(\mathrm{S}_{1}, \mathrm{~S}_{1}^{\prime}\right), \ldots,\left(\mathrm{S}_{N}, \mathrm{~S}_{N}^{\prime}\right)$ symmetric (i.e., invariant under any permutation of the $N$ pairs of registers $\left(\mathrm{S}_{1}, \mathrm{~S}_{1}^{\prime}\right), \ldots,\left(\mathrm{S}_{N}, \mathrm{~S}_{N}^{\prime}\right)$ ), and thus the quantum de Finetti theorem guarantees that the reduced state in $\left(\mathrm{S}_{1}, \mathrm{~S}_{1}^{\prime}, \mathrm{S}_{2}, \mathrm{~S}_{2}^{\prime}\right)$ of the resulting state after random permutation must be close to some mixture of two-fold product states

$$
\sum_{j} \mu_{j} \xi_{j} \otimes \xi_{j}
$$

Note that each state $\xi_{j}$ may not necessarily be a pure state, and is usually a mixed state. The SWAP TEST, performed additionally to this random permutation, will ensure that every $\xi_{j}$ must be actually close to some pure state. This is nevertheless not enough: we want to ensure that each $\xi_{j}$ is close to some Choi-Jamiołkowski state. To have this desirable property, we now assume that each pair of registers $\left(S_{j}, S_{j}^{\prime}\right)$ initially contains an EPR pair, and that the verifier initially holds the registers $\mathrm{S}_{1}, \ldots, \mathrm{S}_{N}$ and receives only, additionally to $\mathrm{M}$, the registers $\mathrm{S}_{1}^{\prime}, \ldots, \mathrm{S}_{N}^{\prime}$ as witness. This assumption is the only part where we need (a constant number of) shared EPR pairs, and removing it is the last obstacle that prevents us from proving the result $\mathrm{QMA}=\mathrm{QMA}_{1}$. To make use of this assumption, we further device a test called the SPACE RESTRICTION TEST that restricts the Hilbert space corresponding to the registers $\left(S_{1}, S_{1}^{\prime}, S_{2}, S_{2}^{\prime}\right)$ in which the verifier expects to receive the copies of the Choi-Jamiołkowski state. The assumption of a constant number of prior-shared EPR pairs is then tactically used with this SPACE RESTRICTION TEST to finally ensure that each $\xi_{j}$ must be close to some legal Choi-Jamiołkowski state.

\subsection{Final Protocol}

The final protocol of the verifier in a QMA system of perfect completeness with a constant number of shared EPR pairs is given in Figure 2 Actually, Figure 2 presents a slightly simplified exposition of our final protocol; the complete description will appear in Section 6 (see Figure 6 in the proof of Theorem 2).

Let us briefly describe the protocol step by step, focusing on what happens when the prover is honest. At the end of Step 1, i.e., just after receiving a witness from the prover, the state in $\left(\mathrm{M}, \mathrm{S}_{1}, \mathrm{~S}_{1}^{\prime}, \ldots, \mathrm{S}_{N}, \mathrm{~S}_{N}^{\prime}\right)$ is given by

$$
|w\rangle_{\mathrm{M}} \otimes\left|J\left(W_{q}\right)\right\rangle_{\left(\mathrm{S}_{1}, \mathrm{~S}_{1}^{\prime}\right)} \otimes \cdots \otimes\left|J\left(W_{q}\right)\right\rangle_{\left(\mathrm{S}_{N}, \mathrm{~S}_{N}^{\prime}\right)} .
$$

When none of the two executions of the Distillation Procedure fails in Step 2, the state in $\left(\mathrm{R}_{1}, \mathrm{R}_{2}, \mathrm{~S}_{1}, \mathrm{~S}_{1}^{\prime}, \ldots, \mathrm{S}_{N}, \mathrm{~S}_{N}^{\prime}\right)$ becomes

$$
\left|\chi_{p}\right\rangle_{\mathrm{R}_{1}} \otimes\left|\chi_{p}\right\rangle_{\mathrm{R}_{2}} \otimes\left|J\left(W_{q}\right)\right\rangle_{\left(\mathrm{S}_{1}, \mathrm{~S}_{1}^{\prime}\right)} \otimes \cdots \otimes\left|J\left(W_{q}\right)\right\rangle_{\left(\mathrm{S}_{N}, \mathrm{~S}_{N}^{\prime}\right)}
$$


Verifier's QMA Protocol for Achieving Perfect Completeness with $N$ Prior-Shared EPR Pairs (Simplified)

1. Store the particles of the shared $N$ EPR pairs in $\left(\mathrm{S}_{1}, \ldots, \mathrm{S}_{N}\right)$. Receive a quantum witness in registers $\left(\mathrm{M}, \mathrm{S}_{1}^{\prime}, \ldots, \mathrm{S}_{N}^{\prime}\right)$.

2. Execute the Distillation Procedure twice in sequence, both using a state in M. Accept if any of the two executions fails, and continue otherwise, with storing the two generated single-qubit states in $R_{1}$ and $R_{2}$.

3. Permute the $N$ pairs of registers $\left(\mathrm{S}_{1}, \mathrm{~S}_{1}^{\prime}\right), \ldots,\left(\mathrm{S}_{N}, \mathrm{~S}_{N}^{\prime}\right)$ uniformly at random.

4. Perform the Space Restriction Test. That is, test if the state in $\left(\mathrm{S}_{j}, \mathrm{~S}_{j}^{\prime}\right)$ is in the space spanned by $\left\{\left|\Phi^{-}\right\rangle,\left|\Psi^{+}\right\rangle\right\}$, for each $j \in\{1,2\}$. Reject if not so, and continue otherwise.

5. Perform the SwAP TEST between $\left(\mathrm{S}_{1}, \mathrm{~S}_{1}^{\prime}\right)$ and $\left(\mathrm{S}_{2}, \mathrm{~S}_{2}^{\prime}\right)$. Reject if it fails, and continue otherwise.

6. Perform the Reflection Simulation Test with $\left(R_{1}, R_{2}, S_{1}, S_{1}^{\prime}, S_{2}, S_{2}^{\prime}\right)$ as input. Accept if this returns "accept", and reject otherwise.

Figure 2: Slightly simplified description of the verifier's QMA protocol for achieving perfect completeness with $\boldsymbol{N}$ pre-shared EPR pairs. The complete description appears as Figure 6 in Section 6.

at the end of this step. Step 3 just permutes the $N$ pairs of registers $\left(\mathrm{S}_{1}, \mathrm{~S}_{1}^{\prime}\right), \ldots,\left(\mathrm{S}_{N}, \mathrm{~S}_{N}^{\prime}\right)$ uniformly at random, which does not change the state at all. The SPACE RESTRICTION TEST in Step 4 forces each of the two-qubit states in $\left(\mathrm{S}_{1}, \mathrm{~S}_{1}^{\prime}\right)$ and $\left(\mathrm{S}_{2}, \mathrm{~S}_{2}^{\prime}\right)$ to be in the subspace spanned by $\left|\Phi^{-}\right\rangle$and $\left|\Psi^{+}\right\rangle$(as the state must be in this subspace if it is a product of the desirable Choi-Jamiołkowski states), which does not change the state either. Then the SwAP TEST in Step 5 never fails, since the registers $\left(S_{1}, S_{1}^{\prime}\right)$ and $\left(S_{2}, S_{2}^{\prime}\right)$ contain the identical pure state. Finally, Step 6 performs the REFLECTION SIMULATION TEST, which must result in acceptance with certainty, as the value $q$ was chosen appropriately so that the associated Hermitian operator with this REFLECTION Simulation TEST has an eigenvalue exactly $1 / 2$.

Rough Sketch of Soundness Analysis Here we give a very rough sketch of the soundness analysis for a noinstance case. The rigorous analysis can be found in Section 6

Without loss of generality, it is assumed that the original QMA system has soundness exponentially close to 0 . Then, if none of the two executions of the DisTILLATION PROCEDURE fails, whatever witness has been received in Step 1, the state generated in $\left(R_{1}, R_{2}\right)$ after Step 2 must be exponentially close to

$$
\left|\chi_{0}\right\rangle_{R_{1}} \otimes\left|\chi_{0}\right\rangle_{R_{2}}=|0\rangle_{R_{1}} \otimes|0\rangle_{R_{2}}
$$

(and the probability that the DistiLlation Procedure fails is actually exponentially small in this case). This implies that the state in $\left(R_{1}, R_{2}\right)$ is almost unentangled with the state in $\left(S_{1}, S_{1}^{\prime}, \ldots, S_{N}, S_{N}^{\prime}\right)$.

As the random permutation in Step 3 makes the state in $\left(\mathrm{S}_{1}, \mathrm{~S}_{1}^{\prime}, \ldots, \mathrm{S}_{N}, \mathrm{~S}_{N}^{\prime}\right)$ symmetric, from the quantum de Finetti theorem, the reduced state in $\left(R_{1}, R_{2}, S_{1}, S_{1}^{\prime}, S_{2}, S_{2}^{\prime}\right)$ after Step 3 must be close to the state of the form

$$
(|0\rangle\langle 0|)^{\otimes 2} \otimes\left(\sum_{j} \mu_{j} \xi_{j}^{\otimes 2}\right) .
$$

A key property is that the reduced state in $\left(\mathrm{S}_{1}, \mathrm{~S}_{2}\right)$ is exponentially close to the totally mixed state $(I / 2)^{\otimes 2}$, which is guaranteed by the facts that each state in $\mathrm{S}_{j}$ for $j \in\{1, \ldots, N\}$ was originally a half of the shared EPR pair, 
that the two executions of the Distillation PROCEDURE disturbed the state by an amount at most exponentially small, and that the state $(I / 2)^{\otimes N}$ in $\left(\mathrm{S}_{1}, \ldots, \mathrm{S}_{N}\right)$ is invariant under random permutation.

Now one can show that (stated here informally) if the probability of rejection is very small in the SPACE RESTRICTION TEST in Step 4 (otherwise the dishonest prover is caught with some reasonable probability in this Step 4), the state in $\left(R_{1}, R_{2}, S_{1}, S_{1}^{\prime}, S_{2}, S_{2}^{\prime}\right)$ at the end of Step 4 is sufficiently close to a state of the form

$$
(|0\rangle\langle 0|)^{\otimes 2} \otimes\left(\sum_{j} \mu_{j}^{\prime} \xi_{j}^{\otimes 2}\right),
$$

where each $\xi_{j}^{\prime}$ is a mixed state over the Hilbert space spanned by $\left|\Phi^{-}\right\rangle$and $\left|\Psi^{+}\right\rangle$, while the SWAP TEST in Step 5 requires that each $\xi_{j}^{\prime}$ must be close to some pure state (otherwise the dishonest prover is caught with some reasonable probability in this Step 5).

Together with the fact mentioned above that the reduced state in $\left(S_{1}, S_{2}\right)$ was close to the totally mixed state $(I / 2)^{\otimes 2}$ when entering Step 4, these two properties finally ensure that the state in $\left(\mathrm{R}_{1}, \mathrm{R}_{2}, \mathrm{~S}_{1}, \mathrm{~S}_{1}^{\prime}, \mathrm{S}_{2}, \mathrm{~S}_{2}^{\prime}\right)$ at the end of Step 5 must be sufficiently close to a state of the form

$$
(|0\rangle\langle 0|)^{\otimes 2} \otimes\left[\sum_{j} \mu_{j}^{\prime \prime}\left(\left|J\left(W_{a_{j}}^{ \pm}\right)\right\rangle\left\langle J\left(W_{a_{j}}^{ \pm}\right)\right|\right)^{\otimes 2}\right],
$$

where each $W_{a_{j}}^{ \pm}$is equal to either $W_{a_{j}}$ or $Z W_{a_{j}} Z$, with $Z=\left(\begin{array}{cc}1 & 0 \\ 0 & -1\end{array}\right)$. Notice that this is a mixture of desired states and their slightly different variants.

For each state of the form

$$
|0\rangle^{\otimes 2} \otimes\left|J\left(W_{a_{j}}^{ \pm}\right)\right\rangle^{\otimes 2}=\left|\chi_{0}\right\rangle^{\otimes 2} \otimes\left|J\left(W_{a_{j}}^{ \pm}\right)\right\rangle^{\otimes 2}
$$

however, we can easily show that the REFLECTION Simulation TeSt in Step 6 rejects with sufficiently large probability (shown to be exactly $1 / 16$ ) irrelevant to the value $a_{j}$, and thus, the verifier can reject with probability close to $1 / 16$ even when the verification procedure reaches Step 6 with very high probability.

\section{Proof Idea of Theorem 4}

This section gives an overview of the proof of Theorem 4 (more precisely, of the formal statement of this result, Theorem 25), which proves the inclusion $\mathrm{QIP}(m) \subseteq \mathrm{QIP}_{1}(m+1)$, for each $m \geq 2$. For simplicity, here we assume that the number $m$ of messages is odd (the case with even number of messages can be proved with essentially the same argument), and completeness and soundness are $2 / 3$ and $1 / 3$, respectively, in the original quantum interactive proof system.

The basic idea is again to simulate the REFLECTION PROCEDURE associated with the original $m$-message quantum interactive proof system.

Fix an input $x$ and the transformations of the prover $P$ on $x$ in the original $m$-message quantum interactive proof system. This time, we consider that the register $M$ in the REFLECTION PROCEDURE described in Figure 1 contains all the qubits the prover $P$ can access in the original system (i.e., all the private qubits of the prover and all the message qubits that are used for communications). We further consider that the register A contains all the private qubits of the verifier in the original system. Now, if we replace $V_{x}$ in Figure 1 by the unitary transformation $U$ derived from the original quantum interactive proof system when the verifier communicates with $P$ on input $x$, the Reflection Procedure described in Figure 1 can be viewed as first applying $U$ by performing a forward simulation of the communications with $P$, then applying a phase-flip with respect to the accepting states, and further applying $U^{\dagger}$ by performing a backward simulation of the communications with $P$ to confirm if the entire state does not go back to a legal initial state. 
Hence, if there is a strategy for a prover that can convince the verifier with probability exactly $1 / 2$ in the original system, then this specific REFLECTION PROCEDURE with such a prover must result in acceptance with certainty, from the property of the REFLECTION PROCEDURE. Fortunately, if the number $m$ of messages is at least two, it is not hard for an all powerful prover to arbitrarily decrease the accepting probability, and thus, this essentially achieves the perfect completeness when the input is a yes-instance. On the other hand, for any no-instance, no prover can convince the verifier with probability more than $1 / 3$. This implies that the above specific REFLECTION PROCEDURE must result in rejection with some constant probability (actually with probability at least $1 / 9$ ), again from the property of the REFLECTION PROCEDURE. Therefore, this basically establishes a quantum interactive proof system of perfect completeness, as desired.

There are two problems in this construction. One is that a dishonest prover may not be so cooperative that a backward simulation forms $U^{\dagger}$ as required (i.e., a prover may behave during the backward simulation differently from the inverse of what he/she behaved during the forward simulation). The other is that the number of messages increases from $m$ to $2 m-1$, and thus, it is less communication-efficient than the existing construction of achieving perfect completeness in quantum interactive proofs due to Kitaev and Watrous [KW00].

Modified Reflection Procedure Both of the two problems mentioned above originate from the fact that the REFLECTION PROCEDURE involves one application of $U$ and one application of $U^{\dagger}$. Now we modify the procedure so that it involves one application of $U^{\dagger}$ only (and no application of $U$ is required), which simultaneously settles both of the two problems.

To do this, at the beginning, one expects to receive a state just after Step 1 of the REFLECTION PROCEDURE, and then performs on this state two tests, called REFLECTION TEST and INVERTIBILITY TEST, respectively, with equal probability without revealing which test the prover is undergoing. In the REFLECTION TEST, one simply performs Steps 2-4 of the REFLECTION Procedure (i.e., one first applies the appropriate phase-flip and then applies $U^{\dagger}$ ) to finish the simulation of it. In the INVERTIBILITY TEST, one apply just $U^{\dagger}$ without performing the phase-flip and checks if the entire state does go back to a legal initial state of the original REFLECTION PROCEDURE. We call the resulting procedure the Modified Reflection PROCEDURE, a precise description of which will be given in Subsection 7.1. The idea of making use of the INVERTIBILITY TEST originally appeared in Ref. [KKMV09] when achieving perfect completeness in quantum multi-prover interactive proofs, but the test was used only after the forward simulation of the protocol in their original construction, and was not for the purpose of reducing the number of messages.

As is clear from the construction above, the MODIFIED REFLECTION PROCEDURE requires only one application of $U^{\dagger}$ as desired. Thus, the quantum interactive proof system that simulates this MODIFIED REFLECTION PROCEDURE involves only $m$ messages as required (for an even $m$, it involves $m+1$ messages, as the original system starts with a turn for a verifier, while the verifier in the constructed system needs to receive a witness before his/her first turn). Moreover, for any yes-instance, the honest prover clearly has only to cooperate with the verifier to perform the backward simulation of the original REFLECTION PROCEDURE and can convince the verifier with certainty. On the other hand, for any no-instance, the original REFLECTION PROCEDURE would have rejected with high probability, if the proper $U^{\dagger}$ had been performed. Thus, if the backward simulation in the MODIFIED REFLECTION PROCEDURE were properly performed, the REFLECTION TEST of it could reject with high probability as it properly simulates the original REFLECTION PROCEDURE. In contrast, if the backward simulation were not proper in the MOdified REFLECTION PROCEDURE, then the INVERTIBILITY TEST of it would result in rejection with high probability, as it essentially forces the prover to perform a proper backward simulation of the original REFLECTION PROCEDURE. Indeed, as will be proved in Subsection 7.1 if one starts with a REFLECTION PROCEDURE that rejects with probability at least $\varepsilon$ for every possible witness, the resulting MODIFIED REFLECTION PROCEDURE rejects with probability at least $\varepsilon / 4$ no matter which witness is received (the proof of Proposition 30 essentially proves this). Hence, the soundness can be shown as well in the ModifiEd ReFLECTION ProcedurE. 


\section{Preliminaries}

Throughout this paper, let $\mathbb{N}$ and $\mathbb{Z}^{+}$denote the sets of positive and nonnegative integers, respectively, and let $\Sigma=\{0,1\}$ denote the binary alphabet set. A function $f: \mathbb{Z}^{+} \rightarrow \mathbb{N}$ is polynomially bounded if there exists a polynomial-time deterministic Turing machine that outputs $1^{f(n)}$ on input $1^{n}$. A function $f: \mathbb{Z}^{+} \rightarrow[0,1]$ is negligible if, for every polynomially bounded function $g: \mathbb{Z}^{+} \rightarrow \mathbb{N}$, it holds that $f(n)<1 / g(n)$ for all but finitely many values of $n$.

Quantum Fundamentals We assume the reader is familiar with the quantum formalism, including pure and mixed quantum states, density operators, measurements, trace norm, fidelity, as well as the quantum circuit model (see Refs. [NC00, KSV02], for instance). Here we summarize some notations and properties that are used in this paper.

For each $k \in \mathbb{N}$, let $\mathbb{C}\left(\Sigma^{k}\right)$ denote the $2^{k}$-dimensional complex Hilbert space whose standard basis vectors are indexed by the elements in $\Sigma^{k}$. In this paper, all Hilbert spaces are complex and have dimension a power of two. For a Hilbert space $\mathcal{H}$, let $I_{\mathcal{H}}$ denote the identity operator over $\mathcal{H}$, and let $\mathbf{D}(\mathcal{H})$ be the set of density operators over $\mathcal{H}$. For a quantum register $\mathrm{R}$, let $|0\rangle_{\mathrm{R}}$ denote the state in which all the qubits in $\mathrm{R}$ are in state $|0\rangle$. As usual, denote the two single-qubit states in $\mathbb{C}(\Sigma)$ that form the Hadamard basis by

$$
|+\rangle=\frac{1}{\sqrt{2}}(|0\rangle+|1\rangle), \quad|-\rangle=\frac{1}{\sqrt{2}}(|0\rangle-|1\rangle),
$$

and the four two-qubit states in $\mathbb{C}\left(\Sigma^{2}\right)$ that form the Bell basis by

$$
\begin{array}{ll}
\left|\Phi^{+}\right\rangle=\frac{1}{\sqrt{2}}(|00\rangle+|11\rangle), \quad\left|\Phi^{-}\right\rangle=\frac{1}{\sqrt{2}}(|00\rangle-|11\rangle), \\
\left|\Psi^{+}\right\rangle=\frac{1}{\sqrt{2}}(|01\rangle+|10\rangle), \quad\left|\Psi^{-}\right\rangle=\frac{1}{\sqrt{2}}(|01\rangle-|10\rangle),
\end{array}
$$

respectively. Let

$$
H=\frac{1}{\sqrt{2}}\left(\begin{array}{cc}
1 & 1 \\
1 & -1
\end{array}\right), \quad X=\left(\begin{array}{ll}
0 & 1 \\
1 & 0
\end{array}\right), \quad Z=\left(\begin{array}{cc}
1 & 0 \\
0 & -1
\end{array}\right)
$$

denote the Hadamard and Pauli operators. For convenience, we may identify a unitary operator with the unitary transformation it induces. In particular, for a unitary operator $U$, the induced unitary transformation is also denoted by $U$.

For a linear operator $A$, the trace norm of $A$ is defined by

$$
\|A\|_{\operatorname{tr}}=\operatorname{tr} \sqrt{A^{\dagger} A}
$$

For two quantum states $\rho$ and $\sigma$, the trace distance between them is defined by

$$
D(\rho, \sigma)=\frac{1}{2}\|\rho-\sigma\|_{\mathrm{tr}},
$$

and the fidelity between them is defined by

$$
F(\rho, \sigma)=\operatorname{tr} \sqrt{\sqrt{\rho} \sigma \sqrt{\rho}} .
$$

A special case of the trace distance is the statistical difference between two probability distributions $\mu$ and $\nu$, which is defined by

$$
\mathrm{SD}(\mu, \nu)=D(\mu, \nu)
$$

by viewing probability distributions as special cases of quantum states with diagonal density operators. We will use the following important properties of the trace distance and fidelity. 
Lemma 6. Let $\mu_{\rho}$ and $\mu_{\sigma}$ be the probability distributions derived from two quantum states $\rho$ and $\sigma$, respectively, by performing an arbitrary identical measurement. Then,

$$
\mathrm{SD}\left(\mu_{\rho}, \mu_{\sigma}\right) \leq D(\rho, \sigma) .
$$

Lemma 7 ([SR02, NS03]). For any quantum states $\rho, \sigma$, and $\xi$,

$$
F(\rho, \sigma)^{2}+F(\sigma, \xi)^{2} \leq 1+F(\rho, \xi) .
$$

For any unitary transformation $U$ acting over the two-dimensional Hilbert space $\mathcal{H}=\mathbb{C}(\Sigma)$ (i.e., the singlequbit space), the Choi-Jamiotkowski state of $U$ is the two-qubit state in $\mathcal{H} \otimes \mathcal{H}=\mathbb{C}\left(\Sigma^{2}\right)$ defined by

$$
|J(U)\rangle=(I \otimes U)\left|\Phi^{+}\right\rangle .
$$

In fact, the Choi-Jamiołkowski state can be defined for any admissible (and not limited to unitary) transformation and any finite-dimensional Hilbert space, using the Choi-Jamiołkowski representation [Jam72, Cho75], but which is unnecessary in this paper.

The Finite Quantum de Finetti Theorem For $N \in \mathbb{N}$ and quantum registers $\mathrm{Q}_{1}, \ldots, \mathrm{Q}_{N}$, each consisting of $k$ qubits, an $N$-partite quantum state $\rho$ in $\left(Q_{1}, \ldots, Q_{N}\right)$ is said to be symmetric if $\rho$ is invariant under any permutation over the registers $\mathrm{Q}_{1}, \ldots, \mathrm{Q}_{N}$.

The finite quantum de Finetti theorem [KR05, CKMR07] provides a very useful property that the reduced $m$-partite state of any $N$-partite symmetric state when tracing out the last $N-m$ subsystems must be close to a mixture of $m$-fold product states. This paper uses the following bound proved in Ref. [CKMR07].

Theorem 8 (Finite quantum de Finetti theorem). For $N, k \in \mathbb{N}$, let $\mathrm{Q}_{1}, \ldots, \mathrm{Q}_{N}$ be quantum registers each consisting of $k$ qubits, and let $\rho$ be an $N$-partite symmetric state in $\left(Q_{1}, \ldots, Q_{N}\right)$. For any $m \in \mathbb{N}$ satisfying $m<N$ and the m-partite reduced state $\rho^{(m)}$ of $\rho$ in $\left(Q_{1}, \ldots, Q_{m}\right)$, there exist $C \in \mathbb{N}$, a set $\left\{\xi_{j}\right\}_{j \in\{1, \ldots, C\}}$ of $k$-qubit states, and an associated probability distribution $\left\{\mu_{j}\right\}_{j \in\{1, \ldots, C\}}$ such that

$$
D\left(\rho^{(m)}, \sum_{j=1}^{C} \mu_{j} \xi_{j}^{\otimes m}\right) \leq \frac{2^{2 k+1} m}{N} .
$$

Polynomial-Time Uniformly Generated Families of Quantum Circuits Following conventions, we define quantum Merlin-Arthur proof systems in terms of quantum circuits. In particular, we use the following notion of polynomial-time uniformly generated families of quantum circuits.

A family $\left\{Q_{x}\right\}$ of quantum circuits is polynomial-time uniformly generated if there exists a deterministic procedure that, on every input $x$, outputs a description of $Q_{x}$ and runs in time polynomial in $|x|$. It is assumed that the circuits in such a family are composed of gates in some reasonable, universal, finite set of quantum gates. Furthermore, it is assumed that the number of gates in any circuit is not more than the length of the description of that circuit. Therefore $Q_{x}$ must have size polynomial in $|x|$. For convenience, we may identify a circuit $Q_{x}$ with the unitary operator it induces.

Throughout this paper, we assume a gate set with which the Hadamard and any classical reversible transformations can be exactly implemented. Note that this assumption is satisfied by many standard gate sets such as the Shor basis [Sho96] consisting of the Hadamard, controlled- $i$-phase-shift, and Toffoli gates, and the gate set consisting of 
the Hadamard, Toffoli, and NOT gates [Shi02, Aha03]. Moreover, as the Hadamard transformation in some sense can be viewed as a quantum analogue of the classical operation of flipping a fair coin, our assumption would be the most natural quantum correspondence to the tacit classical assumption in randomized complexity theory that fair coins and perfect logical gates are available. Hence we believe that our condition is very reasonable and not restrictive. Note that, with a gate set satisfying this assumption, any transformation corresponding to a Clifford group operator is exactly implementable. In particular, the controlled-phase-flip transformation $Z$ can be exactly realized by using an ancilla qubit prepared in state $|-\rangle=\frac{1}{\sqrt{2}}(|0\rangle-|1\rangle$ ) (by applying a NOT and an Hadamard in sequence to $|0\rangle$ ) and performing a CNOT with this ancilla as the target.

Since non-unitary and unitary quantum circuits are equivalent in computational power [AKN98], it is sufficient to treat only unitary quantum circuits, which justifies the above definition. Nevertheless, for readability, most procedures in this paper will be described using intermediate projective measurements and unitary operations conditioned on the outcome of the measurements. All of these intermediate measurements can be deferred to the end of the procedure by a standard technique so that the procedure becomes implementable with a unitary circuit.

Quantum Interactive Proof Systems Now we review the model of quantum interactive proof systems.

A quantum interactive proof system is a communication model between two players called a quantum verifier $V$ and a quantum prover $P$, both of whom receive a common input $x \in \Sigma^{*}$. Fix the input $x$. Let $\mathrm{V}$ and $\mathrm{P}$ be quantum registers corresponding to the private spaces of $V$ and $P$, respectively, and let $\mathrm{M}$ be a quantum register corresponding to the message space that is used to exchange messages between $V$ and $P$. One of the qubits in $\mathrm{V}$, which is private to $V$, is designated as the output qubit. At the beginning, all the qubits in $\mathrm{V}$ and $\mathrm{M}$ are initialized to state $|0\rangle$, while the quantum state in $\mathrm{P}$ can be arbitrarily prepared by $P$. Then $V$ and $P$ together run a protocol that consists of alternating turns of the verifier and of the prover. The first turn is for the verifier if the total number of turns is even, and it is for the prover otherwise, whereas the last turn is always for the prover. At each turn of the verifier, $V$ applies some unitary transformation implementable with a polynomial-size quantum circuit to the state in $(\mathrm{V}, \mathrm{M})$, and then sends the register $\mathrm{M}$ to $P$. At each turn of the prover, $P$ applies some unitary transformation to the state in $(\mathrm{P}, \mathrm{M})$, and then sends $\mathrm{M}$ to $V$. After the last turn, the verifier $V$ further applies some unitary transformation implementable with a polynomial-size quantum circuit to the state in $(V, M)$, and then measures the output qubit in the standard basis. $V$ accepts if this measurement results in $|1\rangle$ and rejects otherwise.

Formally, for any function $m: \mathbb{Z}^{+} \rightarrow \mathbb{N}$ that is polynomially bounded, an m-message polynomial-time quantum verifier is a polynomial-time computable mapping $V: \Sigma^{*} \rightarrow \Sigma^{*}$. For each input $x \in \Sigma^{*}, V(x)$ is interpreted as describing a series $\left\{V_{x, j}\right\}_{j \in\{1, \ldots, \Gamma(m(|x|)+1) / 2\rceil\}}$ of quantum circuits acting over the same number of qubits as well as a partition of the qubits on which these circuits act into registers $\mathrm{V}$ and $\mathrm{M}$, where $\left\{V_{x, j}\right\}$ is a polynomialtime uniformly generated family of quantum circuits explained before (in particular, every circuit $V_{x, j}$ is composed of gates in some reasonable, universal, finite set of quantum gates). For any polynomially bounded function $m: \mathbb{Z}^{+} \rightarrow \mathbb{N}$, an m-message quantum prover is a mapping $P$ that simply maps an input binary string $x \in \Sigma^{*}$ to a series $\left\{P_{x, j}\right\}_{j \in\{1, \ldots,\lfloor(m(|x|)+1) / 2\rfloor\}}$ of unitary transformations as well as a partition of the qubits on which these unitary transformations act into registers $\mathrm{M}$ and $\mathrm{P}$. It is always assumed that $V$ and $P$ are compatible (i.e., the register $\mathrm{M}$ is common for $V$ and $P$ ) when they are associated with the same quantum interactive proof system.

Given an input $x$, an $m$-message polynomial-time quantum verifier $V$, and an $m$-message quantum prover $P$, let $Q_{x}$ be the unitary transformation induced from $V$ and $P$, acting over the space corresponding to $(\mathrm{V}, \mathrm{M}, \mathrm{P})$ :

$$
Q_{x}=\left(V_{x,(m(|x|)+1) / 2} \otimes I_{\mathcal{P}}\right)\left(I_{\mathcal{V}} \otimes P_{x,(m(|x|)+1) / 2}\right) \cdots\left(V_{x, 1} \otimes I_{\mathcal{P}}\right)\left(I_{\mathcal{V}} \otimes P_{x, 1}\right)
$$

if $m(|x|)$ is odd, while

$$
Q_{x}=\left(V_{x,(m(|x|) / 2)+1} \otimes I_{\mathcal{P}}\right)\left(I_{\mathcal{V}} \otimes P_{x, m(|x|) / 2}\right)\left(V_{x, m(|x|) / 2} \otimes I_{\mathcal{P}}\right) \cdots\left(I_{\mathcal{V}} \otimes P_{x, 1}\right)\left(V_{x, 1} \otimes I_{\mathcal{P}}\right)
$$

if $m(|x|)$ is even, where $\mathcal{V}$ and $\mathcal{P}$ are the Hilbert spaces corresponding to $\mathrm{V}$ and $\mathrm{P}$, respectively. When communicating with the prover $P$ who prepares the initial state $\rho \in \mathbf{D}(\mathcal{P})$, the verifier $V$ accepts the input $x$ if the measurement 
of the designated output qubit in $\mathrm{V}$ in the standard basis results in $|1\rangle$ at the end of the protocol after having applied the unitary transformation $Q_{x}$ to the initial state $|0\rangle\left\langle\left. 0\right|_{(\mathrm{V}, \mathrm{M})} \otimes \rho\right.$ in $(\mathrm{V}, \mathrm{M}, \mathrm{P})$.

Formally, the class $\mathrm{QIP}(m, c, s)$ of problems having $m$-message quantum interactive proof systems with completeness $c$ and soundness $s$ is defined as follows. For generality, throughout this paper, we use promise problems [ESY84] rather than languages when defining complexity classes.

Definition 9. Given a polynomially bounded function $m: \mathbb{Z}^{+} \rightarrow \mathbb{N}$ and functions $c, s: \mathbb{Z}^{+} \rightarrow[0,1]$ satisfying $c>s$, a promise problem $A=\left(A_{\text {yes }}, A_{\text {no }}\right)$ is in $\mathrm{QIP}(m, c, s)$ iff there exists an $m$-message polynomial-time quantum verifier $V$ such that, for every input $x$ :

(Completeness) if $x \in A_{\text {yes }}$, there exist an $m$-message quantum prover $P$ and the initial state $\rho_{x}$ of $P$ that make $V$ accept $x$ with probability at least $c(|x|)$,

(Soundness) if $x \in A_{\text {no }}$, for any $m$-message quantum prover $P^{\prime}$ and any initial state $\rho_{x}^{\prime}$ of $P^{\prime}$ prepared, $V$ accepts $x$ with probability at most $s(|x|)$.

The class $\mathrm{QIP}(m)$ of problems having $m$-message quantum interactive proof systems is defined as follows.

Definition 10. Given a polynomially bounded function $m: \mathbb{Z}^{+} \rightarrow \mathbb{N}$, a promise problem $A=\left(A_{\text {yes }}, A_{\text {no }}\right)$ is in $\mathrm{QIP}(m)$ iff $A$ is in $\operatorname{QIP}(m, 1-\varepsilon, \varepsilon)$ for some negligible function $\varepsilon: \mathbb{Z}^{+} \rightarrow[0,1]$.

Similarly, the class $\mathrm{QIP}_{1}(m)$ of problems having $m$-message quantum interactive proof systems of perfect completeness is defined as follows.

Definition 11. Given a polynomially bounded function $m: \mathbb{Z}^{+} \rightarrow \mathbb{N}$, a promise problem $A=\left(A_{\text {yes }}, A_{\text {no }}\right)$ is in $\mathrm{QIP}_{1}(m)$ iff $A$ is in $\operatorname{QIP}(m, 1, \varepsilon)$ for some negligible function $\varepsilon: \mathbb{Z}^{+} \rightarrow[0,1]$.

Finally, as quantum Merlin-Arthur proof systems are nothing but one-message quantum interactive proof systems, the classes QMA and $\mathrm{QMA}_{1}$ of problems having quantum Merlin-Arthur proof systems and those of perfect completeness are simply defined as follows, respectively.

Definition 12. A promise problem $A=\left(A_{\text {yes }}, A_{\text {no }}\right)$ is in QMA iff $A$ is in $\mathrm{QIP}(1,1-\varepsilon, \varepsilon)$ for some negligible function $\varepsilon: \mathbb{Z}^{+} \rightarrow[0,1]$.

Definition 13. A promise problem $A=\left(A_{\text {yes }}, A_{\text {no }}\right)$ is in $\mathrm{QMA}_{1}$ iff $A$ is in $\mathrm{QIP}(1,1, \varepsilon)$ for some negligible function $\varepsilon: \mathbb{Z}^{+} \rightarrow[0,1]$.

Quantum Merlin-Arthur Proof Systems with Shared EPR Pairs We further introduce another variant of quantum Merlin-Arthur proof systems in which Arthur and Merlin initially share some copies of the EPR pair $\left|\Phi^{+}\right\rangle$. If Arthur and Merlin are allowed to share $k$ EPR pairs initially, the resulting systems are called quantum MerlinArthur proof systems with $k$ shared EPR pairs, or $k$-EPR QMA proof systems in short. Notice that this model is actually equivalent to a special case of two-message quantum interactive proof systems in which the first transformation of a verifier is just to create $k$ copies of the EPR pairs (and $k$ halves of these EPR pairs are sent to a prover as the first message).

Formally, the class QMA ${ }^{k-E P R}(c, s)$ of problems having quantum Merlin-Arthur proof systems with $k$ shared EPR pairs with completeness $c$ and soundness $s$ is defined as follows.

Definition 14. Given a polynomially bounded function $k: \mathbb{Z}^{+} \rightarrow \mathbb{N}$ and functions $c, s: \mathbb{Z}^{+} \rightarrow[0,1]$ satisfying $c>s$, a promise problem $A=\left(A_{\text {yes }}, A_{\text {no }}\right)$ is in $\mathrm{QMA}^{k-\mathrm{EPR}}(c, s)$ iff $A$ has a two-message quantum interactive proof system with completeness $c$ and soundness $s$ in which, for every input $x$, the first transformation of the associated quantum verifier is just to create $k(|x|)$ copies of EPR pairs and the first message from the verifier consists only of the $k(|x|)$ halves of these EPR pairs. 
We further define the class $\mathrm{QMA}^{\text {const-EPR }}$ of problems having quantum Merlin-Arthur proof systems with a constant number of shared EPR pairs with constant gap between completeness and soundness and the class $\mathrm{QMA}_{1}^{\text {const-EPR }}$ of problems having those of perfect completeness with constant soundness error as follows.

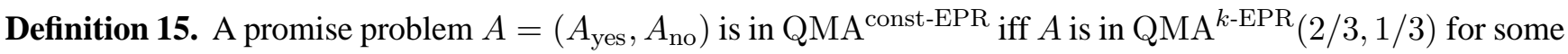
constant $k \in \mathbb{N}$.

Definition 16. A promise problem $A=\left(A_{\text {yes }}, A_{\text {no }}\right)$ is in $\mathrm{QMA}_{1}^{\text {const-EPR }}$ iff $A$ is in $\mathrm{QMA}^{k \text {-EPR }}(1,1 / 2)$ for some constant $k \in \mathbb{N}$.

Remark. Definitions 15 and 16 are equivalent to the seemingly most conservative defini-

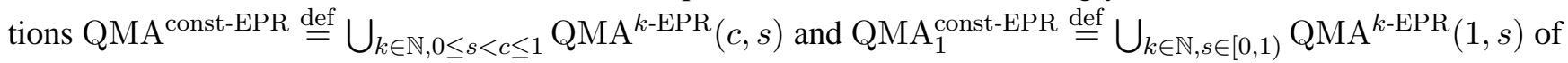
these classes, for repeating the associated system with each of these classes constant times can achieve arbitrarily large constant gap between completeness and soundness (in the two-sided error case, one first achieves sufficiently large completeness via a parallel repetition followed by a threshold value computation, and then achieves desirably small soundness via another parallel repetition of the obtained large-completeness system, without decreasing the completeness too much).

\section{Reflection Procedure}

We start with presenting a very simple base procedure, which we call the REFLECTION PROCEDURE, that forms a very base of our protocols to be constructed - basically, our protocols aim to simulate this base procedure with several suitable modifications.

Let $\mathcal{H}$ be some Hilbert space, and consider two decompositions of $\mathcal{H}$ into $\mathcal{X}_{0} \oplus \mathcal{X}_{1}$ and $\mathcal{Y}_{0} \oplus \mathcal{Y}_{1}$ for subspaces $\mathcal{X}_{0}, \mathcal{X}_{1}, \mathcal{Y}_{0}$, and $\mathcal{Y}_{1}$ of $\mathcal{H}$. Let $\Delta_{j}$ be the projection over $\mathcal{H}$ onto the subspace $\mathcal{X}_{j}$ and let $\Pi_{j}$ be that onto $\mathcal{Y}_{j}$, for each $j \in\{0,1\}$.

Let $U$ be some unitary transformation acting over $\mathcal{H}$, and let $M$ be the Hermitian operator over $\mathcal{H}$ defined by

$$
M=\Delta_{0} U^{\dagger} \Pi_{0} U \Delta_{0}
$$

Suppose that $M$ has an eigenvalue $\lambda>0$ and consider the eigenstate (i.e., the normalized eigenvector) $\left|\phi_{0}\right\rangle$ corresponding to $\lambda$. Then, $M\left|\phi_{0}\right\rangle=\lambda\left|\phi_{0}\right\rangle$, and thus,

$$
\Delta_{0}\left|\phi_{0}\right\rangle=\frac{1}{\lambda} \Delta_{0} M\left|\phi_{0}\right\rangle=\frac{1}{\lambda} M\left|\phi_{0}\right\rangle=\left|\phi_{0}\right\rangle
$$

Define the four states $\left|\psi_{0}\right\rangle,\left|\psi_{1}\right\rangle,\left|\xi_{0}\right\rangle$, and $\left|\xi_{1}\right\rangle$ in $\mathcal{H}$ as follows:

$$
\left|\psi_{0}\right\rangle=\frac{\Pi_{0} U\left|\phi_{0}\right\rangle}{\| \Pi_{0} U\left|\phi_{0}\right\rangle \|}, \quad\left|\psi_{1}\right\rangle=\frac{\Pi_{1} U\left|\phi_{0}\right\rangle}{\| \Pi_{1} U\left|\phi_{0}\right\rangle \|}, \quad\left|\xi_{0}\right\rangle=\frac{\Delta_{0} U^{\dagger}\left|\psi_{0}\right\rangle}{\| \Delta_{0} U^{\dagger}\left|\psi_{0}\right\rangle \|}, \quad\left|\xi_{1}\right\rangle=\frac{\Delta_{1} U^{\dagger}\left|\psi_{0}\right\rangle}{\| \Delta_{1} U^{\dagger}\left|\psi_{0}\right\rangle \|} .
$$

Then, $\| \Pi_{0} U\left|\phi_{0}\right\rangle\|=\| \Pi_{0} U \Delta_{0}\left|\phi_{0}\right\rangle \|=\sqrt{\left\langle\phi_{0}|M| \phi_{0}\right\rangle}=\sqrt{\lambda}$, and thus, $\| \Pi_{1} U\left|\phi_{0}\right\rangle \|=\sqrt{1-\lambda}$. It follows that

$$
\| \Delta_{0} U^{\dagger}\left|\psi_{0}\right\rangle\left\|=\frac{1}{\sqrt{\lambda}}\right\| \Delta_{0} U^{\dagger} \Pi_{0} U\left|\phi_{0}\right\rangle\left\|=\frac{1}{\sqrt{\lambda}}\right\| \Delta_{0} U^{\dagger} \Pi_{0} U \Delta_{0}\left|\phi_{0}\right\rangle\left\|=\frac{1}{\sqrt{\lambda}}\right\| M\left|\phi_{0}\right\rangle \|=\sqrt{\lambda}
$$

and thus, $\| \Delta_{1} U^{\dagger}\left|\psi_{0}\right\rangle \|=\sqrt{1-\lambda}$. Hence,

$$
\left|\xi_{0}\right\rangle=\frac{1}{\sqrt{\lambda}} \Delta_{0} U^{\dagger}\left|\psi_{0}\right\rangle=\frac{1}{\lambda} \Delta_{0} U^{\dagger} \Pi_{0} U\left|\phi_{0}\right\rangle=\frac{1}{\lambda} \Delta_{0} U^{\dagger} \Pi_{0} U \Delta_{0}\left|\phi_{0}\right\rangle=\frac{1}{\lambda} M\left|\phi_{0}\right\rangle=\left|\phi_{0}\right\rangle .
$$




\section{Reflection Procedure}

1. Receive a quantum register $Q$. Reject if the state in $Q$ does not belong to the subspace corresponding to the projection $\Delta_{0}$, and otherwise apply $U$ to $\mathrm{Q}$.

2. Perform a phase-flip (i.e., multiply -1 in phase) if the state in $Q$ belongs to the subspace corresponding to the projection $\Pi_{0}$.

3. Apply $U^{\dagger}$ to $Q$.

4. Reject if the state in $\mathrm{Q}$ belongs to the subspace corresponding to $\Delta_{0}$, and accept otherwise.

Figure 3: The Reflection Procedure.

This implies that

$$
U^{\dagger}\left|\psi_{0}\right\rangle=\sqrt{\lambda}\left|\xi_{0}\right\rangle+\sqrt{1-\lambda}\left|\xi_{1}\right\rangle, \quad U^{\dagger}\left|\psi_{1}\right\rangle=\sqrt{1-\lambda}\left|\xi_{0}\right\rangle-\sqrt{\lambda}\left|\xi_{1}\right\rangle
$$

which was the crucial property analyzed by Marriott and Watrous [MW05] to develop their space-efficient QMA amplification technique.

It follows that

$$
U^{\dagger}\left(-\Pi_{0}+\Pi_{1}\right) U\left|\phi_{0}\right\rangle=U^{\dagger}\left(-\sqrt{\lambda}\left|\psi_{0}\right\rangle+\sqrt{1-\lambda}\left|\psi_{1}\right\rangle\right)=(1-2 \lambda)\left|\xi_{0}\right\rangle-2 \sqrt{\lambda(1-\lambda)}\left|\xi_{1}\right\rangle,
$$

and thus, when $M$ has an eigenvalue $1 / 2$, the corresponding eigenstate (which is necessarily in $\mathcal{X}_{0}$ ) must be transformed into a state in $\mathcal{X}_{1}$ after the following process: one first applies $U$ to $\left|\phi_{0}\right\rangle$, next flips the phase of states in $\mathcal{Y}_{0}$ (i.e., applies the unitary transformation $-\Pi_{0}+\Pi_{1}$ ), and then applies $U^{\dagger}$. This property can be used to test if $M$ has an eigenvalue $1 / 2$, which is summarized in Figure 3.

Proposition 17. Suppose that the Hermitian operator $M=\Delta_{0} U^{\dagger} \Pi_{0} U \Delta_{0}$ has an eigenvalue $1 / 2$. Then there exists a quantum state given in Step 1 of the REFLECTION PROCEDURE such that the procedure results in acceptance with certainty.

Proof. Consider the case where the eigenstate of $M$ with its corresponding eigenvalue $1 / 2$ is received in $Q$ in Step 1. Then the claim is immediate from the argument above.

Proposition 18. For any $\varepsilon \in\left(0, \frac{1}{2}\right]$, suppose that none of the eigenvalues of the Hermitian operator $M=\Delta_{0} U^{\dagger} \Pi_{0} U \Delta_{0}$ is in the interval $\left(\frac{1}{2}-\varepsilon, \frac{1}{2}+\varepsilon\right)$. Then, for any quantum state given in Step 1 of the REFLECTION PROCEDURE, the procedure results in rejection with probability at least $4 \varepsilon^{2}$.

Proof. Let $|\psi\rangle$ be any state received in $\mathrm{Q}$ in Step 1. Without loss of generality, one can assume that $|\psi\rangle$ is in $\mathcal{X}_{0}$ (as otherwise either rejected in Step 1 or projected onto a state in $\mathcal{X}_{0}$ ).

For the Hilbert space $\mathcal{H}$, there always exists an orthonormal basis such that all the basis states of it are eigenstates of $M$, and thus, the state $|\psi\rangle$ can be necessarily written as $|\psi\rangle=\sum_{j=1}^{d} \alpha_{j}\left|\phi_{j}\right\rangle$ for $d=\operatorname{dim} \mathcal{X}_{0} \leq \operatorname{dim} \mathcal{H}$, where each $\left|\phi_{j}\right\rangle$ is an eigenstate of $M$ in $\mathcal{X}_{0}$ and $\sum_{j=1}^{d}\left|\alpha_{j}\right|^{2}=1$.

From the analysis above, every eigenstate $\left|\phi_{j}\right\rangle$ of $M$ in $\mathcal{X}_{0}$ with corresponding eigenvalue $\lambda_{j}>0$ must satisfy that

$$
\Delta_{0} U^{\dagger}\left(-\Pi_{0}+\Pi_{1}\right) U\left|\phi_{j}\right\rangle=\left(1-2 \lambda_{j}\right)\left|\phi_{j}\right\rangle .
$$

On the other hand, for every eigenstate $\left|\phi_{j}\right\rangle$ of $M$ in $\mathcal{X}_{0}$ with corresponding eigenvalue $\lambda_{j}=0$, it holds that $\| \Pi_{0} U\left|\phi_{j}\right\rangle\|=\| \Pi_{0} U \Delta_{0}\left|\phi_{j}\right\rangle \|=\sqrt{\left\langle\phi_{j}|M| \phi_{j}\right\rangle}=0$. This implies $\Pi_{1} U\left|\phi_{j}\right\rangle=U\left|\phi_{j}\right\rangle$, and thus,

$$
\Delta_{0} U^{\dagger}\left(-\Pi_{0}+\Pi_{1}\right) U\left|\phi_{j}\right\rangle=\Delta_{0}\left|\phi_{j}\right\rangle=\left|\phi_{j}\right\rangle=\left(1-2 \lambda_{j}\right)\left|\phi_{j}\right\rangle
$$


Therefore,

$$
\Delta_{0} U^{\dagger}\left(-\Pi_{0}+\Pi_{1}\right) U|\psi\rangle=\sum_{j=1}^{d} \alpha_{j}\left(1-2 \lambda_{j}\right)\left|\phi_{j}\right\rangle,
$$

and thus, the probability of rejection is at least $\sum_{j=1}^{d}\left|\alpha_{j}\right|^{2}\left(1-2 \lambda_{j}\right)^{2} \geq 4 \varepsilon^{2} \sum_{j=1}^{d}\left|\alpha_{j}\right|^{2}=4 \varepsilon^{2}$, as claimed.

\section{$6 \quad \mathrm{QMA} \subseteq \mathrm{QMA}_{1}^{\text {const-EPR }} \subseteq \mathrm{QIP}_{1}(2)$}

The goal of this section is to prove Theorem 2 In Subsection 6.1 we first describe building blocks, before presenting the proof in Subsection 6.2.

\subsection{Building Blocks}

\subsubsection{Encoding Accepting Probability in Phase}

Let $V$ be the verifier of a certain QMA system. Consider the quantum circuit $V_{x}$ of $V$ when the input is $x$, which acts over a pair of two registers $\mathrm{A}$ of $v(|x|)$ qubits and $\mathrm{M}$ of $m(|x|)$ qubits, for some polynomially bounded functions $v, m: \mathbb{Z}^{+} \rightarrow \mathbb{N}$. The circuit $V_{x}$ expects to receive a quantum witness of $m(|x|)$ qubits in register $\mathrm{M}$, and uses the $v(|x|)$ qubits in $\mathrm{A}$ as its work qubits. The Hilbert spaces associated with $\mathrm{A}$ and $\mathrm{M}$ are denoted by $\mathcal{A}$ and $\mathcal{M}$, respectively.

For an input $x$, let $p_{x}$ be the maximum acceptance probability of the verifier $V$ in this QMA system. Then, as pointed out by Marriott and Watrous [MW05], $p_{x}$ corresponds to the maximum eigenvalue of the Hermitian operator

$$
M_{x}=\Pi_{\text {init }} V_{x}^{\dagger} \Pi_{\text {acc }} V_{x} \Pi_{\text {init }},
$$

where $\Pi_{\text {init }}$ is the projection onto the subspace spanned by states in which all the qubits in $A$ are in state $|0\rangle$, and $\Pi_{\text {acc }}$ is that onto the subspace spanned by accepting states of this QMA system. Let $\left|w_{x}\right\rangle$ be the eigenstate (i.e., eigenvector) of $M_{x}$ corresponding to the eigenvalue $p_{x}$. A crucial analysis of Ref. [MW05] (which essentially follows from the arguments in Section [5) is that

$$
\begin{aligned}
& \Pi_{\text {init }} V_{x}^{\dagger} \Pi_{\text {acc }} V_{x}\left(|0\rangle_{\mathrm{A}} \otimes\left|w_{x}\right\rangle_{\mathrm{M}}\right)=p_{x}|0\rangle_{\mathrm{A}} \otimes\left|w_{x}\right\rangle_{\mathrm{M}}, \\
& \Pi_{\text {init }} V_{x}^{\dagger} \Pi_{\mathrm{rej}} V_{x}\left(|0\rangle_{\mathrm{A}} \otimes\left|w_{x}\right\rangle_{\mathrm{M}}\right)=\left(1-p_{x}\right)|0\rangle_{\mathrm{A}} \otimes\left|w_{x}\right\rangle_{\mathrm{M}},
\end{aligned}
$$

where $\Pi_{\text {rej }}=I_{\mathcal{A} \otimes \mathcal{M}}-\Pi_{\text {acc }}$ is the projection onto the subspace spanned by rejecting states of this QMA system.

Let $p=p_{x}^{2} /\left(2 p_{x}^{2}-2 p_{x}+1\right)$. Using the property explained above, if one copy of $\left|w_{x}\right\rangle$ is given, one can generate with high probability the state

$$
\left|\chi_{p}\right\rangle=\frac{1}{\sqrt{2 p_{x}^{2}-2 p_{x}+1}}\left[\left(1-p_{x}\right)|0\rangle+p_{x}|1\rangle\right]
$$

as follows. One uses a single-qubit register $\mathrm{R}$ in addition to $\mathrm{A}$ and $\mathrm{M}$, where one sets $\left|w_{x}\right\rangle$ in $\mathrm{M}$, and initializes all the qubits in $A$ and $R$ to state $|0\rangle$. First, one performs a forward simulation of the original system over $A$ and $M$ (i.e., applies $V_{x}$ to $(\mathrm{A}, \mathrm{M})$ ), and flips the qubit in $\mathrm{R}$ if the content of $(\mathrm{A}, \mathrm{M})$ corresponds to an accepting state of the original system (i.e., applies the unitary transformation $X \otimes \Pi_{\mathrm{acc}}+I \otimes \Pi_{\mathrm{rej}}$ to $(\mathrm{R}, \mathrm{A}, \mathrm{M})$ ). One then performs a backward simulation of the original system over $A$ and $M$ (i.e., applies $V_{x}^{\dagger}$ to $(A, M)$ ). Now one measures all the qubits in $A$ in the computational basis. If no $|1\rangle$ is measured (i.e., if the state is projected with respect to $\Pi_{\text {init }}$, which happens with probability $\left.2 p_{x}^{2}-2 p_{x}+1\right)$, the unnormalized state in the system must be

$$
|0\rangle_{\mathrm{R}} \otimes\left(1-p_{x}\right)|0\rangle_{\mathrm{A}} \otimes\left|w_{x}\right\rangle_{\mathrm{M}}+|1\rangle_{\mathrm{R}} \otimes p_{x}|0\rangle_{\mathrm{A}} \otimes\left|w_{x}\right\rangle_{\mathrm{M}}=\left[\left(1-p_{x}\right)|0\rangle+p_{x}|1\rangle\right]_{\mathrm{R}} \otimes|0\rangle_{\mathrm{A}} \otimes\left|w_{x}\right\rangle_{\mathrm{M}},
$$

and thus, the desired state is successfully generated in R. We call this procedure the DistiLlation ProcedurE, which is summarized in Figure 4 


\section{Distillation Procedure}

Input: a single-qubit register $\mathrm{R}$, a $v(|x|)$-qubit register $\mathrm{A}$, and an $m(|x|)$-qubit register $\mathrm{M}$.

Output: a single-qubit register $\mathrm{R}$ or a symbol $\perp$.

1. Apply $V_{x}$ to $(\mathrm{A}, \mathrm{M})$.

2. Flip the qubit in $\mathrm{R}$ if the content of $(\mathrm{A}, \mathrm{M})$ corresponds to an accepting state of the original system.

3. Apply $V_{x}^{\dagger}$ to $(\mathrm{A}, \mathrm{M})$.

4. Measures all the qubits in $A$ in the computational basis. If any of these measurements result in $|1\rangle$, output $\perp$, otherwise output $R$.

Figure 4: The Distillation PROcedure.

\subsubsection{Multiplicatively Adjusting Accepting Probabilities}

For a real number $a \in[0,1]$, let $W_{a}$ be the unitary transformation defined by

$$
W_{a}=\left(\begin{array}{cc}
\sqrt{1-a} & \sqrt{a} \\
\sqrt{a} & -\sqrt{1-a}
\end{array}\right)
$$

Given a unitary transformation $W_{p}$ for some real number $p \in\left[\frac{1}{2}, 1\right]$, we construct another unitary transformation $U$ and an appropriate projection operator $\Pi_{0}$ acting over two qubits so that the probability $\| \Pi_{0} U|00\rangle \|^{2}$ exactly equals $1 / 2$.

Suppose that one can apply another unitary transformation $W_{q}$, for some real number $q \in[0,1]$, and define the unitary transformation $U$ and projection operator $\Pi_{0}$ by

$$
U=W_{p} \otimes W_{q}, \quad \Pi_{0}=|11\rangle\langle 11| .
$$

Then, clearly, $\| \Pi_{0} U|00\rangle \|^{2}=p q$, and thus, this probability equals $1 / 2$ if and only if $p q=1 / 2$. This in particular implies that there exists a real number $q \in[0,1]$ that achieves the adjusted accepting probability exactly $1 / 2$ when $p \geq 1 / 2$, but no $q \in[0,1]$ can make it exactly equal to $1 / 2$ when $p<1 / 2$.

\subsubsection{Simulating Unitaries with Choi-Jamiołkowski States}

In this subsection, we consider the case where the aforementioned unitary transformation $W_{a}$ itself is not available, but only the copies of its Choi-Jamiołkowski state $\left|J\left(W_{a}\right)\right\rangle=\left(I \otimes W_{a}\right)\left|\Phi^{+}\right\rangle$are available.

Note that one copy of the Choi-Jamiołkowski state $\left|J\left(W_{a}\right)\right\rangle$ can be used to simulate one application of $W_{a}$ (the simulation succeeds with probability $1 / 4)$. More precisely, the simulation of $W_{a}$ is done as follows. Suppose one wants to apply $W_{a}$ to the qubit in some single-qubit register $\mathrm{R}_{1}$, while the state $\left|J\left(W_{a}\right)\right\rangle$ is available in $\left(\mathrm{R}_{2}, \mathrm{R}_{2}^{\prime}\right)$, for some single-qubit registers $R_{2}$ and $R_{2}^{\prime}$. Then one measures the state in $\left(R_{1}, R_{2}\right)$ in the Bell basis. If this results in $\left|\Phi^{+}\right\rangle$, the application of $W_{a}$ succeeds, and the desired state is available in the register $\mathrm{R}_{2}^{\prime}$ (which can be verified via an argument similar to the analysis of seminal quantum teleportation).

Actually, when one wants to apply $W_{a}$ to the specific state $|0\rangle$, there is a more efficient way than simulation just explained above. A key observation is that, for any real number $a \in[0,1]$, the unitary transformation $W_{a}$ in 
the last subsection can be written as

$$
W_{a}=\left(\begin{array}{cc}
\sqrt{1-a} & \sqrt{a} \\
\sqrt{a} & -\sqrt{1-a}
\end{array}\right)=\sqrt{1-a} Z+\sqrt{a} X
$$

and thus, the state $\left|\chi_{a}\right\rangle$ is given by

$$
\left|\chi_{a}\right\rangle=W_{a}|0\rangle=\sqrt{1-a}|0\rangle+\sqrt{a}|1\rangle,
$$

while the Choi-Jamiołkowski state of $W_{a}$ is given by

$$
\left|J\left(W_{a}\right)\right\rangle=\sqrt{1-a}|J(Z)\rangle+\sqrt{a}|J(X)\rangle=\sqrt{1-a}\left|\Phi^{-}\right\rangle+\sqrt{a}\left|\Psi^{+}\right\rangle .
$$

Hence, given one copy of the Choi-Jamiołkowski state $\left|J\left(W_{a}\right)\right\rangle$, one can easily generate the state $\left|\chi_{a}\right\rangle=W_{a}|0\rangle$ in the first qubit by applying the following unitary transformation $T$ to $\left|J\left(W_{a}\right)\right\rangle$ :

$$
T:\left|\Phi^{-}\right\rangle \mapsto|00\rangle, \quad\left|\Psi^{-}\right\rangle \mapsto|01\rangle, \quad\left|\Psi^{+}\right\rangle \mapsto|10\rangle, \quad\left|\Phi^{+}\right\rangle \mapsto|11\rangle
$$

(note that this $T$ can be realized by first applying the CNOT transformation using the first qubit as the control, then applying the Hadamard transformation $H$ and the NOT transformation $X$ in this order to the first qubit, and finally applying CNOT again using the first qubit as the control).

\subsubsection{Simulating the Reflection Procedure with Choi-Jamiołkowski States}

Now we consider simulating the ReFlection Procedure with given two copies of $\left|\chi_{p}\right\rangle=W_{p}|0\rangle$ and two copies of a Choi-Jamiołkowski state $\left|J\left(W_{q}\right)\right\rangle$, where $p$ and $q$ are real numbers in $[0,1]$. The procedure basically follows the Reflection Procedure with taking the register Q to be a two-qubit register, the initial state $\left|\phi_{0}\right\rangle$ to be $|00\rangle$, the projection $\Delta_{0}$ to be $|00\rangle\langle 00|$, and the underlying unitary $U$ and projection $\Pi_{0}$ to be $W_{p} \otimes W_{q}$ and $|11\rangle\langle 11|$, as defined in Subsection 6.1.2 Thus, to precisely perform the REFLECTION PROCEDURE in Figure 3 in this setting, we need to apply each of $W_{p}=W_{p}^{\dagger}$ and $W_{q}=W_{q}^{\dagger}$ twice. Fortunately, each of the first applications of $W_{p}$ and $W_{q}$ is to the $|0\rangle$ state, and thus, one may simply replace these applications by just using a given copy of $\left|\chi_{p}\right\rangle$ and generating $\left|\chi_{q}\right\rangle$ from a copy of $\left|J\left(W_{q}\right)\right\rangle$, respectively. The second applications of these unitaries can be probabilistically simulated by using the Choi-Jamiołkowski states $\left|J\left(W_{p}\right)\right\rangle$ and $\left|J\left(W_{q}\right)\right\rangle$, where one creates $\left|J\left(W_{p}\right)\right\rangle$ from a copy of $\left|\chi_{p}\right\rangle$. This leads to the procedure called REFLECTION Simulation Test described in Figure 5

Now we analyze the properties of this simulation.

Proposition 19. The REFLECTION SIMULATION TEST accepts with certainty if the state in the input regis$\operatorname{ter}\left(\mathrm{R}_{1}, \mathrm{R}_{2}, \mathrm{~S}_{1}, \mathrm{~S}_{1}^{\prime}, \mathrm{S}_{2}, \mathrm{~S}_{2}^{\prime}\right)$ is $\left|\chi_{p}\right\rangle^{\otimes 2} \otimes\left|J\left(W_{q}\right)\right\rangle^{\otimes 2}$ for some real numbers $p, q \in[0,1]$ satisfying $p q=1 / 2$.

Proof. The claim is almost obvious. With $\left|\chi_{p}\right\rangle$ in $\mathrm{R}_{1}$ and $\left|J\left(W_{q}\right)\right\rangle$ in $\left(\mathrm{S}_{1}, \mathrm{~S}_{1}^{\prime}\right)$ for such $p$ and $q$, Step 1 in the REFLECTION Simulation Test creates the state

$$
U|00\rangle=(\sqrt{1-p}|0\rangle+\sqrt{p}|1\rangle)_{\mathrm{R}_{1}} \otimes(\sqrt{1-q}|0\rangle+\sqrt{q}|1\rangle)_{\mathrm{S}_{1}}
$$

in $\left(R_{1}, S_{1}\right)$, since the application of $T$ generates the state $\left|\chi_{q}\right\rangle$ in $S_{1}$. As the application of $T^{\dagger}$ in Step 3 generates the Choi-Jamiołkowski state $\left|J\left(W_{p}\right)\right\rangle$ in $\left(\mathrm{R}_{2}, \mathrm{R}_{2}^{\prime}\right)$, one succeeds in Step 3 with probability $(1 / 4)^{2}=1 / 16$ in applying both of $W_{p}^{\dagger}=W_{p}$ and $W_{q}^{\dagger}=W_{q}$, which successfully simulates $U^{\dagger}$ with generating the desired state in $\left(\mathrm{R}_{2}^{\prime}, \mathrm{S}_{2}^{\prime}\right)$. Hence, the simulation of the REFLECTION PROCEDURE succeeds with probability $1 / 16$, in which case the test necessarily results in acceptance as in the analysis in Section 5, since $\left(|00\rangle\left\langle 00\left|U^{\dagger} \Pi_{0} U\right| 00\right\rangle\langle 00|\right)|00\rangle=\| \Pi_{0} U|00\rangle \|^{2}|00\rangle=\frac{1}{2}|00\rangle$. On the other hand, if any of measurements in Step 3 fails in measuring $\left|\Phi^{+}\right\rangle$, the test just stops and accepts with giving up. Therefore, the test must result in acceptance with certainty. 


\section{REFLECTION Simulation TEST}

Input: single-qubit registers $\mathrm{R}_{1}, \mathrm{R}_{2}, \mathrm{~S}_{1}, \mathrm{~S}_{1}^{\prime}, \mathrm{S}_{2}$, and $\mathrm{S}_{2}^{\prime}$.

Output: "accept" or "reject".

1. Receive six single-qubit registers $R_{1}, R_{2}, S_{1}, S_{1}^{\prime}, S_{2}$, and $S_{2}^{\prime}$.

Apply the unitary transformation $T$ to the state in $\left(\mathrm{S}_{1}, \mathrm{~S}_{1}^{\prime}\right)$.

Prepare $|0\rangle$ in a single-qubit register $\mathrm{R}_{2}^{\prime}$.

2. Perform a phase-flip (i.e., multiply -1 in phase) if $\left(R_{1}, S_{1}\right)$ contains 11 .

3. Try to simulate Step 3 of the REFLECTION PROCEDURE by performing the following:

Apply $T^{\dagger}$ to the state in $\left(\mathrm{R}_{2}, \mathrm{R}_{2}^{\prime}\right)$. Measure the states in $\left(\mathrm{R}_{1}, \mathrm{R}_{2}\right)$ and $\left(\mathrm{S}_{1}, \mathrm{~S}_{2}\right)$ in the Bell basis. Continue if both of these two measurements result in $\left|\Phi^{+}\right\rangle$, and accept otherwise (accept with giving up due to failure of the simulation).

4. Reject if $\left(\mathrm{R}_{2}^{\prime}, \mathrm{S}_{2}^{\prime}\right)$ contains 00 , and accept otherwise.

Figure 5: The Reflection Simulation Test, which tries to simulate the Reflection Procedure using Choi-Jamiołkowski states.

Proposition 20. For any real number $q \in[0,1]$, the REFLECTION Simulation TeST results in rejection with probability $1 / 16$ if the state in the input register $\left(\mathrm{R}_{1}, \mathrm{R}_{2}, \mathrm{~S}_{1}, \mathrm{~S}_{1}^{\prime}, \mathrm{S}_{2}, \mathrm{~S}_{2}^{\prime}\right)$ is either $|0\rangle^{\otimes 2} \otimes\left|J\left(W_{q}^{+}\right)\right\rangle^{\otimes 2}$ or $|0\rangle^{\otimes 2} \otimes\left|J\left(W_{q}^{-}\right)\right\rangle^{\otimes 2}$, where $W_{q}^{+}=W_{q}$ and

$$
W_{q}^{-}=Z W_{q} Z=\left(\begin{array}{cc}
\sqrt{1-q} & -\sqrt{q} \\
-\sqrt{q} & -\sqrt{1-q}
\end{array}\right)=\sqrt{1-q} Z-\sqrt{q} X .
$$

Proof. We prove the case where the state in $\left(\mathrm{R}_{1}, \mathrm{R}_{2}, \mathrm{~S}_{1}, \mathrm{~S}_{1}^{\prime}, \mathrm{S}_{2}, \mathrm{~S}_{2}^{\prime}\right)$ is $|0\rangle^{\otimes 2} \otimes\left|J\left(W_{q}^{+}\right)\right\rangle^{\otimes 2}$. The other case is proved similarly, by noticing that $T\left|J\left(W_{q}^{-}\right)\right\rangle=\left(W_{q}^{-}|0\rangle\right) \otimes|0\rangle$ and $W_{q}^{-\dagger}=W_{q}^{-}$hold for any $q \in[0,1]$.

With $|0\rangle$ in $\mathrm{R}_{1}$ and $\left|J\left(W_{q}^{+}\right)\right\rangle=\left|J\left(W_{q}\right)\right\rangle$ in $\left(\mathrm{S}_{1}, \mathrm{~S}_{1}^{\prime}\right)$, Step 1 in the Reflection Simulation Test creates the state

$$
|0\rangle_{\mathrm{R}_{1}} \otimes\left|\chi_{q}\right\rangle \mathrm{S}_{1}
$$

in $\left(\mathrm{R}_{1}, \mathrm{~S}_{1}\right)$. For this state given, Step 2 in the Reflection Simulation Test does not change the state in $\left(\mathrm{R}_{1}, \mathrm{~S}_{1}\right)$ at all. As $|0\rangle=\left|\chi_{0}\right\rangle$, the application of $T^{\dagger}$ in Step 3 generates the Choi-Jamiołkowski state $\left|J\left(W_{0}\right)\right\rangle$ in $\left(\mathrm{R}_{2}, \mathrm{R}_{2}^{\prime}\right)$, and thus, one succeeds in Step 3 with probability $(1 / 4)^{2}=1 / 16$ in applying both of $W_{0}^{\dagger}=W_{0}$ and $W_{q}^{\dagger}=W_{q}$. If such an event occurs, the state in $\left(\mathrm{R}_{2}^{\prime}, \mathrm{S}_{2}^{\prime}\right)$ becomes $|0\rangle_{\mathrm{R}_{2}^{\prime}} \otimes|0\rangle_{\mathrm{S}_{2}^{\prime}}$, and thus, the test results in rejection with certainty.

Taking it into account that the test just stops and accepts with giving up when any of measurements in Step 3 fails in measuring $\left|\Phi^{+}\right\rangle$, the test results in rejection with probability $1 / 16$ in total.

\subsection{Proof of Theorem 2}

Now we are ready to prove Theorem 2 
Proof of Theorem 2 Let $A=\left(A_{\mathrm{yes}}, A_{\mathrm{no}}\right)$ be in QMA and let $V$ be the verifier of the corresponding QMA system. Without loss of generality, one can assume that both completeness and soundness errors are exponentially small in this QMA system.

For an input $x$, the quantum circuit $V_{x}$ of the verifier $V$ acts over a pair of two registers $\mathrm{A}$ of $v(|x|)$ qubits and $\mathrm{M}$ of $m(|x|)$ qubits, for some polynomially bounded functions $v, m: \mathbb{Z}^{+} \rightarrow \mathbb{N}$. This can be interpreted as $V_{x}$ expecting to receive a quantum witness $|w\rangle$ of $m(|x|)$ qubits in register $\mathrm{M}$, and using the $v(|x|)$ qubits in $\mathrm{A}$ as its work qubits. By Refs. [Shi02, Aha03], one can further assume that the quantum circuit $V_{x}$ for any input $x$ consists of only the Hadamard, Toffoli, and NOT gates. As pointed out by Marriott and Watrous [MW05], the maximum acceptance probability $p_{x}$ of $V$ with input $x$ corresponds to the maximum eigenvalue of the Hermitian operator

$$
M_{x}=\Pi_{\text {init }} V_{x}^{\dagger} \Pi_{\text {acc }} V_{x} \Pi_{\text {init }},
$$

where $\Pi_{\text {init }}$ is the projection onto the subspace spanned by states in which all the qubits in $A$ are in state $|0\rangle$, and $\Pi_{\text {acc }}$ is the projection onto the space spanned by the accepting states of $V$. From this verifier $V$, we shall construct a protocol for the verifier $W$ of another QMA system in which $W$ shares $N$ EPR pairs a priori with a prover communicating with, where $N$ is a constant that is a power of two.

Our basic strategy is to try to perform the REFLECTION Simulation Test using $V_{x}$. Fix an input $x$, and let $p=\frac{p_{x}^{2}}{2 p_{x}^{2}-2 p_{x}+1}$. Let $\mathrm{S}_{1}, \ldots, \mathrm{S}_{N}$ be single-qubit registers which store the particles of the shared EPR pairs. In addition to $\mathrm{M}, W$ receives $N$ single-qubit registers $\mathrm{S}_{1}^{\prime}, \ldots, \mathrm{S}_{N}^{\prime}$. $W$ expects to receive in $\mathrm{M}$ the state $\left|w_{x}\right\rangle$ that is the eigenstate (i.e., eigenvector) of $M_{x}$ corresponding to the eigenvalue $p_{x}$, and to receive states in $\mathrm{S}_{1}^{\prime}, \ldots, \mathrm{S}_{N}^{\prime}$ such that the state in $\left(\mathrm{S}_{j}, \mathrm{~S}_{j}^{\prime}\right)$ forms $\left|J\left(W_{q}\right)\right\rangle$ for each $j \in\{1, \ldots, N\}$, for $q$ satisfying $p q=\frac{p_{x}^{2}}{2 p_{x}^{2}-2 p_{x}+1} q=1 / 2$. In addition to $A, W$ prepares three single-qubit registers $B, R_{1}$, and $R_{2}$. All the qubits in $A, B, R_{1}$, and $R_{2}$ are initialized to the $|0\rangle$ state.

First, $W$ performs the Distillation Procedure twice in sequence, first with $\left(\mathrm{R}_{1}, \mathrm{~A}, \mathrm{M}\right)$ as input, and second with $\left(\mathrm{R}_{2}, \mathrm{~A}, \mathrm{M}\right)$ as input. If any of these two runs of the Distillation Procedure outputs a symbol $\perp$, the simulation fails, and thus accept with giving up. If not failed, then $W$ chooses two indices $r_{1}$ and $r_{2}$ from the set $\{1, \ldots, N\}$ uniformly at random. If $r_{2}=1, W$ accepts with giving up. Otherwise $W$ swaps the registers $\left(\mathrm{S}_{1}, \mathrm{~S}_{1}^{\prime}\right)$ and $\left(\mathrm{S}_{r_{1}}, \mathrm{~S}_{r_{1}}^{\prime}\right)$ if $r_{1} \geq 2$, and further swaps $\left(\mathrm{S}_{2}, \mathrm{~S}_{2}^{\prime}\right)$ and $\left(\mathrm{S}_{r_{2}}, \mathrm{~S}_{r_{2}}^{\prime}\right)$ if $r_{2} \geq 3$. Afterwards, $W$ never touches the registers $\left(\mathrm{S}_{j}, \mathrm{~S}_{j}^{\prime}\right)$ for $j \geq 3$, and thus this process essentially has the same effect as performing a random permutation over the registers $\left(\mathrm{S}_{1}, \mathrm{~S}_{1}^{\prime}\right), \ldots,\left(\mathrm{S}_{N}, \mathrm{~S}_{N}^{\prime}\right)$. $W$ then performs the SPACE Restriction Test by checking if the state in $\left(S_{j}, S_{j}^{\prime}\right)$ is in the space spanned by $\left\{\left|\Phi^{-}\right\rangle,\left|\Psi^{+}\right\rangle\right\}$, for each $j \in\{1,2\}$, and further performs the SWAP TEST between $\left(\mathrm{S}_{1}, \mathrm{~S}_{1}^{\prime}\right)$ and $\left(\mathrm{S}_{2}, \mathrm{~S}_{2}^{\prime}\right)$ (using the register $\mathrm{B}$ as the control). Finally, $W$ performs the Reflection Simulation Test with $\left(R_{1}, R_{2}, S_{1}, S_{1}^{\prime}, S_{2}, S_{2}^{\prime}\right)$ as input. The protocol is summarized in Figure 6 Notice that this protocol is exactly implementable when the Hadamard and any classical reversible transformations can be performed exactly.

For the completeness, suppose that $x$ is in $A_{\text {yes }}$. Let $p=\frac{p_{x}^{2}}{2 p_{x}^{2}-2 p_{x}+1}$. The honest Merlin sets his shares of the $N$ EPR pairs in single-qubit registers $\mathrm{S}_{1}^{\prime}, \ldots, \mathrm{S}_{N}^{\prime}$, and applies $W_{q}$ to each qubit in $\left(\mathrm{S}_{1}^{\prime}, \ldots, \mathrm{S}_{N}^{\prime}\right)$ to create the state $\left|J\left(W_{q}\right)\right\rangle$ in $\left(\mathrm{S}_{j}, \mathrm{~S}_{j}^{\prime}\right)$, for $j \in\{1, \ldots, N\}$, where $q$ satisfies $p q=1 / 2$ (such a $q$ always exists when $p_{x} \geq 1 / 2$, which is ensured by the completeness condition of the original QMA system). He also prepares $\left|w_{x}\right\rangle$ in $\mathbf{M}$, and sends the $(m(|x|)+N)$-qubit state in $\left(\mathrm{M}, \mathrm{S}_{1}^{\prime}, \ldots, \mathrm{S}_{N}^{\prime}\right)$ as a witness. Then, conditioned on the first application of the Distillation Procedure not outputting $\perp$, the state $\left|\chi_{p}\right\rangle=W_{p}|0\rangle$ is generated in $\mathrm{R}_{1}$, and $|0\rangle^{\otimes v(|x|)} \otimes\left|w_{x}\right\rangle$ is left in $(A, M)$, and thus, the state $\left|\chi_{p}\right\rangle$ is generated also in $R_{2}$ when the second application of the DistiLlation Procedure does not output $\perp$. Conditioned on the chosen $r_{2}$ not being 1 in Step 3, the protocol continues and the state remains the same after this step. When continued, the SPACE RESTRICTION TEST in Step 4 clearly never rejects and does not change the state at all, as the state in $\left(\mathrm{S}_{j}, \mathrm{~S}_{j}^{\prime}\right)$ is $\left|J\left(W_{q}\right)\right\rangle=\sqrt{1-q}\left|\Phi^{-}\right\rangle+\sqrt{q}\left|\Psi^{+}\right\rangle$ for each $j \in\{1,2\}$. Furthermore, the SWAP TEST never fails in Step 5 and it does not change the state at all (and thus, the protocol never results in rejection in this step). Therefore, the state in $\left(R_{1}, R_{2}, S_{1}, S_{1}^{\prime}, S_{2}, S_{2}^{\prime}\right)$ is $\left|\chi_{p}\right\rangle^{\otimes 2} \otimes\left|J\left(W_{q}\right)\right\rangle^{\otimes 2}$, when entering Step 6. Hence, from Proposition 19, the Reflection Simulation 


\section{Verifier's QMA Protocol for Achieving Perfect Completeness with $N$ Prior-Shared EPR Pairs}

1. Store the particles of the shared $N$ EPR pairs in $\left(\mathrm{S}_{1}, \ldots, \mathrm{S}_{N}\right)$. Receive an $(m(|x|)+N)$-qubit quantum witness in $\left(\mathrm{M}, \mathrm{S}_{1}^{\prime}, \ldots, \mathrm{S}_{N}^{\prime}\right)$, where the first $m(|x|)$ qubits of the witness are in $\mathbf{M}$, and the $(m(|x|)+j)$-th qubit of the witness is in $\mathrm{S}_{j}$, for $j \in\{1, \ldots, N\}$.

Prepare $|0\rangle$ in each of the three single-qubit registers $\mathrm{B}, \mathrm{R}_{1}$ and $\mathrm{R}_{2}$, and $|0\rangle{ }^{\otimes v(|x|)}$ in a $v(|x|)$-qubit register $\mathrm{A}$, which corresponds to the private space of the original verifier.

2. Execute the Distillation Procedure with $\left(R_{1}, A, M\right)$ as input. Accept if this outputs $\perp$, and continue otherwise. Execute the Distillation Procedure again, this time using $\left(R_{2}, A, M\right)$ as input. Accept if this outputs $\perp$, and continue otherwise.

3. Choose two integers $r_{1}$ and $r_{2}$ from $\{1, \ldots, N\}$ uniformly at random. Accept if $r_{2}=1$ (accept with giving up due to failure of simulation), and continue otherwise. Swap the registers $\left(\mathrm{S}_{1}, \mathrm{~S}_{1}^{\prime}\right)$ and $\left(\mathrm{S}_{r_{1}}, \mathrm{~S}_{r_{1}}^{\prime}\right)$ if $r_{1} \geq 2$, and further swap the registers $\left(\mathrm{S}_{2}, \mathrm{~S}_{2}^{\prime}\right)$ and $\left(\mathrm{S}_{r_{2}}, \mathrm{~S}_{r_{2}}^{\prime}\right)$ if $r_{2} \geq 3$.

4. Perform the Space Restriction Test to check if the state in $\left(S_{j}, S_{j}^{\prime}\right)$ is in the space spanned by $\left\{\left|\Phi^{-}\right\rangle,\left|\Psi^{+}\right\rangle\right\}$, for each $j \in\{1,2\}$. Reject if not so, and continue otherwise.

That is, perform the following for each $j \in\{1,2\}$ : Apply the unitary transformation $T$ defined by

$$
T:\left|\Phi^{-}\right\rangle \mapsto|00\rangle,\left|\Psi^{-}\right\rangle \mapsto|01\rangle,\left|\Psi^{+}\right\rangle \mapsto|10\rangle,\left|\Phi^{+}\right\rangle \mapsto|11\rangle
$$

to the state in $\left(\mathrm{S}_{j}, \mathrm{~S}_{j}^{\prime}\right)$. Reject if $\mathrm{S}_{j}^{\prime}$ contains 1 , and apply $T^{\dagger}$ to the state in $\left(\mathrm{S}_{j}, \mathrm{~S}_{j}^{\prime}\right)$ to continue otherwise.

5. Perform the SwAP TEST between $\left(\mathrm{S}_{1}, \mathrm{~S}_{1}^{\prime}\right)$ and $\left(\mathrm{S}_{2}, \mathrm{~S}_{2}^{\prime}\right)$. Reject if it fails, and continue otherwise.

That is, apply $H$ to $\mathrm{B}$, swap $\left(\mathrm{S}_{1}, \mathrm{~S}_{1}^{\prime}\right)$ and $\left(\mathrm{S}_{2}, \mathrm{~S}_{2}^{\prime}\right)$ if $\mathrm{B}$ contains 1 , apply $H$ to $\mathrm{B}$ again, and reject if $\mathrm{B}$ contains 1 , and continue otherwise.

6. Perform the Reflection Simulation Test with $\left(R_{1}, R_{2}, S_{1}, S_{1}^{\prime}, S_{2}, S_{2}^{\prime}\right)$ as input. Accept if this returns "accept", and reject otherwise.

Figure 6: Verifier's QMA protocol for achieving perfect completeness with $N$ pre-shared EPR pairs.

TEST results in acceptance with certainty, when the protocol reaches Step 6. As rejections can happen only in Steps 4,5 , and 6 , this proves the perfect completeness.

Now for the soundness, suppose that $x$ is in $A_{\text {no. Let }} \mathcal{R}_{j}, \mathcal{S}_{j}$, and $\mathcal{S}_{j}^{\prime}$ denote the Hilbert spaces associated with the quantum registers $\mathrm{R}_{j}, \mathrm{~S}_{j}$, and $\mathrm{S}_{j}^{\prime}$, for each $j$, respectively.

As the soundness error of the original QMA system is exponentially small, whatever state the register M contains, the probability that the first application of the DISTILLATION PROCEDURE outputs $\perp$ is exponentially small. Moreover, conditioned on this not outputting $\perp$, the state generated in $R_{1}$ is exponentially close to $|0\rangle$ (in trace distance). Similarly, whatever state left in $M$ after the first application of the DisTILlation ProceduRE, the probability that the second application of the DisTILLATION PROCEDURE outputs $\perp$ is exponentially small, and the state generated in $\mathrm{R}_{2}$ is exponentially close to $|0\rangle$. Hence, the state in $\left(\mathrm{R}_{1}, \mathrm{R}_{2}, \mathrm{~S}_{1}, \mathrm{~S}_{1}^{\prime}, \ldots, \mathrm{S}_{N}, \mathrm{~S}_{N}^{\prime}\right)$ when entering Step 2 must be exponentially close to $(|0\rangle\langle 0|)^{\otimes 2} \otimes \rho$ for some $2 N$-qubit state $\rho$ such that the reduced state

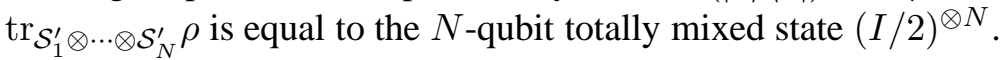

As Step 3 essentially has the same effect as performing a random permutation over the registers $\left(\mathrm{S}_{1}, \mathrm{~S}_{1}^{\prime}\right), \ldots,\left(\mathrm{S}_{N}, \mathrm{~S}_{N}^{\prime}\right)$ for the purpose of computing the reduced state in $\left(\mathrm{S}_{1}, \mathrm{~S}_{1}^{\prime}, \mathrm{S}_{2}, \mathrm{~S}_{2}^{\prime}\right)$, from the finite quantum de Finetti theorem (Theorem 8 ), the state in $\left(R_{1}, R_{2}, S_{1}, S_{1}^{\prime}, S_{2}, S_{2}^{\prime}\right)$ after Step 3 should have trace distance at 
most $\frac{2^{6}}{N}$ to the state

$$
\sigma=(|0\rangle\langle 0|)^{\otimes 2} \otimes\left(\sum_{j} \mu_{j} \xi_{j}^{\otimes 2}\right)
$$

for some two-qubit states $\xi_{j}$, where $\sum_{j} \mu_{j}=1$, if the state in $\left(\mathrm{R}_{1}, \mathrm{R}_{2}, \mathrm{~S}_{1}, \mathrm{~S}_{1}^{\prime}, \ldots, \mathrm{S}_{N}, \mathrm{~S}_{N}^{\prime}\right)$ were $(|0\rangle\langle 0|)^{\otimes 2} \otimes \rho$ when entering Step 3 and if $r_{2} \neq 1$ (here we are taking the randomness over the choices of $r_{1}$ and $r_{2}$ into account). By letting $\tau=\sum_{j} \mu_{j} \xi_{j}^{\otimes 2}$, this in particular implies that for the reduced state $\operatorname{tr}_{\mathcal{S}_{1}^{\prime} \otimes \mathcal{S}_{2}^{\prime}} \tau$ and the two-qubit totally mixed state $(I / 2)^{\otimes 2}$,

$$
D\left(\operatorname{tr}_{\mathcal{S}_{1}^{\prime} \otimes \mathcal{S}_{2}^{\prime}} \tau,\left(\frac{I}{2}\right)^{\otimes 2}\right) \leq \frac{2^{6}}{N}
$$

holds, since $\operatorname{tr}_{\mathcal{S}_{1}^{\prime} \otimes \cdots \otimes \mathcal{S}_{N}^{\prime}} \rho=(I / 2)^{\otimes N}$. Taking it into account that the protocol enters Step 3 with probability exponentially close to 1 with the state in $\left(\mathrm{R}_{1}, \mathrm{R}_{2}, \mathrm{~S}_{1}, \mathrm{~S}_{1}^{\prime}, \ldots, \mathrm{S}_{N}, \mathrm{~S}_{N}^{\prime}\right)$ being exponentially close to $(|0\rangle\langle 0|)^{\otimes 2} \otimes \rho$ in trace distance, we conclude that the protocol enters Step 4 with probability exponentially close to $1-\frac{1}{N}$ with the state in $\left(\mathrm{R}_{1}, \mathrm{R}_{2}, \mathrm{~S}_{1}, \mathrm{~S}_{1}^{\prime}, \mathrm{S}_{2}, \mathrm{~S}_{2}^{\prime}\right)$ having trace distance at most $\frac{2^{6}}{N}+\varepsilon$ to $\sigma$ for some exponentially small $\varepsilon$.

Now from Proposition 21 which will be found below and proved in the end of this section, the protocol should result in rejection with probability at least $\min \left\{\frac{2^{7}}{N}, \frac{1}{16}-15\left(\frac{2^{6}}{N}\right)^{\frac{1}{8}}\right\}$ if the state in $\left(\mathrm{R}_{1}, \mathrm{R}_{2}, \mathrm{~S}_{1}, \mathrm{~S}_{1}^{\prime}, \mathrm{S}_{2}, \mathrm{~S}_{2}^{\prime}\right)$ were $\sigma$ when entering Step 4. Hence, using Lemma 6 the protocol results in rejection with probability at least $\min \left\{\frac{2^{6}}{N}-\varepsilon, \frac{1}{16}-\frac{2^{6}}{N}-\varepsilon-15\left(\frac{2^{6}}{N}\right)^{\frac{1}{8}}\right\}$, when entering Step 4. As the protocol enters Step 4 with probability exponentially close to $1-\frac{1}{N}$, by taking $N=2^{70}$, the protocol results in rejection with probability at least

$$
\left(1-\frac{1}{2^{69}}\right) \cdot \min \left\{\frac{1}{2^{65}}, \frac{1}{16}-\frac{1}{2^{63}}-\frac{15}{2^{8}}\right\} \geq \frac{1}{2^{66}} .
$$

This proves the inclusion

$$
\mathrm{QMA} \subseteq \mathrm{QMA}^{2^{70}-\mathrm{EPR}}\left(1,1-\frac{1}{2^{66}}\right)
$$

Now for any constant $s \in(0,1)$, one can achieve soundness $s$ simply by repeating this proof system $t$ times in parallel for some appropriate constant $t$, as the system is a special case of two-message quantum interactive proof systems, for which parallel repetition works perfectly [KW00]. This completes the proof.

Finally, we prove the following proposition.

Proposition 21. When entering Step 4 of the protocol described in Figure 6 suppose that the state in $\left(\mathrm{R}_{1}, \mathrm{R}_{2}, \mathrm{~S}_{1}, \mathrm{~S}_{1}^{\prime}, \mathrm{S}_{2}, \mathrm{~S}_{2}^{\prime}\right)$ were of the form $(|0\rangle\langle 0|)^{\otimes 2} \otimes \tau$ where $\tau=\sum_{j} \mu_{j} \xi_{j}^{\otimes 2}$ for some two-qubit states $\xi_{j}$ and real numbers $\mu_{j} \in[0,1]$ satisfying $\sum_{j} \mu_{j}=1$, such that the reduced state of $\tau$ in $\left(\mathrm{S}_{1}, \mathrm{~S}_{2}\right)$ has trace distance at most $\delta$ to the two-qubit totally mixed state $(I / 2)^{\otimes 2}$ for some positive $\delta$ satisfying $\frac{1}{16}-15 \delta^{\frac{1}{8}}>0$. Then the protocol should result in rejection with probability at least $\min \left\{2 \delta, \frac{1}{16}-15 \delta^{\frac{1}{8}}\right\}$.

To prove Proposition 21, we first show two propositions that are special cases of Proposition 21 .

Proposition 22. Let $\mathcal{W}$ be the two-dimensional space spanned by $\left|\Phi^{-}\right\rangle$and $\left|\Psi^{+}\right\rangle$. When entering Step 4 of the protocol described in Figure 6 suppose that the state in $\left(\mathrm{R}_{1}, \mathrm{R}_{2}, \mathrm{~S}_{1}, \mathrm{~S}_{1}^{\prime}, \mathrm{S}_{2}, \mathrm{~S}_{2}^{\prime}\right)$ were of the form $(|0\rangle\langle 0|)^{\otimes 2} \otimes \tau$ where $\tau=\sum_{j} \mu_{j}\left(\left|\psi_{j}\right\rangle\left\langle\psi_{j}\right|\right)^{\otimes 2}$ for some two-qubit states $\left|\psi_{j}\right\rangle \in \mathcal{W}$ and real numbers $\mu_{j} \in[0,1]$ satisfying $\sum_{j} \mu_{j}=1$, such that the reduced state of $\tau$ in $\left(\mathrm{S}_{1}, \mathrm{~S}_{2}\right)$ has trace distance at most $\delta$ to the two-qubit totally mixed state $(I / 2)^{\otimes 2}$ for some positive $\delta$ satisfying $\frac{1}{16}-\frac{\pi}{2} \delta^{\frac{1}{2}}>0$. Then the protocol should result in rejection with probability at least $\frac{1}{16}-\frac{\pi}{2} \delta^{\frac{1}{2}}$.

The following lemma is essential for the proof of Proposition 22. 
Lemma 23. For each $j \in\{1,2\}$, let $\mathcal{S}_{j}$ and $\mathcal{S}_{j}^{\prime}$ be two-dimensional complex Hilbert spaces $\mathbb{C}(\Sigma)$, and let $\mathcal{W}_{j}$ be the two-dimensional subspace of $\mathcal{S}_{j} \otimes \mathcal{S}_{j}^{\prime}$ spanned by $\left|\Phi^{-}\right\rangle$and $\left|\Psi^{+}\right\rangle$. Let $\rho$ be any four-qubit state in $\mathbf{D}\left(\mathcal{W}_{1} \otimes \mathcal{W}_{2}\right) \subseteq \mathbf{D}\left(\mathcal{S}_{1} \otimes \mathcal{S}_{1}^{\prime} \otimes \mathcal{S}_{2} \otimes \mathcal{S}_{2}^{\prime}\right)$ that is a mixture of two-fold product pure states $\left|\zeta_{j}\right\rangle^{\otimes 2}$ in $\mathcal{W}_{1} \otimes \mathcal{W}_{2}$ and such that $D\left(\operatorname{tr}_{\mathcal{S}_{1}^{\prime} \otimes \mathcal{S}_{2}^{\prime}} \rho,(I / 2)^{\otimes 2}\right) \leq \delta$. Then there exists a four-qubit state $\sigma$ that is a mixture of two-fold products $\left|J\left(W_{a_{j}}^{ \pm}\right)\right\rangle^{\otimes 2}$ of a Choi-Jamiotkowski state, for real numbers $a_{j} \in[0,1]$, such that $D(\rho, \sigma) \leq \frac{\pi}{2} \delta^{\frac{1}{2}}$, where each $W_{a_{j}}^{ \pm}$is equal to either $W_{a_{j}}^{+}=W_{a_{j}}$ or $W_{a_{j}}^{-}=Z W_{a_{j}} Z$.

Proof. As $\rho$ is a mixture of two-fold product pure states in $\mathcal{W}_{1} \otimes \mathcal{W}_{2}$, it must be written as

$$
\rho=\sum_{j} \mu_{j}\left(\left|\zeta_{j}\right\rangle\left\langle\zeta_{j}\right|\right)^{\otimes 2}
$$

where $\left|\zeta_{j}\right\rangle^{\otimes 2} \in \mathcal{W}_{1} \otimes \mathcal{W}_{2}, \mu_{j} \in[0,1]$ for each $j$, and $\sum_{j} \mu_{j}=1$. Without loss of generality, one may assume that

$$
\left|\zeta_{j}\right\rangle=\alpha_{j}\left|\Phi^{-}\right\rangle+\beta_{j} e^{i \theta_{j}}\left|\Psi^{+}\right\rangle
$$

for each $j$, where $\alpha_{j}$ and $\beta_{j}$ are real numbers in $[0,1]$ satisfying $\alpha_{j}^{2}+\beta_{j}^{2}=1$, and $\theta_{j}$ is a real number in $[0,2 \pi)$. For each $j$, let $a_{j}=\beta_{j}^{2}$, and define the two-qubit pure state $\left|\eta_{j}\right\rangle$ as

$$
\left|\eta_{j}\right\rangle=\alpha_{j}\left|\Phi^{-}\right\rangle+\beta_{j}\left|\Psi^{+}\right\rangle=\sqrt{1-a_{j}}\left|\Phi^{-}\right\rangle+\sqrt{a_{j}}\left|\Psi^{+}\right\rangle=\left|J\left(W_{a_{j}}^{+}\right)\right\rangle
$$

if $j \in J_{+}$, and

$$
\left|\eta_{j}\right\rangle=\alpha_{j}\left|\Phi^{-}\right\rangle-\beta_{j}\left|\Psi^{+}\right\rangle=\sqrt{1-a_{j}}\left|\Phi^{-}\right\rangle-\sqrt{a_{j}}\left|\Psi^{+}\right\rangle=\left|J\left(W_{a_{j}}^{-}\right)\right\rangle
$$

if $j \in J_{-}$, where $J_{+}=\left\{j: \theta_{j} \in[0, \pi / 2] \cup[3 \pi / 2,2 \pi)\right\}$ and $J_{-}=\left\{j: \theta_{j} \in(\pi / 2,3 \pi / 2)\right\}$.

Now take the four-qubit state $\sigma$ as

$$
\sigma=\sum_{j} \mu_{j}\left(\left|\eta_{j}\right\rangle\left\langle\eta_{j}\right|\right)^{\otimes 2}
$$

We shall show that this $\sigma$ has the desired property. For this purpose, we prove two claims.

Claim 1. $D\left(\operatorname{tr}_{\mathcal{S}_{1}^{\prime} \otimes \mathcal{S}_{2}^{\prime}} \rho,(I / 2)^{\otimes 2}\right) \geq 2 \sum_{j} \mu_{j} \alpha_{j}^{2} \beta_{j}^{2} \sin ^{2} \theta_{j}$.

Proof. Noticing that

$$
\begin{aligned}
\left|\zeta_{j}\right\rangle & =\frac{1}{\sqrt{2}}\left[\alpha_{j}(|00\rangle-|11\rangle)+\beta_{j} e^{i \theta_{j}}(|01\rangle+|10\rangle)\right] \\
& =\frac{1}{\sqrt{2}}\left[\left(\alpha_{j}|0\rangle+\beta_{j} e^{i \theta_{j}}|1\rangle\right) \otimes|0\rangle+e^{i \theta_{j}}\left(\beta_{j}|0\rangle-\alpha_{j} e^{-i \theta_{j}}|1\rangle\right) \otimes|1\rangle\right],
\end{aligned}
$$

the reduced state $\operatorname{tr}_{\mathcal{S}_{1}^{\prime} \otimes \mathcal{S}_{2}^{\prime}} \rho$ is the mixture of the following four states

$$
\begin{aligned}
& \left(\alpha_{j}|0\rangle+\beta_{j} e^{i \theta_{j}}|1\rangle\right) \otimes\left(\alpha_{j}|0\rangle+\beta_{j} e^{i \theta_{j}}|1\rangle\right), \\
& \left(\alpha_{j}|0\rangle+\beta_{j} e^{i \theta_{j}}|1\rangle\right) \otimes\left(\beta_{j}|0\rangle-\alpha_{j} e^{-i \theta_{j}}|1\rangle\right), \\
& \left(\beta_{j}|0\rangle-\alpha_{j} e^{-i \theta_{j}}|1\rangle\right) \otimes\left(\alpha_{j}|0\rangle+\beta_{j} e^{i \theta_{j}}|1\rangle\right), \\
& \left(\beta_{j}|0\rangle-\alpha_{j} e^{-i \theta_{j}}|1\rangle\right) \otimes\left(\beta_{j}|0\rangle-\alpha_{j} e^{-i \theta_{j}}|1\rangle\right)
\end{aligned}
$$

with equal probability $1 / 4$ for each, which can be expressed as a density matrix by

$$
\frac{1}{4}\left(\begin{array}{cccc}
1 & -2 i \alpha_{j} \beta_{j} s_{j} & -2 i \alpha_{j} \beta_{j} s_{j} & -4 \alpha_{j}^{2} \beta_{j}^{2} s_{j}^{2} \\
2 i \alpha_{j} \beta_{j} s_{j} & 1 & 4 \alpha_{j}^{2} \beta_{j}^{2} s_{j}^{2} & -2 i \alpha_{j} \beta_{j} s_{j} \\
2 i \alpha_{j} \beta_{j} s_{j} & 4 \alpha_{j}^{2} \beta_{j}^{2} s_{j}^{2} & 1 & -2 i \alpha_{j} \beta_{j} s_{j} \\
-4 \alpha_{j}^{2} \beta_{j}^{2} s_{j}^{2} & 2 i \alpha_{j} \beta_{j} s_{j} & 2 i \alpha_{j} \beta_{j} s_{j} & 1
\end{array}\right),
$$




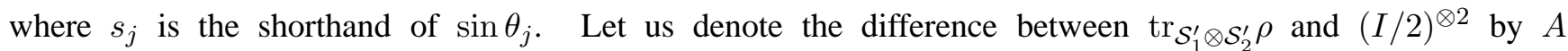
(i.e., $A=\operatorname{tr}_{\mathcal{S}_{1}^{\prime} \otimes \mathcal{S}_{2}^{\prime}} \rho-(I / 2)^{\otimes 2}$ ). In order to find the eigenvalues of $2 A$, we solve the characteristic equation $|2 A-\lambda I|=0$. Straightforward calculations show that the four solutions of the equation $|2 A-\lambda I|=0$ are given by $-2 \sum_{j} \mu_{j} \alpha_{j}^{2} \beta_{j}^{2} s_{j}^{2}$ (two-fold) and $2 \sum_{j} \mu_{j} \alpha_{j}^{2} \beta_{j}^{2} s_{j}^{2} \pm 2\left|\sum_{j} \mu_{j} \alpha_{j} \beta_{j} s_{j}\right|$. This implies that

$$
\begin{aligned}
& D\left(\operatorname{tr}_{\mathcal{S}_{1}^{\prime} \otimes \mathcal{S}_{2}^{\prime}} \rho,\left(\frac{I}{2}\right)^{\otimes 2}\right)=\frac{1}{2} \operatorname{tr} \sqrt{A^{\dagger} A} \\
& =\frac{1}{2}\left[2 \sum_{j} \mu_{j} \alpha_{j}^{2} \beta_{j}^{2} s_{j}^{2}+\left(\sum_{j} \mu_{j} \alpha_{j}^{2} \beta_{j}^{2} s_{j}^{2}+\left|\sum_{j} \mu_{j} \alpha_{j} \beta_{j} s_{j}\right|\right)+\left|\sum_{j} \mu_{j} \alpha_{j}^{2} \beta_{j}^{2} s_{j}^{2}-\right| \sum_{j} \mu_{j} \alpha_{j} \beta_{j} s_{j}||\right] \\
& =\sum_{j} \mu_{j} \alpha_{j}^{2} \beta_{j}^{2} s_{j}^{2}+\max \left\{\sum_{j} \mu_{j} \alpha_{j}^{2} \beta_{j}^{2} s_{j}^{2},\left|\sum_{j} \mu_{j} \alpha_{j} \beta_{j} s_{j}\right|\right\} \text {, }
\end{aligned}
$$

which is at least $2 \sum_{j} \mu_{j} \alpha_{j}^{2} \beta_{j}^{2} s_{j}^{2}$. This completes the proof of the claim.

Claim 2. Let $\left\{\mu_{j}\right\}$ be a probability distribution, and $\left\{c_{j}\right\}$ be a set of real numbers. If $\sum_{j} \mu_{j} c_{j}^{2} \leq \varepsilon$, it holds that $\sum_{j} \mu_{j}\left|c_{j}\right| \leq \varepsilon^{\frac{1}{2}}$.

Proof. By the Cauchy-Schwarz inequality, we have

$$
\sum_{j} \mu_{j}\left|c_{j}\right|=\sum_{j} \sqrt{\mu_{j}} \cdot \sqrt{\mu_{j}}\left|c_{j}\right| \leq\left(\sum_{j} \mu_{j}\right)^{\frac{1}{2}}\left(\sum_{j} \mu_{j}\left|c_{j}\right|^{2}\right)^{\frac{1}{2}} \leq \varepsilon^{\frac{1}{2}}
$$

as claimed.

Now we bound $D(\rho, \sigma)$. Notice that

$$
D(\rho, \sigma) \leq \sum_{j} \mu_{j} D\left(\left(\left|\zeta_{j}\right\rangle\left\langle\zeta_{j}\right|\right)^{\otimes 2},\left(\left|\eta_{j}\right\rangle\left\langle\eta_{j}\right|\right)^{\otimes 2}\right)=\sum_{j \in J_{+}} \mu_{j} \sqrt{1-\left|\left\langle\zeta_{j} \mid \eta_{j}\right\rangle\right|^{4}}+\sum_{j \in J_{-}} \mu_{j} \sqrt{1-\left|\left\langle\zeta_{j} \mid \eta_{j}\right\rangle\right|^{4}} .
$$

If $j \in J_{+}$, it holds that

$$
\left|\left\langle\zeta_{j} \mid \eta_{j}\right\rangle\right|^{4}=\left|\alpha_{j}^{2}+\beta_{j}^{2} e^{-i \theta_{j}}\right|^{4}=\left[\left(\alpha_{j}^{2}+\beta_{j}^{2} \cos \theta_{j}\right)^{2}+\left(\beta_{j}^{2} \sin \theta_{j}\right)^{2}\right]^{2}=\left(1-4 \alpha_{j}^{2} \beta_{j}^{2} \sin ^{2} \frac{\theta_{j}}{2}\right)^{2},
$$

and thus,

$$
\sqrt{1-\left|\left\langle\zeta_{j} \mid \eta_{j}\right\rangle\right|^{4}}=2 \sqrt{2}\left|\alpha_{j} \beta_{j} \sin \frac{\theta_{j}}{2}\right| \sqrt{1-2 \alpha_{j}^{2} \beta_{j}^{2} \sin ^{2} \frac{\theta_{j}}{2}} \leq 2 \sqrt{2}\left|\alpha_{j} \beta_{j} \sin \frac{\theta_{j}}{2}\right| \leq \frac{\pi}{\sqrt{2}}\left|\alpha_{j} \beta_{j} \sin \theta_{j}\right|,
$$

where the last inequality comes from the fact that for any $\theta \in[0, \pi / 2] \cup[3 \pi / 2,2 \pi),\left|\sin \frac{\theta}{2}\right| \leq\left|\frac{\theta}{2}\right| \leq \frac{\pi}{4}|\sin \theta|$.

On the other hand, if $j \in J_{-}$, we have

$$
\left|\left\langle\zeta_{j} \mid \eta_{j}\right\rangle\right|^{4}=\left|\alpha_{j}^{2}-\beta_{j}^{2} e^{-i \theta_{j}}\right|^{4}=\left|\alpha_{j}^{2}+\beta_{j}^{2} e^{-i \theta_{j}^{\prime}}\right|^{4}
$$

where $\theta_{j}^{\prime}=\theta_{j}+\pi(\bmod 2 \pi)$. Noticing that $\theta_{j}^{\prime} \in[0, \pi / 2] \cup[3 \pi / 2,2 \pi)$, it holds that

$$
\sqrt{1-\left|\left\langle\zeta_{j} \mid \eta_{j}\right\rangle\right|^{4}} \leq \frac{\pi}{\sqrt{2}}\left|\alpha_{j} \beta_{j} \sin \theta_{j}^{\prime}\right| \text {. }
$$


Therefore,

$$
D(\rho, \sigma) \leq \frac{\pi}{\sqrt{2}} \sum_{j} \mu_{j}\left|c_{j}\right|
$$

where

$$
c_{j}= \begin{cases}\alpha_{j} \beta_{j} \sin \theta_{j} & \text { if } j \in J_{+}, \\ \alpha_{j} \beta_{j} \sin \theta_{j}^{\prime} & \text { if } j \in J_{-} .\end{cases}
$$

By Claim 1 and the fact that $\sin ^{2} \theta_{j}^{\prime}=\sin ^{2} \theta_{j}$ for each $j \in J_{-}$, the assumption $D\left(\operatorname{tr}_{\mathcal{S}_{1}^{\prime} \otimes \mathcal{S}_{2}^{\prime}} \rho,(I / 2)^{\otimes 2}\right) \leq \delta$ implies that

$$
\sum_{j} \mu_{j} c_{j}^{2}=\sum_{j} \mu_{j} \alpha_{j}^{2} \beta_{j}^{2} \sin ^{2} \theta_{j} \leq \frac{\delta}{2} .
$$

By Claim 2, this implies that $D(\rho, \sigma) \leq \frac{\pi}{\sqrt{2}}\left(\frac{\delta}{2}\right)^{\frac{1}{2}}=\frac{\pi}{2} \delta^{\frac{1}{2}}$, which completes the proof of Lemma 23.

Proof of Proposition 22 Let $\sigma=(|0\rangle\langle 0|)^{\otimes 2} \otimes \tau$. From Lemma 23, there exists a quantum state $\tau^{\prime}$ that is a mixture of two-fold products $\left|J\left(W_{a_{j}}^{ \pm}\right)\right\rangle^{\otimes 2}$ of a Choi-Jamiołkowski state, for real numbers $a_{j} \in[0,1]$, such that, for $\sigma^{\prime}=(|0\rangle\langle 0|)^{\otimes 2} \otimes \tau^{\prime}, D\left(\sigma, \sigma^{\prime}\right) \leq \frac{\pi}{2} \delta^{\frac{1}{2}}$. Here, as in Lemma 23, each $W_{a_{j}}^{ \pm}$is equal to either $W_{a_{j}}^{+}=W_{a_{j}}$ or $W_{a_{j}}^{-}=Z W_{a_{j}} Z$. From Proposition 20, the REFLECTION Simulation Test should result in rejection with probability $\frac{1}{16}$ if the quantum state in $\left(\mathrm{R}_{1}, \mathrm{R}_{2}, \mathrm{~S}_{1}, \mathrm{~S}_{1}^{\prime}, \mathrm{S}_{2}, \mathrm{~S}_{2}^{\prime}\right)$ were $\sigma^{\prime}$. By Lemma 6 this implies that the REFLECTION SIMULATION TEST should result in rejection with probability at least $\frac{1}{16}-\frac{\pi}{2} \delta^{\frac{1}{2}}$ if the state in $\left(\mathrm{R}_{1}, \mathrm{R}_{2}, \mathrm{~S}_{1}, \mathrm{~S}_{1}^{\prime}, \mathrm{S}_{2}, \mathrm{~S}_{2}^{\prime}\right)$ were $\sigma$. Note that $\sigma$ is never rejected in Step 4 and passes the Swap-Test in Step 5 with certainty, and the state is not changed at all in these two steps. Hence, if the state in $\left(R_{1}, R_{2}, S_{1}, S_{1}^{\prime}, S_{2}, S_{2}^{\prime}\right)$ were $\sigma$ when entering Step 4, the protocol should result in rejection with probability at least $\frac{1}{16}-\frac{\pi}{2} \delta^{\frac{1}{2}}$, as claimed.

We next show the following proposition, which is more general than Proposition 22, but still is a special case of Proposition 21

Proposition 24. Let $\mathcal{W}$ be the two-dimensional space spanned by $\left|\Phi^{-}\right\rangle$and $\left|\Psi^{+}\right\rangle$. When entering Step 4 of the protocol described in Figure 6 suppose that the state in $\left(\mathrm{R}_{1}, \mathrm{R}_{2}, \mathrm{~S}_{1}, \mathrm{~S}_{1}^{\prime}, \mathrm{S}_{2}, \mathrm{~S}_{2}^{\prime}\right)$ were of the form $(|0\rangle\langle 0|)^{\otimes 2} \otimes \tau$ where $\tau=\sum_{j} \mu_{j} \xi_{j}{ }^{\otimes 2}$ for some two-qubit states $\xi_{j} \in \mathbf{D}(\mathcal{W})$ and real numbers $\mu_{j} \in[0,1]$ satisfying $\sum_{j} \mu_{j}=1$, such that the reduced state of $\tau$ in $\left(\mathrm{S}_{1}, \mathrm{~S}_{2}\right)$ has trace distance at most $\delta$ to the two-qubit totally mixed state $(I / 2)^{\otimes 2}$ for some positive $\delta$ satisfying $\frac{1}{16}-10 \delta^{\frac{1}{4}}>0$. Then the protocol should result in rejection with probability at least $\min \left\{2 \delta, \frac{1}{16}-10 \delta^{\frac{1}{4}}\right\}$.

Proof. Let $\sigma=(|0\rangle\langle 0|)^{\otimes 2} \otimes \tau$. Note that $\sigma$ is never rejected in Step 4, and the state is not changed at all in this step.

Fix a constant $\gamma_{1} \in(0,1)$, and let $S$ be the set of indices $j$ defined by

$$
S=\left\{j: \operatorname{tr} \xi_{j}^{2} \geq 1-\gamma_{1}\right\}
$$

Notice that the inequality $\operatorname{tr}_{j}{ }^{2} \geq 1-\gamma_{1}$ implies that the maximum eigenvalue of the Hermitian matrix $\xi_{j}$ is at least $1-\gamma_{1}$, and thus, for each $j \in S$, there exist a two-qubit pure state $\left|\psi_{j}\right\rangle \in \mathcal{W}$, a two-qubit state $\nu_{j} \in \mathbf{D}(\mathcal{W})$, and a real number $\lambda_{j} \in\left[1-\gamma_{1}, 1\right]$ such that

$$
\xi_{j}=\lambda_{j}\left|\psi_{j}\right\rangle\left\langle\psi_{j}\right|+\left(1-\lambda_{j}\right) \nu_{j}
$$

This implies that

$$
\| \xi_{j}-\left|\psi_{j}\right\rangle\left\langle\psi_{j}\left|\left\|_{\mathrm{tr}}=\right\| \lambda_{j}\right| \psi_{j}\right\rangle\left\langle\psi_{j}\left|+\left(1-\lambda_{j}\right) \nu_{j}-\right| \psi_{j}\right\rangle\left\langle\psi_{j}\left|\left\|_{\mathrm{tr}}=\left(1-\lambda_{j}\right)\right\| \nu_{j}-\right| \psi_{j}\right\rangle\left\langle\psi_{j}\right| \|_{\mathrm{tr}}
$$


which further implies that

$$
D\left(\xi_{j},\left|\psi_{j}\right\rangle\left\langle\psi_{j}\right|\right) \leq\left(1-\lambda_{j}\right) D\left(\nu_{j},\left|\psi_{j}\right\rangle\left\langle\psi_{j}\right|\right) \leq 1-\lambda_{j} \leq \gamma_{1} .
$$

Fix another constant $\gamma_{2} \in(0,1)$.

If $\sum_{j \in S} \mu_{j}<1-\gamma_{2}$, the SWAP TEST in Step 5 results in rejection with probability greater than $\frac{1}{2} \gamma_{1} \gamma_{2}$.

On the other hand, if $\sum_{j \in S} \mu_{j} \geq 1-\gamma_{2}$, the state $\sigma$ has trace distance at most $2 \gamma_{1}+\gamma_{2}$ to the state $\sigma^{\prime}=(|0\rangle\langle 0|)^{\otimes 2} \otimes \tau^{\prime}$, where

$$
\tau^{\prime}=\frac{1}{\sum_{j \in S} \mu_{j}} \sum_{j \in S} \mu_{j}\left(\left|\psi_{j}\right\rangle\left\langle\psi_{j}\right|\right)^{\otimes 2}
$$

and the reduced state of $\tau^{\prime}$ in $\left(\mathrm{S}_{1}, \mathrm{~S}_{2}\right)$ has trace distance at most $\delta+2 \gamma_{1}+\gamma_{2}$ to $(I / 2)^{\otimes 2}$.

Indeed,

$$
\begin{aligned}
\left\|\tau-\tau^{\prime}\right\|_{\mathrm{tr}}= & \left\|\sum_{j} \mu_{j} \xi_{j}^{\otimes 2}-\tau^{\prime}\right\|_{\mathrm{tr}} \\
\leq & \left\|\sum_{j} \mu_{j} \xi_{j}^{\otimes 2}-\left(\sum_{j \in S} \mu_{j}\left(\left|\psi_{j}\right\rangle\left\langle\psi_{j}\right|\right)^{\otimes 2}+\sum_{j \notin S} \mu_{j} \xi_{j}^{\otimes 2}\right)\right\|_{\mathrm{tr}} \\
& +\left\|\left(\sum_{j \in S} \mu_{j}\left(\left|\psi_{j}\right\rangle\left\langle\psi_{j}\right|\right)^{\otimes 2}+\sum_{j \notin S} \mu_{j} \xi_{j}^{\otimes 2}\right)-\tau^{\prime}\right\|_{\mathrm{tr}} \\
\leq & \sum_{j \in S} \mu_{j}\left\|\xi_{j}^{\otimes 2}-\left(\left|\psi_{j}\right\rangle\left\langle\psi_{j}\right|\right)^{\otimes 2}\right\|_{\mathrm{tr}}+\left\|\sum_{j \notin S} \mu_{j} \xi_{j}^{\otimes 2}-\left(\frac{1}{\sum_{j \in S} \mu_{j}}-1\right) \sum_{j \in S} \mu_{j}\left(\left|\psi_{j}\right\rangle\left\langle\psi_{j}\right|\right)^{\otimes 2}\right\|_{\mathrm{tr}} \\
\leq & \sum_{j \in S} \mu_{j}\left(\| \xi_{j}^{\otimes 2}-\left|\psi_{j}\right\rangle\left\langle\psi_{j}\left|\otimes \xi_{j}\left\|_{\mathrm{tr}}+\right\|\right| \psi_{j}\right\rangle\left\langle\psi_{j}\right| \otimes \xi_{j}-\left(\left|\psi_{j}\right\rangle\left\langle\psi_{j}\right|\right)^{\otimes 2} \|_{\mathrm{tr}}\right) \\
& +\left(1-\sum_{j \in S} \mu_{j}\right)\left\|\frac{1}{\sum_{j \notin S} \mu_{j}} \sum_{j \notin S} \mu_{j} \xi_{j}^{\otimes 2}-\tau^{\prime}\right\|_{\mathrm{tr}} \\
\leq & 2 \sum_{j \in S} \mu_{j} \| \xi_{j}-\left|\psi_{j}\right\rangle\left\langle\psi_{j}\right|\left\|_{\mathrm{tr}}+\left(1-\sum_{j \in S} \mu_{j}\right)\right\|_{\sum_{j \notin S} \mu_{j}} \sum_{j \notin S} \mu_{j} \xi_{j}^{\otimes 2}-\tau^{\prime} \|_{\mathrm{tr}},
\end{aligned}
$$

and thus,

$$
\begin{aligned}
D\left(\sigma, \sigma^{\prime}\right) & =D\left(\tau, \tau^{\prime}\right) \\
& \leq 2 \sum_{j \in S} \mu_{j} D\left(\xi_{j},\left|\psi_{j}\right\rangle\left\langle\psi_{j}\right|\right)+\left(1-\sum_{j \in S} \mu_{j}\right) D\left(\frac{1}{\sum_{j \notin S} \mu_{j}} \sum_{j \notin S} \mu_{j} \xi_{j}^{\otimes 2}, \tau^{\prime}\right) \\
& \leq 2 \gamma_{1}+\gamma_{2} .
\end{aligned}
$$

As the reduced state of $\tau$ in $\left(\mathrm{S}_{1}, \mathrm{~S}_{2}\right)$ has trace distance at most $\delta$ to $(I / 2)^{\otimes 2}$, it follows that the reduced state of $\tau^{\prime}$ in $\left(\mathrm{S}_{1}, \mathrm{~S}_{2}\right)$ has trace distance at most $\delta+2 \gamma_{1}+\gamma_{2}$ to $(I / 2)^{\otimes 2}$. Now from Proposition 22, the protocol should result in rejection with probability at least $\frac{1}{16}-\frac{\pi}{2}\left(\delta+2 \gamma_{1}+\gamma_{2}\right)^{\frac{1}{2}}$ if the state in $\left(\mathrm{R}_{1}, \mathrm{R}_{2}, \mathrm{~S}_{1}, \mathrm{~S}_{1}^{\prime}, \mathrm{S}_{2}, \mathrm{~S}_{2}^{\prime}\right)$ were $\sigma^{\prime}$ when entering Step 4. Hence, from Lemma 6 the protocol should result in rejection with probability at least $\frac{1}{16}-2 \gamma_{1}-\gamma_{2}-\frac{\pi}{2}\left(\delta+2 \gamma_{1}+\gamma_{2}\right)^{\frac{1}{2}}$ if the state in the registers $\left(\mathrm{R}_{1}, \mathrm{R}_{2}, \mathrm{~S}_{1}, \mathrm{~S}_{1}^{\prime}, \mathrm{S}_{2}, \mathrm{~S}_{2}^{\prime}\right)$ were $\sigma$ when entering Step 4.

Overall, the protocol should result in rejection with probability at least

$$
\min \left\{\frac{1}{2} \gamma_{1} \gamma_{2}, \frac{1}{16}-2 \gamma_{1}-\gamma_{2}-\frac{\pi}{2}\left(\delta+2 \gamma_{1}+\gamma_{2}\right)^{\frac{1}{2}}\right\}
$$


if the state in $\left(\mathrm{R}_{1}, \mathrm{R}_{2}, \mathrm{~S}_{1}, \mathrm{~S}_{1}^{\prime}, \mathrm{S}_{2}, \mathrm{~S}_{2}^{\prime}\right)$ were $\sigma$ when entering Step 4. Taking $\gamma_{1}=\sqrt{2} \delta^{\frac{1}{2}}$ and $\gamma_{2}=2 \sqrt{2} \delta^{\frac{1}{2}}$, this is at least

$$
\min \left\{2 \delta, \frac{1}{16}-4 \sqrt{2} \delta^{\frac{1}{2}}-\frac{\pi}{2}\left(\delta+4 \sqrt{2} \delta^{\frac{1}{2}}\right)^{\frac{1}{2}}\right\} \geq \min \left\{2 \delta, \frac{1}{16}-4 \sqrt{2} \delta^{\frac{1}{2}}-\frac{\pi}{2} \sqrt{7} \delta^{\frac{1}{4}}\right\} \geq \min \left\{2 \delta, \frac{1}{16}-10 \delta^{\frac{1}{4}}\right\},
$$

which completes the proof.

Now we are ready to prove Proposition 21 .

Proof of Proposition 21] Let $\sigma=(|0\rangle\langle 0|)^{\otimes 2} \otimes \tau$. Let $\mathcal{W}$ be the two-dimensional space spanned by $\left|\Phi^{-}\right\rangle$and $\left|\Psi^{+}\right\rangle$, and let $\Pi_{\mathcal{W}}=\left|\Phi^{-}\right\rangle\left\langle\Phi^{-}|+| \Psi^{+}\right\rangle\left\langle\Psi^{+}\right|$be the projection onto $\mathcal{W}$.

Fix a constant $\gamma \in(0,1)$.

If $\operatorname{tr} \Pi_{\mathcal{W}}^{\otimes 2} \tau<1-\gamma$, Step 4 results in rejection with probability greater than $\gamma$.

On the other hand, if $\operatorname{tr} \Pi_{\mathcal{W}}^{\otimes 2} \tau \geq 1-\gamma$, we claim that the state $\sigma$ has trace distance at most $\sqrt{\gamma}$ to the state $\sigma^{\prime}=(|0\rangle\langle 0|)^{\otimes 2} \otimes \tau^{\prime}$, where

$$
\tau^{\prime}=\sum_{j} \mu_{j}^{\prime} \xi_{j}^{\prime \otimes 2}
$$

with

$$
\mu_{j}^{\prime}=\frac{1}{\operatorname{tr}_{\mathcal{W}}^{\otimes 2} \tau}\left(\operatorname{tr} \Pi_{\mathcal{W}} \xi_{j}\right)^{2} \mu_{j}, \quad \xi_{j}^{\prime}=\frac{1}{\operatorname{tr} \Pi_{\mathcal{W}} \xi_{j}} \Pi_{\mathcal{W}} \xi_{j} \Pi_{\mathcal{W}}
$$

for each $j$, and the reduced state of $\tau^{\prime}$ in $\left(\mathrm{S}_{1}, \mathrm{~S}_{2}\right)$ has trace distance at most $\gamma$ to $(I / 2)^{\otimes 2}$. Note that $\mu_{j}^{\prime} \in[0,1]$ and $\xi_{j}^{\prime} \in \mathbf{D}(\mathcal{W})$ for each $j$, and $\sum_{j} \mu_{j}^{\prime}=1$.

Let $\mathcal{S}$ be the $2^{4}$-dimensional Hilbert space $\mathbb{C}\left(\Sigma^{4}\right)$ associated with the quantum register $\left(\mathrm{S}_{1}, \mathrm{~S}_{1}^{\prime}, \mathrm{S}_{2}, \mathrm{~S}_{2}^{\prime}\right)$ and let $\mathcal{T}$ be another $2^{4}$-dimensional Hilbert space $\mathbb{C}\left(\Sigma^{4}\right)$. Consider any purification $|\psi\rangle \in \mathcal{S} \otimes \mathcal{T}$ of $\tau \in \mathbf{D}(\mathcal{S})$, and define an eight-qubit pure state $\left|\psi^{\prime}\right\rangle \in \mathcal{S} \otimes \mathcal{T}$ by

$$
\left|\psi^{\prime}\right\rangle=\frac{1}{\|\left(\Pi_{\mathcal{W}}^{\otimes 2} \otimes I_{\mathcal{T}}\right)|\psi\rangle \|}\left(\Pi_{\mathcal{W}}^{\otimes 2} \otimes I_{\mathcal{T}}\right)|\psi\rangle .
$$

Then, $\left|\psi^{\prime}\right\rangle$ is a purification of $\tau^{\prime}$, since

$$
\begin{aligned}
\operatorname{tr}_{\mathcal{T}}\left|\psi^{\prime}\right\rangle\left\langle\psi^{\prime}\left|=\frac{1}{\|\left(\Pi_{\mathcal{W}}^{\otimes 2} \otimes I_{\mathcal{T}}\right)|\psi\rangle \|^{2}} \operatorname{tr}_{\mathcal{T}}\left(\Pi_{\mathcal{W}}^{\otimes 2} \otimes I_{\mathcal{T}}\right)\right| \psi\right\rangle\langle\psi|\left(\Pi_{\mathcal{W}}^{\otimes 2} \otimes I_{\mathcal{T}}\right) \\
=\frac{1}{\operatorname{tr} \Pi_{\mathcal{W}}^{\otimes 2} \operatorname{tr}|\psi\rangle\langle\psi|} \Pi_{\mathcal{W}}^{\otimes 2}\left(\operatorname{tr}_{\mathcal{T}}|\psi\rangle\langle\psi|\right) \Pi_{\mathcal{W}}^{\otimes 2}=\frac{1}{\operatorname{tr} \Pi_{\mathcal{W}}^{\otimes 2} \tau} \Pi_{\mathcal{W}}^{\otimes 2} \tau \Pi_{\mathcal{W}}^{\otimes 2}=\tau^{\prime},
\end{aligned}
$$

where the last equality follows from the fact that

$$
\tau^{\prime}=\sum_{j} \frac{1}{\operatorname{tr} \Pi_{\mathcal{W}}^{\otimes 2} \tau}\left(\operatorname{tr} \Pi_{\mathcal{W}} \xi_{j}\right)^{2} \mu_{j}\left(\frac{1}{\operatorname{tr} \Pi_{\mathcal{W}} \xi_{j}} \Pi_{\mathcal{W}} \xi_{j} \Pi_{\mathcal{W}}\right)^{\otimes 2}=\frac{1}{\operatorname{tr} \Pi_{\mathcal{W}}^{\otimes 2} \tau} \sum_{j} \mu_{j}\left(\Pi_{\mathcal{W}} \xi_{j} \Pi_{\mathcal{W}}\right)^{\otimes 2}=\frac{1}{\operatorname{tr} \Pi_{\mathcal{W}}^{\otimes 2} \tau} \Pi_{\mathcal{W}}^{\otimes 2} \tau \Pi_{\mathcal{W}}^{\otimes 2} .
$$

Therefore, by using the fact that $D\left(|\psi\rangle\left\langle\psi|,| \psi^{\prime}\right\rangle\left\langle\psi^{\prime}\right|\right)=\sqrt{1-\left|\left\langle\psi \mid \psi^{\prime}\right\rangle\right|^{2}}$ holds for any pure states $|\psi\rangle$ and $\left|\psi^{\prime}\right\rangle$ (which is ensured by calculating eigenvalues of the Hermitian matrix $|\psi\rangle\left\langle\psi|-| \psi^{\prime}\right\rangle\left\langle\psi^{\prime}\right|$ ),

$$
\begin{aligned}
& D\left(\sigma, \sigma^{\prime}\right)=D\left(\tau, \tau^{\prime}\right) \\
& \leq D\left(|\psi\rangle\left\langle\psi|,| \psi^{\prime}\right\rangle\left\langle\psi^{\prime}\right|\right)=\sqrt{1-\left|\left\langle\psi \mid \psi^{\prime}\right\rangle\right|^{2}}=\sqrt{1-\|\left(\Pi_{\mathcal{W}}^{\otimes 2} \otimes I_{\mathcal{T}}\right)|\psi\rangle \|^{2}}=\sqrt{1-\operatorname{tr} \Pi_{\mathcal{W}}^{\otimes 2} \tau} \leq \sqrt{\gamma} .
\end{aligned}
$$

As the reduced state of $\tau$ in $\left(\mathrm{S}_{1}, \mathrm{~S}_{2}\right)$ has trace distance at most $\delta$ to $(I / 2)^{\otimes 2}$, it follows that the reduced state of $\tau^{\prime}$ in $\left(\mathrm{S}_{1}, \mathrm{~S}_{2}\right)$ has trace distance at most $\delta+\sqrt{\gamma}$ to $(I / 2)^{\otimes 2}$. Now from Proposition 24, the protocol should result 
in rejection with probability at least $\min \left\{2(\delta+\sqrt{\gamma}), \frac{1}{16}-10(\delta+\sqrt{\gamma})^{\frac{1}{4}}\right\}$ if the state in $\left(\mathrm{R}_{1}, \mathrm{R}_{2}, \mathrm{~S}_{1}, \mathrm{~S}_{1}^{\prime}, \mathrm{S}_{2}, \mathrm{~S}_{2}^{\prime}\right)$ were $\sigma^{\prime}$ when entering Step 4. Hence, Lemma 6 implies that the protocol should result in rejection with probability at least $\min \left\{2(\delta+\sqrt{\gamma})-\sqrt{\gamma}, \frac{1}{16}-\sqrt{\gamma}-10(\delta+\sqrt{\gamma})^{\frac{1}{4}}\right\}$ if the state in $\left(\mathrm{R}_{1}, \mathrm{R}_{2}, \mathrm{~S}_{1}, \mathrm{~S}_{1}^{\prime}, \mathrm{S}_{2}, \mathrm{~S}_{2}^{\prime}\right)$ were $\sigma$ when entering Step 4.

Overall, the protocol should result in rejection with probability at least

$$
\min \left\{\gamma, 2(\delta+\sqrt{\gamma})-\sqrt{\gamma}, \frac{1}{16}-\sqrt{\gamma}-10(\delta+\sqrt{\gamma})^{\frac{1}{4}}\right\}
$$

if the state in $\left(R_{1}, R_{2}, S_{1}, S_{1}^{\prime}, S_{2}, S_{2}^{\prime}\right)$ were $\sigma$ when entering Step 4. Taking $\gamma=2 \delta$, this is at least

$\min \left\{2 \delta, 2 \delta+(2 \delta)^{\frac{1}{2}}, \frac{1}{16}-(2 \delta)^{\frac{1}{2}}-10\left[\delta+(2 \delta)^{\frac{1}{2}}\right]^{\frac{1}{4}}\right\} \geq \min \left\{2 \delta, \frac{1}{16}-(2 \delta)^{\frac{1}{2}}-10\left(3 \delta^{\frac{1}{2}}\right)^{\frac{1}{4}}\right\} \geq \min \left\{2 \delta, \frac{1}{16}-15 \delta^{\frac{1}{8}}\right\}$, which completes the proof.

\section{$7 \quad \mathrm{QIP}(m) \subseteq \mathrm{QIP}_{1}(m+1)$}

Now we show that any $m$-message QIP system with two-sided bounded error can be converted into an $(m+1)$ message QIP system with one-sided error of perfect completeness, for any $m \geq 2$.

Theorem 25. For any polynomially bounded function $m: \mathbb{Z}^{+} \rightarrow \mathbb{N}$ and polynomial-time computable functions $c, s: \mathbb{Z}^{+} \rightarrow[0,1]$ satisfying $m \geq 2$ and $c-s \geq 1 /$ for some polynomially bounded function $p: \mathbb{Z}^{+} \rightarrow \mathbb{N}$,

$$
\mathrm{QIP}(m, c, s) \subseteq \mathrm{QIP}\left(m+1,1,1-\frac{(c-s)^{2}}{16}\right) .
$$

If $m$ is an odd-valued function whose values are at least three, we can show a stronger statement that any $m$-message QIP system with two-sided bounded error can be converted into another $m$-message QIP system with one-sided error of perfect completeness.

Theorem 26. For any polynomially bounded odd-valued function $m: \mathbb{Z}^{+} \rightarrow 2 \mathbb{N}+1$ and polynomial-time computable functions $c, s: \mathbb{Z}^{+} \rightarrow[0,1]$ satisfying $m \geq 3$ and $c-s \geq 1 / p$ for some polynomially bounded function $p: \mathbb{Z}^{+} \rightarrow \mathbb{N}$,

$$
\mathrm{QIP}(m, c, s) \subseteq \operatorname{QIP}\left(m, 1,1-\frac{(c-s)^{2}}{16}\right) .
$$

Remark. In fact, in Theorems 25 and 26, it is sufficient for the claims that the functions $c$ and $s$ satisfy $c-s \geq 2^{-p}$ for some polynomially bounded function $p: \mathbb{Z}^{+} \rightarrow \mathbb{N}$.

With the perfect parallel repetition theorem for general quantum interactive proofs [Gut09], the following corollaries immediately follow.

Corollary 27. For any polynomially bounded functions $m, p: \mathbb{Z}^{+} \rightarrow \mathbb{N}$ and polynomial-time computable functions $c, s: \mathbb{Z}^{+} \rightarrow[0,1]$ satisfying $m \geq 2$ and $c-s \geq 1 / q$ for some polynomially bounded function $q: \mathbb{Z}^{+} \rightarrow \mathbb{N}$,

$$
\mathrm{QIP}(m, c, s) \subseteq \mathrm{QIP}\left(m+1,1,2^{-p}\right) .
$$

Corollary 28. For any polynomially bounded odd-valued function $m: \mathbb{Z}^{+} \rightarrow 2 \mathbb{N}+1$, polynomially bounded function $p: \mathbb{Z}^{+} \rightarrow \mathbb{N}$, and polynomial-time computable functions $c, s: \mathbb{Z}^{+} \rightarrow[0,1]$ satisfying $m \geq 3$ and $c-s \geq 1 / q$ for some polynomially bounded function $q: \mathbb{Z}^{+} \rightarrow \mathbb{N}$,

$$
\mathrm{QIP}(m, c, s) \subseteq \operatorname{QIP}\left(m, 1,2^{-p}\right)
$$




\section{Modified Reflection Procedure}

1. Receive a quantum register Q. Flip a fair coin, and proceed to the REFLECTION TEST in Step 2 if it results in "Heads", and proceed to the INVERTIBILITY TEST in Step 3 if it results in "Tails".

2. (REFLECTION TEST)

Perform the following:

2.1 Perform a phase-flip (i.e., multiply -1 in phase) if the state in $\mathrm{Q}$ belongs to the subspace corresponding to the projection $\Pi_{0}$.

2.2 Apply $U^{\dagger}$ to $Q$.

2.3 Reject if the state in $Q$ belongs to the subspace corresponding to the projection $\Delta_{0}$, and accept otherwise.

3. (INVERTibiLity Test)

Perform the following:

3.1 Apply $U^{\dagger}$ to Q.

3.2 Accept if the state in $\mathrm{Q}$ belongs to the subspace corresponding to $\Delta_{0}$, and reject otherwise.

Figure 7: The Modified ReFlection Procedure.

\subsection{Modified Reflection Procedure}

The Reflection Procedure in Section 5 involves one application of $U$ and one application of $U^{\dagger}$. Here we modify the procedure so that it involves one application of $U^{\dagger}$ only (and no application of $U$ is required).

To do this, one expects to receive a state just after Step 1 of the REFLECTION PROCEDURE, and performs two tests, called REFLECTION TEST and INVERTIBILITY TEST, respectively, with equal probability without revealing which test the prover is undergoing. In the REFLECTION TEST, we simply perform Steps $2-4$ of the REFLECTION Procedure to finish the simulation of it, whereas in the INVERTIBILITY TEST, we apply $U^{\dagger}$ without performing the phase-flip to check that the state received was a legal state that can appear just after Step 1 of the REFLECTION PROCEDURE. The idea of making use of the INVERTIBILITY TEST has originally appeared in Ref. [KKMV09] when achieving perfect completeness in quantum multi-prover interactive proofs. From another viewpoint, the modification here may be considered as applying the "halving technique" in Ref. [KKMV09] to the REFLECTION PROCEDURE, the technique originally used to reduce the number of turns by (almost) half in quantum multi-prover interactive proofs. We will take this view when analyzing the soundness of this procedure in Proposition 30 below. The procedure is summarized in Figure 7

Proposition 29. Suppose that the Hermitian operator $M=\Delta_{0} U^{\dagger} \Pi_{0} U \Delta_{0}$ has an eigenvalue $1 / 2$. Then there exists a quantum state given in Step 1 of the MODIFIED REFLECTION PROCEDURE such that the procedure results in acceptance with certainty.

Proof. The proof is almost straightforward. Let $\left|\psi^{*}\right\rangle$ be an eigenvector of $M$ corresponding to its eigenvalue $1 / 2$, and consider the case where the state $U\left|\psi^{*}\right\rangle$ is received in Q in Step 1.

If the REFLECTION TEST is performed, this essentially simulates the original REFLECTION PROCEDURE with its received state being $\left|\psi^{*}\right\rangle$. As in the case of Proposition 17, the procedure results in acceptance with certainty in this case.

On the other hand, if the INVERTIBILITy TEST is performed, this produces the state $U^{\dagger} U\left|\psi^{*}\right\rangle=\left|\psi^{*}\right\rangle$ when 
entering Step 3.2. As $\left|\psi^{*}\right\rangle$ is an eigenvector of $M$ with its corresponding eigenvalue $1 / 2$, it holds that

$$
\Delta_{0}\left|\psi^{*}\right\rangle=2 \Delta_{0} M\left|\psi^{*}\right\rangle=2 M\left|\psi^{*}\right\rangle=\left|\psi^{*}\right\rangle,
$$

and thus, Step 3.2 results in acceptance with certainty.

Hence, given the state $U\left|\psi^{*}\right\rangle$ in Step 1, the procedure results in acceptance with certainty, and the claim follows.

Proposition 30. For any $\varepsilon \in\left(0, \frac{1}{2}\right]$, suppose that none of the eigenvalues of the Hermitian operator $M=\Delta_{0} U^{\dagger} \Pi_{0} U \Delta_{0}$ is in the interval $\left(\frac{1}{2}-\varepsilon, \frac{1}{2}+\varepsilon\right)$. Then, for any quantum state given in Step 1 of the MODIFIED REFLECTION PROCEDURE, the procedure results in rejection with probability at least $\varepsilon^{2}$.

Proof. The proof is similar to the proofs of Lemmas 4.1 and 5.1 in Ref. [KKMV09]. Let $|\psi\rangle$ be any state received in $\mathrm{Q}$ in Step 1. Denote the unitary transformation $U^{\dagger}\left(-\Pi_{0}+\Pi_{1}\right)$ by $V$, and let

$$
|\alpha\rangle=\frac{\Delta_{1} V|\psi\rangle}{\| \Delta_{1} V|\psi\rangle \|}, \quad|\beta\rangle=\frac{\Delta_{0} U^{\dagger}|\psi\rangle}{\| \Delta_{0} U^{\dagger}|\psi\rangle \|}
$$

Then

$$
\| \Delta_{1} V|\psi\rangle \|=\frac{1}{\| \Delta_{1} V|\psi\rangle \|}\left|\left\langle\psi\left|V^{\dagger} \Delta_{1} V\right| \psi\right\rangle\right|=F\left(|\alpha\rangle\langle\alpha|, V| \psi\rangle\langle\psi| V^{\dagger}\right)=F\left(V^{\dagger}|\alpha\rangle\langle\alpha|V,| \psi\rangle\langle\psi|\right),
$$

and thus, the probability $p_{1}$ of acceptance when the REFLECTION TEST is performed is given by

$$
p_{1}=F\left(V^{\dagger}|\alpha\rangle\langle\alpha|V,| \psi\rangle\langle\psi|\right)^{2} .
$$

Similarly, the probability $p_{2}$ of acceptance when the INVERTIBILITY TEST is performed is given by

$$
p_{2}=F\left(U|\beta\rangle\left\langle\beta\left|U^{\dagger},\right| \psi\right\rangle\langle\psi|\right)^{2} .
$$

Hence, the probability $p_{\text {acc }}$ of acceptance when the received state in Step 1 was $|\psi\rangle$ is given by

$$
p_{\text {acc }}=\frac{1}{2}\left(p_{1}+p_{2}\right)=\frac{1}{2}\left(F\left(V^{\dagger}|\alpha\rangle\langle\alpha|V,| \psi\rangle\langle\psi|\right)^{2}+F\left(U|\beta\rangle\left\langle\beta\left|U^{\dagger},\right| \psi\right\rangle\langle\psi|\right)^{2}\right) .
$$

It follows from Lemma 7 that

$$
p_{\text {acc }} \leq \frac{1}{2}\left(1+F\left(V^{\dagger}|\alpha\rangle\langle\alpha|V, U| \beta\rangle\langle\beta| U^{\dagger}\right)\right)=\frac{1}{2}\left(1+F\left(|\alpha\rangle\langle\alpha|, V U| \beta\rangle\langle\beta| U^{\dagger} V^{\dagger}\right)\right) .
$$

Now notice that $|\beta\rangle$ is a state in $\mathcal{X}_{0}$, and thus,

$$
\| \Delta_{1} V U|\beta\rangle \|^{2} \leq 1-4 \varepsilon^{2},
$$

since $\| \Delta_{0} V U|\beta\rangle \|^{2} \geq 4 \varepsilon^{2}$ from the analysis on the REFLECTION PROCEDURE in the proof of Proposition 18 Hence, using $\Delta_{1}|\alpha\rangle=|\alpha\rangle$,

$$
F\left(|\alpha\rangle\langle\alpha|, V U| \beta\rangle\langle\beta| U^{\dagger} V^{\dagger}\right)=|\langle\alpha|V U| \beta\rangle|=\left|\left\langle\alpha\left|\Delta_{1} V U\right| \beta\right\rangle\right| \leq \| \Delta_{1} V U|\beta\rangle \| \leq \sqrt{1-4 \varepsilon^{2}},
$$

and thus,

$$
p_{\text {acc }} \leq \frac{1}{2}+\frac{\sqrt{1-4 \varepsilon^{2}}}{2} \leq \frac{1}{2}+\frac{1-2 \varepsilon^{2}}{2}=1-\varepsilon^{2}
$$

Therefore, the procedure results in rejection with probability at least $\varepsilon^{2}$, as claimed. 


\subsection{Perfectly Rewindable QIPs}

Here we introduce the notion of perfectly rewindable QIP systems. The concept of perfectly rewindable systems was originally introduced for quantum multi-prover interactive proofs in Ref. [KKMV09], and the notion here is the single-prover version of it as a special case.

Definition 31. Given a polynomially bounded function $m: \mathbb{Z}^{+} \rightarrow \mathbb{N}$ and a function $s: \mathbb{Z}^{+} \rightarrow[0,1]$ satisfying $s<\frac{1}{2}$, a promise problem $A=\left\{A_{\text {yes }}, A_{\text {no }}\right\}$ has a perfectly rewindable $m$-message quantum interactive proof system with soundness $s$ iff there exists an $m$-message polynomial-time quantum verifier $V$ such that, for every input $x$ :

(Perfect Rewindability) if $x \in A_{\text {yes }}$, there exists an $m$-message quantum prover $P$ such that the maximum probability that $V$ accepts $x$ when communicating with $P$ is exactly $1 / 2$, where the maximum is taken over all possible initial states $\rho_{x}$ of $P$,

(Soundness) if $x \in A_{\text {no }}$, for any $m$-message quantum prover $P^{\prime}$ and any initial state $\rho_{x}^{\prime}$ of $P^{\prime}$ prepared, $V$ accepts $x$ with probability at most $s(|x|)$.

Note that in the perfect rewindability property we first fix the transformations of the prover, and then maximize over all legal initial states, which hence have a fixed dimension. We first show how to modify any general QIP system to a perfectly rewindable one without changing the number of messages.

Lemma 32. Let $m: \mathbb{Z}^{+} \rightarrow \mathbb{N}$ be a polynomially bounded function and let $c, s: \mathbb{Z}^{+} \rightarrow[0,1]$ be polynomial-time computable functions satisfying $c-s \geq 1 / p$ for some polynomially bounded function $p: \mathbb{Z}^{+} \rightarrow \mathbb{N}$. Then, any promise problem $A=\left(A_{\mathrm{yes}}, A_{\mathrm{no}}\right)$ in $\mathrm{QIP}(m, c, s)$ has a perfectly rewindable m-message quantum interactive proof system with soundness $\frac{1}{2}-\frac{c-s}{4}$.

Proof. Let $A=\left(A_{\text {yes }}, A_{\text {no }}\right)$ be a problem in $\operatorname{QIP}(m, c, s)$ and let $V$ be the corresponding $m$-message quantum verifier. We first modify $V$ to obtain another $m$-message quantum verifier $V^{\prime}$ that witnesses the inclusion $A \in \operatorname{QIP}\left(m, \frac{1}{2}+\frac{c-s}{4}, \frac{1}{2}-\frac{c-s}{4}\right)$. This can be done via a standard technique as follows. Fix an input $x$. The new verifier $V^{\prime}$ behaves in a manner exactly same as $V$, except for the acceptance condition. If $c(|x|)+s(|x|) \geq 1$, $V^{\prime}$ accepts with probability $\frac{1}{c(|x|)+s(|x|)}$ when the final state in the system would make $V$ accept (and reject otherwise). Thus, $V^{\prime}$ accepts $x \in A_{\text {yes }}$ with probability at least $\frac{c(|x|)}{c(|x|)+s(|x|)}=\frac{1}{2}\left(1+\frac{c(|x|)-s(|x|)}{c(|x|)+s(|x|)}\right)$, while accepts $x \in A_{\text {no }}$ with probability at most $\frac{s(|x|)}{c(|x|)+s(|x|)}=\frac{1}{2}\left(1-\frac{c(|x|)-s(|x|)}{c(|x|)+s(|x|)}\right)$. Similarly, if $c(|x|)+s(|x|)<1$, letting $\varepsilon(|x|)=1-c(|x|)$ and $\delta(|x|)=1-s(|x|), V^{\prime}$ rejects with probability $\frac{1}{\varepsilon(|x|)+\delta(|x|)}=\frac{1}{2-c(|x|)-s(|x|)}$ when the final state in the system would make $V$ reject (and accept otherwise). Thus, $V^{\prime}$ rejects $x \in A_{\text {yes }}$ with probability at most $\frac{\varepsilon(|x|)}{\varepsilon(|x|)+\delta(|x|)}=\frac{1}{2}\left(1-\frac{\delta(|x|)-\varepsilon(|x|)}{\varepsilon(|x|)+\delta(|x|)}\right)=\frac{1}{2}\left(1-\frac{c(|x|)-s(|x|)}{2-c(|x|)-s(|x|)}\right)$, while $V^{\prime}$ rejects $x \in A_{\text {no }}$ with probability at least $\frac{\delta(|x|)}{\varepsilon(|x|)+\delta(|x|)}=\frac{1}{2}\left(1+\frac{\delta(|x|)-\varepsilon(|x|)}{\varepsilon(|x|)+\delta(|x|)}\right)=\frac{1}{2}\left(1+\frac{c(|x|)-s(|x|)}{2-c(|x|)-s(|x|)}\right)$. Taking it into account that, with a given finite-size gate set available for the verifier, it may not be possible to accept with probability exactly $\frac{1}{c(|x|)+s(|x|)}$ in the case $c(|x|)+s(|x|) \geq 1$, or to reject with probability exactly $\frac{1}{\varepsilon(|x|)+\delta(|x|)}=\frac{1}{2-c(|x|)-s(|x|)}$ in the case $c(|x|)+s(|x|)<1$, we actually consider another verifier $V^{\prime \prime}$ who approximately performs the transformations of $V^{\prime}$ with sufficient accuracy, where the transformations of $V^{\prime \prime}$ are exactly implementable with the given finite-size gate set available for the verifier. As both $c(|x|)+s(|x|)$ and $2-c(|x|)-s(|x|)$ are at most $2-\frac{1}{p}$, the bounds obtained above are sufficient to claim that the $m$-message system with the verifier $V^{\prime \prime}$ has completeness $\frac{1}{2}+\frac{c-s}{4}$ and soundness $\frac{1}{2}-\frac{c-s}{4}$.

The rest of the proof is essentially the same as the proof of Lemma 3.2 in Ref. [KKMV09]. We further modify $V^{\prime \prime}$ to construct another $m$-message quantum verifier $W$ for a perfectly rewindable proof system for $A$. The new verifier $W$ prepares a single-qubit register $\mathrm{B}$ in addition to the register $\mathrm{V}$ which corresponds to the space used by 
$V^{\prime \prime}$. The qubit in B is initialized to $|0\rangle$. $W$ behaves exactly in the same manner as $V^{\prime \prime}$ does, except that, in addition to all actions $V^{\prime \prime}$ would do, $W$ also sends $\mathrm{B}$ to the prover in the last message from the verifier and receives $\mathrm{B}$ from the prover in the last message from the prover. As for the final decision, $W$ accepts if and only if the content of $\mathrm{V}$ would make $V^{\prime \prime}$ accept and B contains 1 . Notice that $W$ accepts only if $V^{\prime \prime}$ would accept, and thus, the soundness is obviously at most $\frac{1}{2}+\frac{c-s}{4}$.

For perfect rewindability, we slightly modify the protocol of the honest prover in the case $x \in A_{\text {yes }}$. Given a protocol of the honest prover $P$ in the system with $V^{\prime \prime}$ and an initial state $\left|\psi_{\text {init }}\right\rangle$ in the system with $V^{\prime \prime}$ that achieves the maximal acceptance probability $p_{\max }$ when $V^{\prime \prime}$ communicating with this $P$, we construct a protocol of the honest prover $Q$ in the system with $W$ as follows. $Q$ uses $\left|\psi_{\text {init }}\right\rangle$ as the initial state and behaves exactly in the same manner as $P$ does, except that, upon receiving the last message from $W, Q$ applies to the qubit in $\mathrm{B}$ the one-qubit unitary transformation $U$ satisfying

$$
U:|0\rangle \mapsto \sqrt{1-\frac{1}{2 p_{\max }}}|0\rangle+\sqrt{\frac{1}{2 p_{\max }}}|1\rangle,
$$

in addition to all what the original $P$ would do. From the construction it is obvious that the maximum accepting probability of $W$ when communicating with $Q$ is exactly equal to $\frac{1}{2}$ and that this maximum is achieved when $Q$ uses $\left|\psi_{\text {init }}\right\rangle$ as the initial state. Finally, as the transformations of $V^{\prime \prime}$ are exactly implementable with the given finite-size gate set available for the verifier, so are the transformations of $W$.

Remark. In fact, in Lemma 32, it is sufficient for the claim that the functions $c$ and $s$ satisfy $c-s \geq 2^{-p}$ for some polynomially bounded function $p: \mathbb{Z}^{+} \rightarrow \mathbb{N}$.

\subsection{Proofs of Theorems 25 and 26}

Now we are ready to show Theorems 25 and 26. First we prove Theorem 26 , assuming that $m$ is an odd-valued function and $m \geq 3$. The case of general $m$ is proved in the same manner as this special case, except that the number of messages increases by one when $m(|x|)$ is even, which gives Theorem 25 .

Proof of Theorem 26. As $m$ is an odd-valued function and $m \geq 3$, there is a polynomially bounded function $r: \mathbb{Z}^{+} \rightarrow \mathbb{N}$ such that $m=2 r+1$. Let $A=\left(A_{\text {yes }}, A_{\text {no }}\right)$ be in $\operatorname{QIP}(2 r+1, c, s)$. Then from Lemma 32, $A$ has a perfectly rewindable $(2 r+1)$-message quantum interactive proof system with soundness $\frac{1}{2}-\frac{c-s}{4}$. Let $V$ be the verifier of this perfectly rewindable $(2 r+1)$-message quantum interactive proof system. We construct another $(2 r+1)$-message quantum verifier $W$ of a new quantum interactive proof system for $A$.

Fix an input $x$. Let $\mathrm{V}$ be the quantum register consisting of private qubits used by the original verifier $V$, and let $\mathrm{M}$ be the quantum register consisting of qubits used for communications in the original proof system. Let $V_{x, j}$ be the $j$ th transformation of $V$, for each $j \in\{1, \ldots, r(|x|)+1\}$, acting over $(\mathrm{V}, \mathrm{M})$. The new verifier $W$ uses the same registers $\mathrm{V}$ and $\mathrm{M}$ as the original verifier $V$. $W$ first receives the two registers $\mathrm{V}$ and $\mathrm{M}$, expecting that the state in $(\mathrm{V}, \mathrm{M})$ forms what $V$ would have after the last message from a prover had been received in the original proof system. $W$ then performs one of the two tests, called REFLECTION TEST and INVERTIBILITY TEST, chosen uniformly at random. In the REFLECTION TEST, $W$ first performs a phase-flip if the state in $(\mathrm{V}, \mathrm{M})$ would cause $V$ to accept when the last transformation $V_{x, r(|x|)+1}$ of $V$ was performed, and then moves to a backward simulation of the original system. $W$ accepts when the backward simulation does not produce a legal initial state of the original system. In the INVERTIBILITY TEST, $W$ just immediately moves to a backward simulation of the original system. This time, $W$ accepts when the backward simulation does produce a legal initial state of the original system. The exact protocol is described in Figure 8 Notice that the number of messages in this system is indeed $1+1+2(r(|x|)-1)+1=2 r(|x|)+1=m(|x|)$.

For the completeness, suppose that $x$ is in $A_{\text {yes }}$.

As the original system was perfectly rewindable, there exists a $(2 r+1)$-message quantum prover $P$ in the original system such that the maximum probability that $V$ accepts $x$ when communicating with this $P$ is exactly 


\section{Verifier's Protocol for Achieving Perfect Completeness (Odd-Number-Message Case)}

1. Receive quantum registers $V$ and $M$.

2. Choose $b \in\{0,1\}$ uniformly at random. If $b=0$, move to the REFLECTION TEST described in Step 3, while if $b=1$, move to the INVERTIBILITY TEST described in Step 4

\section{3. (REFLECTION TEST)}

3.1 Apply $V_{x, r(|x|)+1}$ to the state in $(\mathrm{V}, \mathrm{M})$. Perform a phase-flip (i.e., multiply -1 in phase) if the content of $(\mathrm{V}, \mathrm{M})$ corresponds to an accepting state of the original system. Apply $V_{x, r(|x|)+1}^{\dagger}$ to the state in $(\mathrm{V}, \mathrm{M})$, and send $\mathrm{M}$ to the prover.

3.2 For $j=r(|x|)$ down to 2 , do the following:

Receive $\mathrm{M}$ from the prover. Apply $V_{x, j}^{\dagger}$ to the state in $(\mathrm{V}, \mathrm{M})$, and send $\mathrm{M}$ to the prover.

3.3 Receive $\mathrm{M}$ from the prover. Apply $V_{x, 1}^{\dagger}$ to the state in $(\mathrm{V}, \mathrm{M})$. Reject if all the qubits in $\mathrm{V}$ are in state $|0\rangle$, and accept otherwise.

\section{4. (INVERTIBILITY TEST)}

4.1 Send $\mathrm{M}$ to the prover.

4.2 For $j=r(|x|)$ down to 2 , do the following:

Receive $\mathrm{M}$ from the prover. Apply $V_{x, j}^{\dagger}$ to the state in $(\mathrm{V}, \mathrm{M})$, and send $\mathrm{M}$ to the prover.

4.3 Receive $\mathrm{M}$ from the prover. Apply $V_{x, 1}^{\dagger}$ to the state in $(\mathrm{V}, \mathrm{M})$. Accept if all the qubits in $\mathrm{V}$ are in state $|0\rangle$, and reject otherwise.

Figure 8: Verifier's protocol for achieving perfect completeness with $m=2 r+1$.

$1 / 2$, where the maximum is taken over all possible initial states of $P$. Let $\mathrm{P}$ be the quantum register consisting of the private qubits of this $P$, and let $P_{x, j}$ be the $j$ th transformation of $P$, for each $j \in\{1, \ldots, r(|x|)+1\}$, acting over $(\mathrm{M}, \mathrm{P})$. Let $\left|\psi_{x}^{*}\right\rangle$ be an optimal initial state in (M,P) with which $P$ achieves the accepting probability $1 / 2$ (note that $P$ possesses the message register $\mathrm{M}$ at the beginning of the protocol, and that there always exists an optimal initial state that is pure).

Denote the Hilbert spaces associated with $\mathrm{V}, \mathrm{M}$, and $\mathrm{P}$ by $\mathcal{V}, \mathcal{M}$, and $\mathcal{P}$, respectively. Since the first action is done by $P$ in this original proof system, one can assume without loss of generality that $P_{x, 1}=I_{\mathcal{M} \otimes \mathcal{P}}$ (i.e., the first transformation of $P$ may be regarded as a part of preparing the initial state). Taking this into account, define the unitary transformation $Q_{x}$ acting over $(\mathrm{V}, \mathrm{M}, \mathrm{P})$ by

$$
Q_{x}=\left(V_{x, r(|x|)+1} \otimes I_{\mathcal{P}}\right)\left(I_{\mathcal{V}} \otimes P_{x, r(|x|)+1}\right) \cdots\left(V_{x, 2} \otimes I_{\mathcal{P}}\right)\left(I_{\mathcal{V}} \otimes P_{x, 2}\right)\left(V_{x, 1} \otimes I_{\mathcal{P}}\right)
$$

and further define the Hermitian matrix $M_{x}$ by

$$
M_{x}=\Pi_{\mathrm{init}} Q_{x}^{\dagger} \Pi_{\mathrm{acc}} Q_{x} \Pi_{\mathrm{init}},
$$

where $\Pi_{\text {init }}$ is the projection onto the subspace spanned by states in which all the qubits in $\vee$ are in state $|0\rangle$, and $\Pi_{\text {acc }}$ is that onto the subspace spanned by accepting states of the original system. Then the quantum state $\left|\phi_{x}^{*}\right\rangle$ in $(\mathrm{V}, \mathrm{M}, \mathrm{P})$ defined as $\left|\phi_{x}^{*}\right\rangle=|0\rangle_{\mathrm{V}} \otimes\left|\psi_{x}^{*}\right\rangle_{(\mathrm{M}, \mathrm{P})}$ is the eigenvector of $M_{x}$ with its corresponding eigenvalue $1 / 2$, 
since

$$
\max _{|\phi\rangle \in \mathcal{V} \otimes \mathcal{M} \otimes \mathcal{P}}\left\langle\phi\left|M_{x}\right| \phi\right\rangle=\max _{|\psi\rangle \in \mathcal{M} \otimes \mathcal{P}} \| \Pi_{\mathrm{acc}} Q_{x}(|0\rangle \otimes|\psi\rangle)\left\|^{2}=\right\| \Pi_{\mathrm{acc}} Q_{x}\left(|0\rangle \otimes\left|\psi_{x}^{*}\right\rangle\right) \|^{2}=\left\langle\phi_{x}^{*}\left|M_{x}\right| \phi_{x}^{*}\right\rangle=\frac{1}{2} .
$$

Now, with a $(2 r+1)$-message quantum prover $R$ in the constructed system who prepares the state $\left|\xi_{x}^{*}\right\rangle=\left(V_{x, r(|x|)+1}^{\dagger} \otimes I_{\mathcal{P}}\right) Q_{x}\left|\phi_{x}^{*}\right\rangle$ in $(\mathrm{V}, \mathrm{M}, \mathrm{P})$ as an initial state and applies $R_{x, 1}=I_{\mathcal{M} \otimes \mathcal{P}}$ and $R_{x, j}=P_{x, r(|x|)-j+3}$ for each $j \in\{2, \ldots, r(|x|)+1\}$, the constructed protocol may be viewed as performing the Modified REFLECTION PROCEDURE with its underlying quantum register $\mathrm{Q}=(\mathrm{V}, \mathrm{M}, \mathrm{P})$, unitary transformation

$$
U=\left(V_{x, r(|x|)+1}^{\dagger} \otimes I_{\mathcal{P}}\right) Q_{x}
$$

and projection operators

$$
\begin{aligned}
& \Delta_{0}=\Pi_{\text {init }}, \\
& \Pi_{0}=\left(V_{x, r(|x|)+1}^{\dagger} \otimes I_{\mathcal{P}}\right) \Pi_{\mathrm{acc}}\left(V_{x, r(|x|)+1} \otimes I_{\mathcal{P}}\right) .
\end{aligned}
$$

As the associated Hermitian operator

$$
M=\Delta_{0} U^{\dagger} \Pi_{0} U \Delta_{0}=\Pi_{\text {init }} Q_{x}^{\dagger} \Pi_{\text {acc }} Q_{x} \Pi_{\text {init }}=M_{x}
$$

has an eigenvalue $1 / 2$ with its corresponding eigenvector $\left|\phi_{x}^{*}\right\rangle=|0\rangle_{\vee} \otimes\left|\psi_{x}^{*}\right\rangle_{(\mathrm{M}, \mathrm{P})}$, from Proposition 29. the protocol results in acceptance with certainty with this prover $R$ and the initial state $\left|\xi_{x}^{*}\right\rangle=\left(V_{x, r(|x|)+1}^{\dagger} \otimes I_{\mathcal{P}}\right) Q_{x}\left|\phi_{x}^{*}\right\rangle=U\left|\phi_{x}^{*}\right\rangle$, which shows the perfect completeness.

Now for the soundness, suppose that $x$ is in $A_{\text {no. }}$.

Let $R$ be any $(2 r+1)$-message quantum prover of the constructed system, and let $\mathrm{R}$ be the quantum register consisting of the private qubits of $R$. Suppose that $R$ applies the unitary transformation $R_{x, j}$ to the state in $(\mathrm{M}, \mathrm{R})$ as the $j$ th transformation of $R$, for each $j \in\{1, \ldots, r(|x|)+1\}$.

Define the unitary transformation $Q_{x}$ acting over $(\mathrm{V}, \mathrm{M}, \mathrm{R})$ by

$$
Q_{x}=\left(V_{x, r(|x|)+1} \otimes I_{\mathcal{R}}\right)\left(I_{\mathcal{V}} \otimes R_{x, 2}^{\dagger}\right) \cdots\left(V_{x, 2} \otimes I_{\mathcal{R}}\right)\left(I_{\mathcal{V}} \otimes R_{x, r(|x|)+1}^{\dagger}\right)\left(V_{x, 1} \otimes I_{\mathcal{R}}\right),
$$

where $\mathcal{R}$ is the Hilbert space associated with the register $\mathrm{R}$. Then the constructed protocol may be viewed as performing the MODIFIED REFLECTION PROCEDURE with its underlying quantum register $Q=(V, M, R)$, unitary transformation

$$
U=\left(V_{x, r(|x|)+1}^{\dagger} \otimes I_{\mathcal{R}}\right) Q_{x}
$$

and projection operators

$$
\begin{aligned}
& \Delta_{0}=\Pi_{\text {init }}, \\
& \Pi_{0}=\left(V_{x, r(|x|)+1}^{\dagger} \otimes I_{\mathcal{R}}\right) \Pi_{\mathrm{acc}}\left(V_{x, r(|x|)+1} \otimes I_{\mathcal{R}}\right) .
\end{aligned}
$$

The associated Hermitian operator of this Modified REFLECTION PROCEDURE is given by

$$
M_{x}=\Delta_{0} U^{\dagger} \Pi_{0} U \Delta_{0}=\Pi_{\text {init }} Q_{x}^{\dagger} \Pi_{\text {acc }} Q_{x} \Pi_{\text {init }} .
$$

Consider the following $(2 r+1)$-message quantum prover $P^{\prime}$ in the original system: $P^{\prime}$ uses $\mathrm{R}$ as a register consisting of his/her private qubits, and applies $I_{\mathcal{M} \otimes \mathcal{R}}$ as his/her first transformation, and $R_{x, r(|x|)-j+3}^{\dagger}$ as his/her $j$ th transformation, for $j \in\{2, \ldots, r(|x|)+1\}$. Then, from the soundness property of the original system, no matter which state $P^{\prime}$ initially prepares, the accepting probability is at most $\frac{1}{2}-\frac{c(|x|)-s(|x|)}{4}$, which implies that all the 
eigenvalues of $M_{x}$ is at most $\frac{1}{2}-\frac{c(|x|)-s(|x|)}{4}$. Hence, from Proposition 30, the constructed protocol results in rejection with probability at least $\frac{(c(|x|)-s(|x|))^{2}}{16}$, which ensures the soundness $1-\frac{(c-s)^{2}}{16}$.

Finally, the protocol given in Figure 8 slightly deviates from the standard form of quantum interactive proof systems in that the length of the first message from a prover is different from the lengths of other messages, which may be easily modified into a standard-form system that has exactly the same number of messages and completeness and soundness parameters.

Now we prove Theorem 25. The proof is essentially the same as the proof of Theorem 26, and we analyze the case where the number of messages is even.

Proof of Theorem 25. Let $A=\left(A_{\text {yes }}, A_{\text {no }}\right)$ be in $\operatorname{QIP}(m, c, s)$. Then from Lemma 32, $A$ has a perfectly rewindable $m$-message quantum interactive proof system with soundness $\frac{1}{2}-\frac{c-s}{4}$. Let $V$ be the verifier of this perfectly rewindable $m$-message quantum interactive proof system. We construct an $(m+1)$-message quantum verifier $W$ of a new quantum interactive proof system for $A$. The construction is essentially the same as that in the proof of Theorem 26 .

Fix an input $x$. Suppose that $m(|x|) \geq 2$ is even, and write $m(|x|)=2 r(|x|)$ for some $r(|x|) \in \mathbb{N}$ (the proof of Theorem 26 already shows the case where $m(|x|)$ is odd). The exact protocol is described in Figure 9 , where the only difference from the protocol in Figure 8 lies in the condition of judging whether the state is initialized or not - now a state is a legal initial state only when all the qubits in both of $\mathrm{V}$ and $\mathrm{M}$ must be in state $|0\rangle$. Notice that the number of messages in this system is indeed $1+1+2(r(|x|)-1)+1=2 r(|x|)+1=m(|x|)+1$.

The analysis on this protocol is essentially the same as that in the proof of Theorem 26, and is omitted.

\subsection{Cases with Quantum Multi-Prover Interactive Proofs}

With essentially the same arguments discussed in this section, we can show similar properties even for quantum multi-prover interactive proof systems. The model of quantum multi-prover interactive proofs we use is that in the most general setting (i.e., both of a verifier and provers use quantum computation and communications, and provers can share arbitrary entanglement of arbitrarily large size). Let $\operatorname{QMIP}(k, m, c, s)$ be the class of problems having $m$-turn quantum $k$-prover interactive proof systems with completeness $c$ and soundness $s$. See Ref. [KKMV09] for rigorous definitions of the quantum multi-prover model and resulting complexity classes. Here we give only the statements of theorems, as proofs of those theorems are essentially same as Theorems 25 and 26 Note that these theorems give a more communication-efficient way of achieving perfect completeness in quantum multi-prover interactive proofs than the original method presented in Ref. [KKMV09], where the number of turns increases by a factor of three.

Theorem 33. For any polynomially bounded functions $k, m: \mathbb{Z}^{+} \rightarrow \mathbb{N}$ and polynomial-time computable functions $c, s: \mathbb{Z}^{+} \rightarrow[0,1]$ satisfying $m \geq 2$ and $c-s \geq 1 /$ for some polynomially bounded function $p: \mathbb{Z}^{+} \rightarrow \mathbb{N}$,

$$
\operatorname{QMIP}(k, m, c, s) \subseteq \operatorname{QMIP}\left(k, m+1,1,1-\frac{(c-s)^{2}}{16}\right) .
$$

Theorem 34. For any polynomially bounded function $k: \mathbb{Z}^{+} \rightarrow \mathbb{N}$, polynomially bounded odd-valued function $m: \mathbb{Z}^{+} \rightarrow 2 \mathbb{N}+1$, and polynomial-time computable functions $c, s: \mathbb{Z}^{+} \rightarrow[0,1]$ satisfying $m \geq 3$ and $c-s \geq 1 / p$ for some polynomially bounded function $p: \mathbb{Z}^{+} \rightarrow \mathbb{N}$,

$$
\operatorname{QMIP}(k, m, c, s) \subseteq \operatorname{QMIP}\left(k, m, 1,1-\frac{(c-s)^{2}}{16}\right) .
$$

Remark. Similar to the single-prover case, in fact, it is sufficient for the claims in Theorems 33 and 34 that the functions $c$ and $s$ satisfy $c-s \geq 2^{-p}$ for some polynomially bounded function $p: \mathbb{Z}^{+} \rightarrow \mathbb{N}$. 


\section{Verifier's Protocol for Achieving Perfect Completeness (Even-Number-Message Case)}

1. Receive quantum registers $V$ and $M$.

2. Choose $b \in\{0,1\}$ uniformly at random. If $b=0$, move to the REFLECTION TEST described in Step 3, while if $b=1$, move to the INVERTIBILITY TEST described in Step 4

\section{3. (REFLECTION TEST)}

3.1 Apply $V_{x, r(|x|)+1}$ to the state in $(\mathrm{V}, \mathrm{M})$. Perform a phase-flip (i.e., multiply -1 in phase) if the content of $(\mathrm{V}, \mathrm{M})$ corresponds to an accepting state of the original system. Apply $V_{x, r(|x|)+1}^{\dagger}$ to the state in $(\mathrm{V}, \mathrm{M})$, and send $\mathrm{M}$ to the prover.

3.2 For $j=r(|x|)$ down to 2 , do the following:

Receive $\mathrm{M}$ from the prover. Apply $V_{x, j}^{\dagger}$ to the state in $(\mathrm{V}, \mathrm{M})$, and send $\mathrm{M}$ to the prover.

3.3 Receive $\mathrm{M}$ from the prover. Apply $V_{x, 1}^{\dagger}$ to the state in $(\mathrm{V}, \mathrm{M})$. Reject if all the qubits in $(\mathrm{V}, \mathrm{M})$ are in state $|0\rangle$, and accept otherwise.

\section{4. (INVERTIBILITY TEST)}

4.1 Send $\mathrm{M}$ to the prover.

4.2 For $j=r(|x|)$ down to 2, do the following:

Receive $\mathrm{M}$ from the prover. Apply $V_{x, j}^{\dagger}$ to the state in $(\mathrm{V}, \mathrm{M})$, and send $\mathrm{M}$ to the prover.

4.3 Receive $\mathrm{M}$ from the prover. Apply $V_{x, 1}^{\dagger}$ to the state in $(\mathrm{V}, \mathrm{M})$. Accept if all the qubits in $(\mathrm{V}, \mathrm{M})$ are in state $|0\rangle$, and reject otherwise.

Figure 9: Verifier's protocol for achieving perfect completeness with $m(|x|)=2 r(|x|)$.

\section{Acknowledgements}

The authors are grateful to Richard Cleve for useful discussions, Attila Pereszlényi for pointing out an error in Lemma 23 in the earlier versions of this paper, and an anonymous reviewer for helpful comments on improving some parameters in the proof of Theorem 2 . This work is supported by the Grant-in-Aid for Scientific Research (A) No. 24240001 of the Japan Society for the Promotion of Science and the Grant-in-Aid for Scientific Research on Innovative Areas No. 24106009 of the Ministry of Education, Culture, Sports, Science and Technology in Japan. HK also acknowledges support from the Grant-in-Aid for Scientific Research (B) No. 21300002 of the Japan Society for the Promotion of Science. HN also acknowledges support from the Grant-in-Aids for Scientific Research (A) Nos. 21244007 and 23246071 of the Japan Society for the Promotion of Science and the Grant-in-Aid for Young Scientists (B) No. 22700014 of the Ministry of Education, Culture, Sports, Science and Technology in Japan.

\section{References}

[AALV09] Dorit Aharonov, Itai Arad, Zeph Landau, and Umesh Vazirani. The detectability lemma and quantum gap amplification [extended abstract]. In Proceedings of the 41st Annual ACM Symposium on Theory of Computing, pages 417-426, 2009. 
[AALV11] Dorit Aharonov, Itai Arad, Zeph Landau, and Umesh Vazirani. The 1D area law and the complexity of quantum states: A combinatorial approach. In 52nd Annual IEEE Symposium on Foundations of Computer Science, pages 324-333, 2011.

[Aar09] Scott Aaronson. On perfect completeness for QMA. Quantum Information and Computation, 9(12):0081-0089, 2009.

[AE11] Dorit Aharonov and Lior Eldar. On the complexity of commuting local Hamiltonians, and tight conditions for topological order in such systems. In 52nd Annual IEEE Symposium on Foundations of Computer Science, pages 334-343, 2011.

[Aha03] Dorit Aharonov. A simple proof that Toffoli and Hadamard are quantum universal. arXiv.org e-Print archive, arXiv:quant-ph/0301040, 2003.

[AKN98] Dorit Aharonov, Alexei Kitaev, and Noam Nisan. Quantum circuits with mixed states. In Proceedings of the Thirtieth Annual ACM Symposium on Theory of Computing, pages 20-30, 1998.

[ALM ${ }^{+}$98] Sanjeev Arora, Carsten Lund, Rajeev Motwani, Madhu Sudan, and Mario Szegedy. Proof verification and the hardness of approximation problems. Journal of the ACM, 45(3):501-555, 1998.

[AS98] Sanjeev Arora and Shmuel Safra. Probabilistic checking of proofs: A new characterization of NP. Journal of the ACM, 45(1):70-122, 1998.

[Bab85] László Babai. Trading group theory for randomness. In Proceedings of the Seventeenth Annual ACM Symposium on Theory of Computing, pages 421-429, 1985.

[Bra06] Sergey Bravyi. Efficient algorithm for a quantum analogue of 2-SAT. arXiv.org e-Print archive, arXiv:quant-ph/0602108, 2006.

[BSW11] Salman Beigi, Peter Shor, and John Watrous. Quantum interactive proofs with short messages. Theory of Computing, 7:101-117 (Article 7), 2011.

[Cho75] Man-Duen Choi. Completely positive linear maps on complex matrices. Linear Algebra and its Applications, 10(3):285-290, 1975.

[CKMR07] Matthias Christandl, Robert König, Graeme Mitchison, and Renato Renner. One-and-a-half quantum de Finetti theorems. Communications in Mathematical Physics, 273(2):473-498, 2007.

[ESY84] Shimon Even, Alan L. Selman, and Yacov Yacobi. The complexity of promise problems with applications to public-key cryptography. Information and Control, 61(2):159-173, 1984.

[Gro96] Lov K. Grover. A fast quantum mechanical algorithm for database search. In Proceedings of the Twenty-Eighth Annual ACM Symposium on the Theory of Computing, pages 212-219, 1996.

[GSU13] Sevag Gharibian, Jamie Sikora, and Sarvagya Upadhyay. QMA variants with polynomially many provers. Quantum Information and Computation, 13(1-2):0135-0157, 2013.

[Gut09] Gustav Gutoski. Quantum Strategies and Local Operations. PhD thesis, David R. Cheriton School of Computer Science, University of Waterloo, 2009. arXiv:1003.0038 [quant-ph].

[GZ11] Oded Goldreich and David Zuckerman. Another proof that BPP $\subseteq$ PH (and more). In Oded Goldreich, editor, Studies in Complexity and Cryptography, Miscellanea on the Interplay between Randomness and Computation, volume 6650 of Lecture Notes in Computer Science, pages 40-53. SpringerVerlag, 2011. Electronic Colloquium on Computational Complexity, Report TR97-045, 1997. 
[Jam72] Andrzej Jamiołkowski. Linear transformations which preserve trace and positive semidefiniteness of operators. Reports on Mathematical Physics, 3(4):275-278, 1972.

[JJUW11] Rahul Jain, Zhengfeng Ji, Sarvagya Upadhyay, and John Watrous. QIP $=$ PSPACE. Journal of the ACM, 58(6):Article 30, 2011.

[JKNN12] Stephen P. Jordan, Hirotada Kobayashi, Daniel Nagaj, and Harumichi Nishimura. Achieving perfect completeness in classical-witness quantum Merlin-Arthur proof systems. Quantum Information and Computation, 12(5-6):0461-0471, 2012.

[Kit99] Alexei Yu. Kitaev. Quantum NP. Talk at the 2nd Workshop on Algorithms in Quantum Information Processing, January 1999.

[KKMV09] Julia Kempe, Hirotada Kobayashi, Keiji Matsumoto, and Thomas Vidick. Using entanglement in quantum multi-prover interactive proofs. Computational Complexity, 18(2):273-307, 2009.

[Kni96] Emanuel Knill. Quantum randomness and nondeterminism. Technical Report LAUR-96-2186, Los Alamos National Laboratory, 1996. arXiv.org e-Print archive, arXiv:quant-ph/9610012.

[KR05] Robert König and Renato Renner. A de Finetti representation for finite symmetric quantum states. Journal of Mathematical Physics, 46(12):122108, 2005.

[KSV02] Alexei Yu. Kitaev, Alexander H. Shen, and Mikhail N. Vyalyi. Classical and Quantum Computation, volume 47 of Graduate Studies in Mathematics. American Mathematical Society, 2002.

[Kup09] Greg Kuperberg. How hard is it to approximate the Jones polynomial? arXiv.org e-print archive, arXiv:0908.0512 [quant-ph], 2009.

[KW00] Alexei Kitaev and John Watrous. Parallelization, amplification, and exponential time simulation of quantum interactive proof systems. In Proceedings of the Thirty-Second Annual ACM Symposium on Theory of Computing, pages 608-617, 2000.

[MW05] Chris Marriott and John Watrous. Quantum Arthur-Merlin games. Computational Complexity, 14(2):122-152, 2005.

[NC00] Michael A. Nielsen and Isaac L. Chuang. Quantum Computation and Quantum Information. Cambridge University Press, 2000.

[NS03] Ashwin Nayak and Peter Shor. Bit-commitment-based quantum coin flipping. Physical Review A, 67(1):012304, 2003.

[NWZ09] Daniel Nagaj, Pawel Wocjan, and Yong Zhang. Fast amplification of QMA. Quantum Information and Computation, 9(11-12):1053-1068, 2009.

[Shi02] Yaoyun Shi. Both Toffoli and Controlled-NOT need little help to do universal quantum computing. Quantum Information and Computation, 3(1):084-092, 2002.

[Sho96] Peter W. Shor. Fault-tolerant quantum computation. In 37th Annual Symposium on Foundations of Computer Science, pages 56-65, 1996.

[SR02] Robert W. Spekkens and Terry Rudolph. Degrees of concealment and bindingness in quantum bit commitment protocols. Physical Review A, 65(1):012310, 2002. 
[Vya03] Mikhail N. Vyalyi. QMA = PP implies that PP contains PH. Electronic Colloquium on Computational Complexity, Report TR03-021, 2003.

[Wat00] John Watrous. Succinct quantum proofs for properties of finite groups. In 41st Annual Symposium on Foundations of Computer Science, pages 537-546, 2000.

[Wat03] John Watrous. PSPACE has constant-round quantum interactive proof systems. Theoretical Computer Science, 292(3):575-588, 2003.

[Wat09a] John Watrous. Quantum computational complexity. In Robert A. Meyers, editor, Encyclopedia of Complexity and Systems Science, pages 7174-7201. Springer-Verlag, 2009.

[Wat09b] John Watrous. Zero-knowledge against quantum attacks. SIAM Journal on Computing, 39(1):25-58, 2009.

[ZF87] Stathis Zachos and Martin Fürer. Probabilistic quantifiers vs. distrustful adversaries. In Foundations of Software Technology and Theoretical Computer Science, Seventh Conference, volume 287 of Lecture Notes in Computer Science, pages 443-455, 1987. 